\title{
Measuring gravitational waves from binary black hole coalescences. I. Signal to noise for inspiral, merger, and ringdown
}

\author{
Éanna É. Flanagan \\ Cornell University, Newman Laboratory, Ithaca, New York 14853-5001
}

Scott A. Hughes

Theoretical Astrophysics, California Institute of Technology, Pasadena, California 91125

(Received 17 January 1997; published 2 April 1998)

\begin{abstract}
We estimate the expected signal-to-noise ratios (SNRs) from the three phases (inspiral, merger, and ringdown) of coalescing binary black holes (BBHs) for initial and advanced ground-based interferometers (LIGOVIRGO) and for the space-based interferometer LISA. Ground-based interferometers can do moderate SNR (a few tens), moderate accuracy studies of BBH coalescences in the mass range of a few to about 2000 solar masses; LISA can do high SNR (of order $10^{4}$ ), high accuracy studies in the mass range of about $10^{5}-10^{8}$ solar masses. BBHs might well be the first sources detected by LIGO-VIRGO: they are visible to much larger distances - up to $500 \mathrm{Mpc}$ by initial interferometers - than coalescing neutron star binaries (heretofore regarded as the "bread and butter" workhorse source for LIGO-VIRGO, visible to about $30 \mathrm{Mpc}$ by initial interferometers). Low-mass BBHs (up to $50 M_{\odot}$ for initial LIGO interferometers, $100 M_{\odot}$ for advanced, $10^{6} M_{\odot}$ for LISA) are best searched for via their well-understood inspiral waves; higher mass BBHs must be searched for via their poorly understood merger waves and/or their well-understood ringdown waves. A matched filtering search for massive BBHs based on ringdown waves should be capable of finding BBHs in the mass range of about $100 M_{\odot}-700 M_{\odot}$ out to $\sim 200 \mathrm{Mpc}$ for initial LIGO interferometers, and in the mass range of $\sim 200 M_{\odot}$ to $\sim 3000 M_{\odot}$ out to about $z=1$ for advanced interferometers. The required number of templates is of the order of 6000 or less. Searches based on merger waves could increase the number of detected massive BBHs by a factor of the order of 10 over those found from inspiral and ringdown waves, without detailed knowledge of the waveform shapes, using a noise monitoring search algorithm which we describe. A full set of merger templates from numerical relativity simulations could further increase the number of detected BBHs by an additional factor of up to 4. [S0556-2821(98)06508-4]
\end{abstract}

PACS number(s): 04.80.Nn, 04.25.Dm, 04.30.Db, 95.55.Ym

\section{INTRODUCTION AND SUMMARY}

\section{A. Coalescences of black hole binaries}

It has long been recognized that coalescences of binary black hole (BBH) systems could be an important source of gravitational waves $[1,2]$, both for ground-based interferometric detectors such as the Laser Interferometric Gravitational Wave Observatory (LIGO) [3] and VIRGO [4] currently under construction, and also for the possible future space-based Laser Interferometer Space Antenna (LISA) [57]. The orbits of BBHs gradually decay from energy and angular momentum loss to gravitational radiation. Eventually, they merge to form a single black hole.

The process of coalescence can be divided into three more or less distinct phases:

An adiabatic inspiral, during which the gravitational radiation reaction time scale is much longer than the orbital period. The inspiral ends when the binary orbit becomes relativistically dynamically unstable at an orbital separation of $r \sim 6 M$ (in units where $G=c=1$ ) [8,9]. The gravitational waves from the inspiral carry encoded within them the masses and spins of the two black holes, some of the binary's orbital elements, and the distance to the binary $[1,10]$.

Towards the end of inspiral, the black holes encounter a dynamical instability and make a gradual transition from a radiation-reaction driven inspiral to a freely falling plunge $[8,11,12]$. After the plunge, the black holes would still merge even if the radiation reaction could be turned off. We will call the subsequent plunge and violent collision the merger phase. Gravitational waves from the merger could be rich with information about the dynamics of relativistic gravity in a highly nonlinear, highly dynamic regime which is poorly understood today.

As the system settles down to a stationary Kerr state, the nonlinear dynamics of the merger gradually becomes more and more describable as oscillations of the final black hole's quasinormal modes $[13,14]$. The corresponding gravitational waves consist of a superposition of exponentially damped sinusoids. We will call the phase of the coalescence for which the gravitational waves are dominated by the strongest $l=m=2$ quasinormal mode the ringdown. The ringdown waves carry information about the mass and spin of the final black hole $[15,16]$. (For want of a better terminology, we will always use coalescence to refer to the entire process of inspiral, merger and ringdown, and reserve the word merger for the phase intermediate between inspiral and ringdown.)

In this paper we focus primarily on BBHs in which the masses of the two black holes are approximately the same, although we do also consider sources with one black hole much smaller than the other. We consider three different classes of BBHs:

(i) Solar mass black hole binaries: these are binaries that are formed either from massive main-sequence progenitor binary stellar systems (field binaries) or from capture pro- 
cesses in globular clusters or galactic centers (capture binaries). Field binaries are expected to have total masses in the range $10 M_{\odot} \lesssim M \lessgtr 50 M_{\odot}$, but not much larger than this, while capture binaries could have somewhat larger masses [17]. The event rate of solar-mass BBH coalescences is not well known. For globular cluster capture binaries, Sigurdsson and Hernquist argue that generically at least one BBH coalescence should occur per core-collapsed globular cluster [18], yielding $\sim 3 \mathrm{yr}^{-1}$ in a distance of $600 \mathrm{Mpc}$ using the extrapolation method of Sec. 3.1 of Ref. [19]. This rate is one or two orders of magnitude smaller than the expected event rate for what has traditionally been regarded as the most promising source for ground-based interferometers, coalescences of neutron-star-neutron-star (NS-NS) binaries $[3,10]$ (about $10^{-5} \mathrm{yr}^{-1}$ in our Galaxy, or several per year in a distance of $200 \mathrm{Mpc}$ [20,19,21-23]). However, BBH systems can be seen to much greater distances than NS-NS systems, and so it is possible that BBH coalescences will be seen before NS-NS coalescences. For field binaries, estimates of the coalescence rate by experts in binary evolution theory range from $\sim 10^{-8} \mathrm{yr}^{-1}$ to $\sim 10^{-6} \mathrm{yr}^{-1}$ in our Galaxy [22,24], to completely negligible [25]. There are large uncertainties associated with these theoretical estimates of the coalescence rates [26].

(ii) Intermediate mass black hole binaries: these are binaries with total masses in the range $50 M_{\odot} \lesssim M \lesssim(\mathrm{a}$ few) $\times 10^{3} M_{\odot}$. In contrast to the cases of solar mass black holes and supermassive black holes (discussed below), there is little direct observational evidence for the existence of black holes in this mass range [27]. Despite the lack of evidence, it is plausible that black holes in this mass range are formed in the cores of globular clusters or in galactic nuclei in the process of formation of a supermassive black hole [30]. Simulations by Quinlan and Shapiro suggest that black holes with $M \sim 100 M_{\odot}$ to $1000 M_{\odot}$ could be formed in the evolution of dense stellar clusters of main sequence stars in galactic nuclei [17], and that coalescences of binaries of such black holes could be possible en route to the formation of a supermassive black hole. Even if the coalescence rate of intermediate mass BBHs is only $10^{-4}$ that of NS-NS binaries, they are visible to such great distances that they would still be seen more often than NS-NS binaries by initial and advanced LIGO interferometers, and thus could be the first detected type of source. (See Sec. I E for further details.)

(iii) Supermassive black hole binaries: there is a variety of strong circumstantial evidence that supermassive black holes (SMBHs) in the mass range $10^{6} M_{\odot}$ to $10^{9} M_{\odot}$ are present in quasars and active galactic nuclei, and that a large fraction of nearby massive spiral and elliptical galaxies harbor quiescent SMBHs $[7,31,30]$. One of the main goals of the LISA project is to detect and monitor various processes involving SMBHs, such as the capture of compact stars $[2,7,10,32,33]$ and their formation $[2,7]$. In particular, the coalescences of SMBH binaries that are formed in galaxy mergers, in which the individual SMBHs are driven together by dynamical friction and gas accretion until a gravitational radiation reaction takes over [34], have often been suggested as a promising source for space-based interferometers $[1,2,7,10,35,36]$. Such coalescences would be detectable throughout the observable universe with large signal to noise ratios $[7,10]$. There is some observational evidence for SMBH binaries: wiggles in the radio jet of QSO $1928+738$ have been attributed to the orbital motion of a SMBH binary [37], as have time variations in quasar luminosities [38] and in emission line redshifts [39]. The overall event rate is uncertain, but could be large $(\gtrsim 1 / \mathrm{yr})$, especially if the hierarchical scenario for structure formation is correct [36].

\section{B. Status of theoretical calculations of the gravitational-wave signal}

Detailed theoretical understanding and predictions of the gravitational waveforms $h_{+}(t)$ and $h_{\times}(t)$ produced in BBH coalescences will facilitate both the detection of the gravitational-wave signal and the extraction of its information. In situations where a complete family of theoretical template waveforms is available, it will be possible to use Wiener optimal filtering ("matched filtering") to search the interferometer data streams and to detect the signal $[1,40]$. The resulting signal-to-noise ratios (SNRs) can be larger than those obtainable without theoretical templates by a substantial factor; see Sec. II. Thus, while it is possible to detect the various phases of BBH coalescences without theoretical templates, such templates can greatly increase the effective range of the interferometers and the event detection rate. Such theoretical template waveforms are available for the inspiral and ringdown phases of the coalescence, but not yet for the merger phase, as we now discuss.

For the inspiral, the gravitational waves and orbital evolution can be described reasonably well using the postNewtonian approximation to general relativity. To date, inspiral waveforms have been calculated to post-2.5Newtonian order [41], and the prospects look good for obtaining waveforms up to post-3.5-Newtonian order $[42,43]$. Post-Newtonian templates will be fairly accurate over most of the inspiral, the most important error being a cumulative phase lag $[44,45]$. This cumulative phase lag will not be important for searches for inspiral waves; the template phasing error will be largely compensated for by systematic errors in best-fit values of the binary's parameters, and the signals will still be found [44,46-48]. By contrast, template inaccuracies will be significant when one attempts to extract from the data the binary's parameters. In particular, postNewtonian templates' errors start to become very significant around an orbital separation of $r \sim 12 M$ [49], well before the end of the inspiral at the dynamical orbital instability ( $r$ $\sim 6 M)$. Templates for the phase of the inspiral between roughly $12 M$ and $6 M$ will most likely have to be calculated using methods other than the post-Newtonian approximation. The methods of full blown numerical relativity cannot be applied to this "intermediate binary black hole" (IBBH) phase, since the total time taken to evolve from $12 M$ to $6 M$ is about $1500 M$, too long for supercomputer simulations to evolve. Analytical and numerical methods for calculating IBBH waveforms based on the adiabatic approximation are under development [50]; it is likely they will be successfully implemented before gravitational-wave interferometers begin measurements [51].

Waveforms from the dynamic, complicated merger can only be obtained from numerical relativity. Unlike mergers of neutron star binaries, BBH mergers are particularly clean in the sense that there is no microphysics or hydrodynamics 
to complicate simulations of the evolution, and external perturbations are negligible: the entire merger can be described as a solution to the vacuum Einstein equation [53]. Finding that solution is not a particularly easy task: a major computational effort to evolve the vacuum Einstein equation for BBH mergers using massive computational resources is currently underway, funded by the National Science Foundation's Grand Challenge program $[54,55]$.

The ringdown phase of the coalescence can be accurately described using perturbation theory on the Kerr spacetime background [56]. The gravitational waveforms from this phase are well understood, being just exponentially damped sinusoids. Thus, matched filtering is feasible for searches for ringdown waves.

\section{Purpose of this paper}

The principal purpose of this paper is to estimate, in more detail than has been done previously, the prospects for measuring gravitational waves from the three different phases of coalescence events, for various different detectors, and for a wide range of $\mathrm{BBH}$ masses. We estimate in each case the distances to which the different types of source can be seen by calculating expected SNRs. In particular, we determine for each $\mathrm{BBH}$ mass and each detector whether a coalescence event is most effectively detected by searching for the inspiral, or the merger, or the ringdown. We also determine how much the availability of theoretical templates for the merger could increase the event detection rate. Previous estimates of SNRs for ground-based interferometers have focused on the inspiral $[1,44]$ and ringdown $[15,16]$, and also focused on solar-mass BBHs. For space-based interferometers, previous estimates of SNRs from the merger $[7,10]$ were restricted to specific masses and did not consider the ringdown.

In a companion paper, we discuss in detail the useful information carried by the three phases of the gravitationalwave signal, and methods and prospects for extracting this information both with and without templates for the merger phase [57].

\section{Estimating the signal-to-noise ratios: Method and assumptions}

We calculate SNRs for three different types of interferometer: initial and advanced ground-based interferometers (LIGO-VIRGO), and the proposed space-based interferometer LISA. The noise spectra of the initial and advanced ground-based interferometers we took from Ref. [3] and that for LISA from Ref. [7]. Our approximate versions of these noise spectra are given in Eqs. (4.1)-(4.4), and are illustrated in Figs. $1-3$ in Sec. V A.

We consider the following three different signal-detection methods:

(i) Matched filtering searches: For those phases of the coalescence for which a complete set of theoretical templates will be available (the inspiral, the ringdown, and possibly the merger), matched filtering can be used to search for the waves $[1,40,58-60]$. For any source of waves, the SNR $\rho$ obtained from matched filtering is related to the gravitational waveform $h(t)$ measured by the interferometer and to the spectral density $S_{h}(f)$ of the strain noise in the interferometer via [61]

$$
\rho^{2}=4 \int_{0}^{\infty} \frac{|\widetilde{h}(f)|^{2}}{S_{h}(f)} d f
$$

where $\widetilde{h}(f)$ is the Fourier transform of $h(t)$ defined by Eq. (2.3). The SNR (1.1) depends, through the waveform $h(t)$, on the orientation and position of the source relative to the interferometer. In Sec. II C we show that if we perform an rms average over source orientations and positions (at a fixed distance), the rms SNR thus obtained depends only on the energy spectrum $d E / d f$ of the emitted gravitational waves. The resulting relationship between the waves' energy spectrum and the rms angle-averaged SNR forms the basis for most of our calculations. It is given by [cf. Eq. (2.30)]

$$
\left\langle\rho^{2}\right\rangle=\frac{2(1+z)^{2}}{5 \pi^{2} D(z)^{2}} \int_{0}^{\infty} d f \frac{1}{f^{2} S_{h}(f)} \frac{d E}{d f}[(1+z) f],
$$

where $z$ is the source's cosmological redshift and $D(z)$ its luminosity distance. In order for a signal to be detected, the waves' measured SNR must be larger than a certain threshold which we discuss in Sec. II C [cf. Eq. (2.9)].

(ii) Band-pass filtering searches: For the merger phase, a complete set of theoretical templates may not be available, and so methods other than matched filtering will need to be used. Band-pass filtering, followed by setting a detection threshold in the time domain, is a simple method of searching an interferometer data stream for bursts of unknown form [40]. In Sec. II A we derive an approximate relation between the SNR obtainable from band-pass filtering, and the SNR (1.1) obtainable from matched filtering, for any burst of waves:

$$
\left(\frac{S}{N}\right)_{\text {band-pass }} \approx \frac{1}{\sqrt{2 T \Delta f}}\left(\frac{S}{N}\right)_{\text {matched }} .
$$

Here $T$ is the duration of the burst and $\Delta f$ is the bandwidth of the band-pass filter [cf. Eq. (2.15)]. The quantity $2 T \Delta f$ is the dimension of the linear space of signals being searched for, and is roughly the same as the "number of cycles" of the gravitational waveform. In Sec. VI B, we use the formula (1.3) to estimate the SNRs from band-pass filter searches for merger waves, by inserting on the right hand side the rms angle-averaged matched-filter SNR (1.2) and by making estimates of $T$ and $\Delta f$.

(iii) Noise-monitoring, nonlinear filtering searches: The traditional view has been that the SNR (1.3) is about the best that can be achieved in the absence of templates, that is, that the gain in SNR obtainable from matched filtering is approximately the square root of the number of cycles in the gravitational wave signal [Eq. (2.15) below]. This view is based on the assumption that the search method used in the absence of templates is band-pass filtering or something very similar. However, we suggest in Sec. II B an alternative search method, motivated by Bayesian analyses and incorporating nonlinear filtering, which performs much better than band-pass filtering and in some cases almost as well as matched filtering. In essence, one monitors the noise level in 
the data stream in a certain frequency band, over short time scales, and looks for statistically significant changes. The noise level is estimated by calculating the quantity

$$
\frac{1}{T} \int_{-T / 2}^{T / 2} d \tau s(t+\tau)^{2}
$$

where $T$ is the maximum expected duration of the signal, and $s(t)$ is a suitably pre-filtered version of the data stream.

The efficiency of this noise-monitoring search method cannot usefully be described in terms of an SNR, since the detection statistic is non-Gaussian. Instead, its efficiency can be described in the following way. Let $\rho$ denote the SNR that would be obtained if matched filtering were possible [Eq. (1.1)]. We use $\rho$ as a convenient parameterization of the signal strength; as such, it is meaningful even in situations where matched filtering cannot be carried out. A signal will be detected with high confidence using the noise-monitoring technique whenever $\rho$ is larger than a threshold $\rho_{*}$ given by Eq. (2.29) below. In practice, $\rho_{*}$ is slightly larger than the threshold for matched filtering but not greatly larger.

The relation (1.2) forms the basis of our SNR calculations. We use the thresholds (2.9) and (2.29) to deduce from the SNR values the detectability of the various parts of the gravitational wave signal. To calculate the SNRs, we also need to specify the waves' energy spectra for the three different phases of the coalescence. As we now outline, the waves' energy spectrum is essentially known for the inspiral and ringdown phases, and we make an educated guess for the merger. Section III gives more details.

Inspiral energy spectrum: We use the leading order expression for $d E / d f$ obtained using Newtonian gravity supplemented by the quadrupole formula [62] [Eq. (3.14)]. Strictly speaking, this spectrum describes the SNR that would be achieved by searching for Newtonian, quadrupole waves using Newtonian, quadrupole templates. The actual SNR obtained when searching for a real, general-relativistic inspiral using post-Newtonian templates should deviate from this by only a few tens of a percent [63]. We terminate the spectrum at the frequency $f_{\text {merge }}=0.02 / M$ which is (roughly) the frequency of quadrupole waves emitted at the orbital dynamical instability at $r \sim 6 M$ [8]. For LISA, we assume that the measurement process lasts at most $1 \mathrm{yr}$, and choose the frequency at which the spectrum starts accordingly.

Ringdown energy spectrum: The spectrum that we use [Eq. (3.19)] is determined, up to its overall amplitude, by the properties of the $l=m=2$ quasi-normal ringing (QNR) mode of the final Kerr black hole. This mode is the most slowly damped of all QNR modes, and so we expect it to dominate the last stages of gravitational-wave emission. The QNR spectrum depends on three parameters: the modes' frequency $f_{\text {qnr }}$, damping time $\tau$, and initial amplitude of excitation, which in turn depend on the mass $M$ and dimensionless spin parameter $a$ of the final black hole and on the total energy radiated in the ringdown. The spectrum is peaked at $f=f_{\mathrm{qnr}}$ with width $\Delta f \sim 1 / \tau$.

We (somewhat arbitrarily) assume $a=0.98$. It seems likely that in many coalescences the spin of the final black hole will be close to maximal, since the total angular momentum of the binary at the end of the inspiral is $\sim 0.9 \mathrm{M}^{2}$ when the individual black holes are non-spinning [65], and can be larger when they are spinning. Exactly how close to extremal the final black hole will be is a matter that probably will not be decided until supercomputer simulations-or observations - settle the issue. In any case, the ringdown SNR values that we obtain depend only weakly on our assumed value of $a$ [cf. Eq. (B14)], for fixed total energy radiated in the ringdown.

The overall amplitude of the ringdown signal depends upon one's delineation of where "merger" ends and "ringdown" begins, which is somewhat arbitrary. For equal-mass BBHs, we assume a value of the overall amplitude that corresponds to a total radiated energy in the ringdown of $0.03 M$, i.e., a $3 \%$ radiation efficiency. This number is based on a quadrupole-formula-based estimate of the QNR amplitude when the distortion of the horizon of the black hole is of order unity (cf. Sec. III D). Although this radiation efficiency may seem rather high, there have been numerical evolutions of distorted, spinning black holes in which the ringdown waves carry away $\geq 3 \%$ of the black hole's total mass [66].

For non-equal-mass black holes, we assume that the total energy radiated in the ringdown is $F(\mu / M) 0.03 M$, where

$$
F(\mu / M)=(4 \mu / M)^{2}
$$

and $\mu$ is the reduced mass of the binary. The reduction factor (1.5) gives the correct results for equal masses and also gives the correct scaling law in the regime $\mu \ll M$. For general mass ratios, it is probably a good approximation.

Merger energy spectrum: Realistic merger energy spectra will vary substantially from event to event (depending on the initial BH spins). Currently, we have very little concrete information about such spectra, pending supercomputer simulations. We adopt the following crude model for equal-mass BBHs [Eq. (3.13) below]: a flat spectrum $d E / d f=$ const extending from the frequency $f_{\text {merge }}=0.02 / M$ of quadrupole waves at the end of inspiral to the quasinormal ringing frequency $f_{\text {qnr }}=0.13 / M$, with amplitude such that the total radiated energy in the merger is $10 \%$ of the total mass energy of the spacetime. In Sec. III B we describe various circumstantial pieces of evidence, culled from the literature, which motivated this choice of energy spectrum. In particular, we outline two different "handwaving" arguments which suggest that in favorable cases the merger radiation efficiency may be as high as our assumed value of $\sim 10 \%$. One of these arguments, due originally to Smarr [67] and explored by Detweiler [68], is based on extrapolation of perturbation theory results; the other argument is based on angular momentum conservation.

Our assumed radiation efficiencies of 3\% and $10 \%$ for the ringdown and merger phases should be interpreted as reasonable upper bounds that could be achieved in favorable cases, rather than as best-guess estimates. We note that numerical simulations that have been performed to date (which are restricted to axisymmetric situations) generally yield lower radiation efficiencies than we have assumed [69]; moreover, these axisymmetric simulations generally find that ringdown waves carry most of the radiated energy. In Sec. III B we argue that the radiated energy in the merger phase could be boosted by the lack of symmetry in generic black hole mergers and especially by the individual black holes' spins (if these spins are large). 
For non-equal-mass BBHs, we again reduce the energy spectrum by the factor (1.5), while the upper and lower frequencies $f_{\text {merge }}$ and $f_{\text {qnr }}$ are taken to be independent of $\mu$.

\section{E. Signal-to-noise ratios: Results and implications}

By inserting our assumed energy spectra (3.14), (3.13) and (3.18) into Eq. (1.2), we obtain matched-filtering SNRs for the three different phases of BBH coalescences as a function of the redshifted total mass $(1+z) M$ of the binary. The results are summarized in Appendix B, and graphed in Figs. 4, 5 and 6. In Sec. III E we estimate that the number of independent frequency bins $\mathcal{N}_{\text {bins }} \equiv 2 T \Delta f$ characterizing the merger falls in the range $10 \leq \mathcal{N}_{\text {bins }} \leqslant 30$; a conservative upper bound is $\sim 60$. We use this upper bound in Sec. VI B to estimate the SNR threshold (2.29) for merger waves using noise-monitoring searches when templates are unavailable. We discuss the implications of these SNRs and SNR thresholds in Sec. VI; here we summarize our main conclusions:

Ground-based interferometers can study black-hole mergers in the mass range (a few) $M_{\odot}$ to $\sim 2000 M_{\odot}$; LISA, by contrast, can study mergers in the mass range $10^{5} M_{\odot} \lesssim(1$ $+z) M \lesssim 10^{8} M_{\odot}$.

Ground-based interferometers can do moderate SNR (a few tens), moderate accuracy studies of the dynamics of merging black holes. LISA, by contrast, can do high SNR (a few $\times 10^{4}$ ), high-accuracy studies.

Coalescing black holes may well be the first sources detected by LIGO-VIRGO: because of their larger masses, they can be seen to much greater distances than coalescing neutron star binaries. (With the initial LIGO interferometers, BBHs with $M \lessgtr 50 M_{\odot}$ can be seen to $\sim 250 \mathrm{Mpc}$, whereas binary neutron stars can be seen to $\sim 25 \mathrm{Mpc}$ [70]). The distance gain for BBHs could easily compensate for their smaller birth rate discussed above.

Low-mass BBHs $\left[M \leqslant 30 M_{\odot}\right.$ for initial LIGO interferometers, $M \leqslant 80 M_{\odot}$ for advanced, $(1+z) M \leqslant 3 \times 10^{6} M_{\odot}$ for LISA] are best searched for via their well-understood inspiral waves; more massive BBHs must be searched for via their poorly understood merger waves and/or their wellunderstood ringdown waves.

A search for massive BBHs based on the ringdown waves can be performed using matched filtering. We show in Sec. VI A that the number of templates needed for such a search is about 6000 or less, assuming that one wants the event rate reduction due to discreteness of the template family to be no more than $10 \%$. Such a search with the first LIGO interferometers should be capable of finding equal-mass BBHs in the mass range $100 M_{\odot}-700 M_{\odot}$ out to about $200 \mathrm{Mpc}$. With advanced LIGO interferometers, BBHs with $200 M_{\odot} \leq M(1$ $+z) \leqslant 3000 M_{\odot}$ should be detectable out to $z \sim 1$, and with LISA, BBHs with $10^{6} M_{\odot} \lesssim(1+z) M \lessgtr 3 \times 10^{8} M_{\odot}$ should be visible out to $z \gtrsim 100$. These distances are reduced by a factor of $\sim(4 \mu / M)$ for non-equal-mass BBHs.

The effectiveness of a search based on the merger waves will depend on how much one has learned about the waves from numerical relativity. With only knowledge of the merger waves' range of frequency bands and range of temporal durations, a search can be performed using the noisemonitoring search algorithm discussed above. Such a search could increase the number of discovered BBHs by a factor of $\sim 10$ over those found from the inspiral and ringdown waves. A full set of merger templates based on numerical relativity simulations could further increase the event rate by an additional factor of up to $\sim 4$.

\section{F. Organization of this paper}

The body of the paper is organized as follows. In Sec. II we discuss the three methods of searching for gravitational wave signals referred to above. In Sec. III we discuss our assumptions about the BBH gravitational-wave signal: the splitting into three epochs, details of the emitted gravitational-wave energy spectrum during each epoch, and reasonable estimates of the duration and bandwidth of the dynamical merger. In Sec. IV we devise a simple piece-wise power-law analytic fit [Eq. (4.1)] to the noise spectra of an initial LIGO interferometer, an advanced LIGO interferometer, and a space-based LISA interferometer. This single formula, by adjustment of its parameters, can describe all three interferometer types.

In Sec. V we insert these noise spectra models and the gravitational-wave energy spectra into the general SNR formula (1.2) to produce the matched filtering SNR for each type of interferometer and for each phase of BBH coalescence. Detailed SNR results are given in Appendix B. We give intuitive insight into these SNRs in Sec. V A by reexpressing the power SNR for a source as

$$
\rho^{2}=\int d(\ln f)\left[h_{\text {char }}(f) / h_{n}(f)\right]^{2},
$$

where $h_{\text {char }}(f)$ is the source's "characteristic amplitude" as a function of frequency, and $h_{n}(f)$ is the detector's rms noise in a bandwidth equal to frequency for sources with random orientations. We give plots of $h_{\text {char }}(f)$ and $h_{n}(f)$ for five specific examples of binaries with widely varying masses and distances. In Sec. V B, we plot and discuss the SNRs as functions of source mass [Figs. 4, 5, and 6]. These plots are the foundation for our conclusions, summarized above, about what features of which binaries should be observable with which interferometers. A detailed discussion of these conclusions is given in Sec. VI. In Sec. VI A, we estimate the number of templates required for a search for ringdown waves based on matched filtering and estimate the SNR detection thresholds and, hence, the range of the various interferometers for ringdown waves. In Sec. VI B we examine the prospects for searches for $\mathrm{BBHs}$ via their merger waves, both with and without templates.

\section{DERIVATION OF GENERAL FORMULAS FOR SIGNAL-TO-NOISE RATIOS AND DETECTION THRESHOLDS}

In this section we discuss the various signal-search methods which were briefly described in the Introduction. In Sec. II A we derive the approximate relation (1.3) between the SNR achievable using matched filtering searches for signals and the SNR obtainable via band-pass filtering searches. In Sec. II B we describe our proposed noise-monitoring search method, and derive the detection threshold (2.29) discussed in Sec. I E. Finally, in Sec. II C we derive the general formula (1.2) discussed in the Introduction for the angle- 
averaged, matched-filtering SNR for a gravitational-wave source.

\section{A. Searches for gravitational-wave bursts: Band-pass filtering and matched filtering}

Suppose that some arbitrary gravitational-wave burst $h(t)$ is present in the data stream $s(t)$, so that

$$
s(t)=h(t)+n(t),
$$

where $n(t)$ is noise. If one integrates a filter $K(t)$ against $s(t)$ to produce a number, $Y=\int K(t) s(t) d t$, then the standard definition of the SNR is [61]

$$
\begin{aligned}
\frac{S}{N} & =\frac{\text { expected value of } Y \text { when signal present }}{\text { rms value of } Y \text { when no signal present }} \\
& =\frac{\langle Y\rangle}{\sqrt{\left\langle Y^{2}\right\rangle_{s=0}}} \\
& =\frac{4 \int_{0}^{\infty} d f \Re\left[\widetilde{h}(f)^{*} \widetilde{K}(f)\right]}{\sqrt{4 \int_{0}^{\infty} d f|\widetilde{K}(f)|^{2} S_{h}(f)}} ;
\end{aligned}
$$

see, e.g., Refs. [58,59]. Here tildes denote Fourier transforms according to the convention

$$
\widetilde{h}(f)=\int_{-\infty}^{\infty} e^{2 \pi i f t} h(t) d t,
$$

and $S_{h}(f)$ is the power spectral density of strain noise in the detector [61].

Now consider searching for a signal $h(t)$ when the only information one has about it is its approximate bandwidth in the frequency domain. Perhaps the simplest search algorithm one could use to search for $h(t)$ is to choose for $K(t)$ the following band-pass filter:

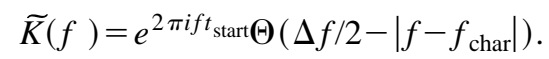

Here $\Theta$ is the step function and $t_{\text {start }}$ is the starting time of the filter. This filter chops out all the data in the frequency domain except that in a bandwidth $\Delta f$ about a characteristic central frequency $f_{\text {char }}$ [71]. Suppose that the frequency interval has been chosen wisely, so that the signal $h(t)$ has negligible power outside the interval. Then $\widetilde{h}(f)$ can be taken to vanish outside the chosen bandwidth, and Eqs. (2.2) and (2.4) yield

$$
\left(\frac{S}{N}\right)_{\text {band-pass }}=\frac{h\left(t_{\text {start }}\right)}{\sqrt{\int_{\Delta f} d f S_{h}(f)}} \approx \sqrt{\frac{f_{\text {char }}}{\Delta f}} \frac{h\left(t_{\text {start }}\right)}{h_{\text {rms }}\left(f_{\text {char }}\right)},
$$

where $h_{\mathrm{rms}}(f) \equiv \sqrt{f S_{h}(f)}$ is the rms fluctuation in the noise at frequency $f$ in a bandwidth equal to $f$. The starting time of the filter, $t_{\text {start }}$, is then varied to give the maximum filter output $Y$, which is achieved at some value $t_{\text {best }}$ of $t_{\text {start }}$. At this maximum overlap time, the SNR is given by Eq. (2.5) with $t_{\text {start }}$ replaced by $t_{\text {best }}$. In particular, for broadband signals for which $\Delta f \sim f_{\text {char }}$, Eq. (2.5) simplifies to the standard result [40]

$$
\left(\frac{S}{N}\right)_{\text {band-pass }} \approx \frac{h\left(t_{\text {best }}\right)}{h_{\text {rms }}\left(f_{\text {char }}\right)} .
$$

By contrast, if the shape of the signal is known, one can use the well-known optimal or matched filter $\widetilde{K}(f)$ $=\widetilde{h}(f) / S_{h}(f)[58,59,61]$ :

$$
\rho^{2}=\left(\frac{S}{N}\right)_{\text {matched }}^{2}=4 \int_{0}^{\infty} \frac{|\widetilde{h}(f)|^{2}}{S_{h}(f)} d f
$$

A crucial element of both matched filtering searches and most especially band-pass filtering searches with groundbased interferometers is the use of coincidencing between different interferometers to circumvent the effects of nonGaussian noise bursts [40]. Coincidencing between the 4 interferometers in the LIGO-VIRGO network (the Hanford 2 $\mathrm{km}$, Hanford $4 \mathrm{~km}$, Livingston $4 \mathrm{~km}$ and Pisa $3 \mathrm{~km}$ interferometers) should be sufficient to achieve this. To be conservative, our assumed detection thresholds for the SNR values are based on combining just the two LIGO $4 \mathrm{~km}$ interferometers, albeit with assumed Gaussian statistics.

In order for a signal to be detected with matched filtering, the waves' measured SNR must be larger than the detection threshold $\rho_{\text {threshold }}$ given by

$$
\operatorname{erfc}\left(\rho_{\text {threshold }} / \sqrt{2}\right)=\frac{\epsilon}{\mathcal{N}_{\text {start-times }} \mathcal{N}_{\text {shapes }}} ;
$$

see, for example, Ref. [44]. Here $\epsilon$ is the false alarm probability, which we will assume below to be $10^{-3}$, corresponding to a false alarm rate of once per $1000 \mathrm{yr}$ if the length of the data set is $1 \mathrm{yr}$. The quantity $\mathcal{N}_{\text {start-times }}$ is the number of independent starting times of the gravitational wave signal that are searched for in the data set, determined by the total duration of the data set (of order one year) and the sampling time. The quantity $\mathcal{N}_{\text {shapes }}=\mathcal{N}_{\text {shapes }}\left(\rho_{\text {threshold }}\right)$ is the number of statistically independent waveforms with $\mathrm{SNR} \leqslant \rho_{\text {threshold }}$ in the set of signals to be searched for [72]; Eq. (2.8) must be solved self-consistently to determine $\rho_{\text {threshold }}$. To a good approximation, Eq. (2.8) reduces to

$$
\rho_{\text {threshold }} \approx \sqrt{2 \ln \left(\mathcal{N}_{\text {start-times }} / \epsilon\right)+2 \ln \left(\mathcal{N}_{\text {shapes }}\right)} .
$$

Typical values of these parameters are $\epsilon=10^{-3}$, a sampling time of $0.01 \mathrm{~s}$ and a data set of $1 \mathrm{yr}$ duration; for these values $\mathcal{N}_{\text {start-times }} / \epsilon \sim 3 \times 10^{12}$ and thus the value of the threshold (2.9) depends only weakly on $\mathcal{N}_{\text {shapes }}$ since $\mathcal{N}_{\text {shapes }} \ll 10^{12}$.

There is a standard lore that the matched-filtering SNR (2.7) is larger than the band-pass filtering SNR (2.6) by approximately the square root of the number of cycles in the waveform $[1,40]$. This relation is strictly speaking only applicable to waveforms that are almost monochromatic, i.e., of the form $h(t)=h_{\mathrm{amp}}(t) \cos [\Phi(t)]$, where the amplitude $h_{\mathrm{amp}}(t)$ and instantaneous frequency [given by $2 \pi f(t)$ $=d \Phi / d t]$ are slowly evolving. The standard lore relation can be obtained by inserting the stationary phase approximation to the Fourier transform of $h(t)$ into Eq. (2.7), which yields

$$
\rho^{2}=\int d(\ln f) n_{\mathrm{cyc}}(f) \frac{h_{\mathrm{amp}}[t(f)]^{2}}{h_{\mathrm{rms}}(f)^{2}},
$$


where $n_{\text {cyc }}(f) \equiv f^{2} / \dot{f}$ is the number of cycles spent within a bandwidth $\Delta f \sim f$ centered on $f$, and $t(f)$ is the time at which the gravitational-wave frequency is $f$. Comparing Eqs. (2.6) and (2.10), we see that $n_{\text {cyc }}(f)$ is the gain factor in SNR squared for matched filtering over band-pass filtering, per logarithmic interval in frequency [1].

This analysis does not apply to signals which are not quasi-monochromatic. We can, however, derive an approximate formula for the SNR (2.6) for general signals. Approximating $S_{h}(f)$ to be constant in Eq. (2.7) gives [40]

$$
\begin{aligned}
\left(\frac{S}{N}\right)_{\text {matched }}^{2} & \approx \frac{2}{S_{h}\left(f_{\text {char }}\right)} \int_{-\infty}^{\infty} d t[h(t)]^{2} \\
& \approx 2 f_{\text {char }} T \frac{\bar{h}^{2}}{h_{\text {rms }}\left(f_{\text {char }}\right)^{2}}
\end{aligned}
$$

where $\bar{h}$ is an rms average of $h(t)$ and $T$ is the effective duration of the signal. Comparing Eqs. (2.11) and (2.5) we find that

$$
\frac{(S / N)_{\text {band-pass }}}{(S / N)_{\text {matched }}} \approx \frac{h\left(t_{\text {best }}\right)}{\bar{h}} \frac{1}{\sqrt{\mathcal{N}_{\text {bins }}}},
$$

where

$$
\mathcal{N}_{\text {bins }}=2 T \Delta f \text {. }
$$

The quantity $\mathcal{N}_{\text {bins }}$ can be interpreted as the "number of $a$ priori frequency bins," since when one searches for a signal of duration $\leqslant T$ and bandwidth $\leqslant \Delta f$, the relevant data is described by $\mathcal{N}_{\text {bins }}$ real Fourier coefficients or, equivalently, frequency bins.

This notion of number of a priori frequency bins is closely related to the notion of number of cycles in the waveform: the number of waveform cycles, $\mathcal{N}_{\text {cyc }} \approx T f_{\text {char }}$, is roughly equal to $\mathcal{N}_{\text {bins }}$ for a broadband burst with $f_{\text {char }}$ $\sim \Delta f$. An important distinction, however, is that $\mathcal{N}_{\text {cyc }}$ is intrinsic to the signal, whereas $\mathcal{N}_{\text {bins }}$ depends upon the characteristics of our band-pass filter. The number of frequency bins thus characterizes in part our a priori assumptions about the signal.

The first factor on the right hand side of Eq. (2.12) is the ratio between the peak strain amplitude $h\left(t_{\text {best }}\right)$ in the time domain and an rms value $\bar{h}$ of this strain amplitude. By defining the effective duration $T$ of the signal to be given by

$$
\int d t[h(t)]^{2}=T h\left(t_{\text {best }}\right)^{2},
$$

this factor reduces to unity. With this interpretation of $T$ in Eq. (2.13), Eq. (2.12) reduces to

$$
\frac{(S / N)_{\text {band-pass }}}{(S / N)_{\text {matched }}} \approx \frac{1}{\sqrt{\mathcal{N}_{\text {bins }}}},
$$

as discussed in the Introduction. We use this result in Sec. VI B.

\section{B. Searches for gravitational-wave bursts: Noise monitoring}

In this section we describe a "noise-monitoring" method to search for gravitational wave bursts of unknown form; more details can be found in Ref. [73]. A variant of the method was first suggested by Schutz [40] (there called an autocorrelation method); here we modify slightly Schutz's suggestion and also calculate detection thresholds. In essence, the method consists of monitoring the total rms noise in the detector output in the frequency band in which the signal is expected, rms averaged over time scales of the expected signal duration, and waiting for statistically significant changes in one's estimate of the noise power.

Suppose that the maximum expected signal duration is $T$, and that the interferometer output is $s(t)$. Focus attention on the data stream $s(\tau)$ in the time interval $t-T / 2 \leqslant \tau \leqslant t$ $+T / 2$. Since the data stream is discrete, this data can be represented by the numbers

$$
s_{j}=s(t-T / 2+j \Delta t)
$$

for $0 \leqslant j \leqslant N_{\text {total }}=T / \Delta t$, where $\Delta t$ is the sampling time. From Eq. (2.1) we have

$$
s_{j}=h_{j}+n_{j},
$$

where $h_{j}$ is the gravitational-wave signal and $n_{j}$ is the noise. Now because the interferometer noise is colored, the noise matrix

$$
\Sigma_{i j} \equiv\left\langle n_{i} n_{j}\right\rangle
$$

will not be diagonal. Here, angular brackets denote ensemble averaging over realizations of the noise. If one performs a fast Fourier transform (FFT) just of this finite stretch of data, the noise matrix on the new basis will not be diagonal either because of aliasing effects. However, it is possible to change to a basis which diagonalizes the matrix (2.18). We will denote this new basis by capital Roman letters $I, J, K$. The data points $s_{I}$ on this new basis can be chosen to correspond approximately to frequencies $f_{I}=I / T, \quad I=1,-1,2,-2, \ldots$. [73]. Equation (2.18) can now be replaced by

$$
\left\langle n_{I} n_{J}\right\rangle=\delta_{I J} \sigma_{I}^{2} .
$$

The data $s_{I}$ extend up to some high frequency (of order several $\mathrm{kHz}$ ) determined by the sampling time. We next discard all data above some upper cutoff frequency; thus, we have effectively band-pass filtered the data, since the restriction to a segment of length $T$ in the time domain removes frequency components at $f \lesssim 1 / T$. The total number of data points remaining will be approximately $\mathcal{N}_{\text {bins }}=2 T \Delta f$, where $\Delta f$ is the bandwidth of our effective band-pass filter.

In terms of this new basis, matched filtering consists of calculating, for each trial waveform shape $h_{J}$, the quantity

$$
\frac{\Sigma_{J} S_{J} h_{J} / \sigma_{J}^{2}}{\sqrt{\Sigma_{J} h_{J}^{2} / \sigma_{J}^{2}}} .
$$

(We are assuming here that all the trial waveform shapes have duration less than $T$ and most of their power within the bandwidth $\Delta f$.) We introduce the notation $\rho_{I}=h_{I} / \sigma_{I}$; then, the matched filtering SNR (1.1) becomes 


$$
\rho^{2}=\sum_{I} \rho_{I}^{2}=\sum_{I} \frac{h_{I}^{2}}{\sigma_{I}^{2}}
$$

Thus, the quantity $\rho_{I}^{2}$ is the matched filtering SNR-squared per data bin. Throughout this subsection, we use $\rho$ as a convenient parameterization of the signal strength, which is meaningful even in situations where templates are not available and where matched filtering cannot be carried out.

In this language, band-pass filtering (of a pre-whitened data stream) approximately corresponds to calculating the statistic

$$
\hat{\rho}_{\mathrm{BP}} \equiv \max _{J} \frac{s_{J}}{\sigma_{J}} .
$$

This will have an expected value of $\sim \rho / \sqrt{\mathcal{N}_{\text {bins }}}$ [cf. Eq. (2.15)] if the signal is spread out over the bandwidth $\Delta f$ rather than peaked at some frequency.

In the noise-monitoring technique, the detection statistic is

$$
Q(t)=-\mathcal{N}_{\text {bins }}+\sum_{J=-\mathcal{N}_{\text {bins }} / 2}^{J=\mathcal{N}_{\text {bins }} / 2} \frac{s_{J}^{2}}{\sigma_{J}^{2}}
$$

Up to an additive constant, $Q(t)$ is an estimate of the noise power in the given bandwidth over the given time interval. That constant, $-\mathcal{N}_{\text {bins }}$, is chosen so that when no signal is present, $\langle Q(t)\rangle=0$ and so $Q(t)$ fluctuates between positive and negative values. On the other hand, when a signal is present, $Q(t)$ will with high probability be large and positive. One monitors $Q(t)$ as a function of time, setting a threshold that it has a very low probability of exceeding in the absence of a signal. This search method constitutes a type of nonlinear filtering.

Noise-monitoring is closely related to two commonly used techniques in radio astronomy. In the first such technique, observers sum the power from frequency bins which are expected to contain harmonics of the signal they are trying to detect. This procedure is not as effective as coherently combining the signal from all the frequency bins, but is computationally much easier. The second technique [74] is applicable when one is looking for periodic signals in a data train that is too long to Fourier transform. One splits the data into shorter segments, takes the FFT of each segment, and adds the FFTs incoherently (i.e., adds the individual power spectra). This is not the optimal search method, but is often useful given finite computational resources. Although noisemonitoring and the radio astronomy techniques have different motivations (in radio astronomy, one adds frequency bins incoherently to save computational cost; in noise-monitoring, one performs such an addition because the phase relationships are unknown), they are operationally quite similar.

We now turn to a derivation of the efficiency and performance of the method. From Eq. (2.23), when a signal is present,

$$
\begin{gathered}
\langle Q(t)\rangle=\rho^{2}, \\
\left\langle[Q(t)-\langle Q(t)\rangle]^{2}\right\rangle=4 \rho^{2}+2 \mathcal{N}_{\text {bins }} .
\end{gathered}
$$

With no signal present, Eqs. (2.24) continue to hold with $\rho$ $=0$. These equations show that a signal should be detectable in the regime

$$
\mathcal{N}_{\text {bins }}^{1 / 4} \ll \rho \lesssim \mathcal{N}_{\text {bins }}^{1 / 2},
$$

as well as at larger $\rho$ : in the regime (2.25) the expected value (2.24) of $Q$ is large compared to its rms value in the absence of a signal. By contrast, a signal is detectable using bandpass filtering only in the regime $\rho \geq \mathcal{N}_{\text {bins }}^{1 / 2}$ [Eq. (2.22) above and associated discussion].

The approximate SNR threshold predicted by Eqs. (2.24) is correct in order of magnitude, but to obtain an accurate SNR threshold one needs to calculate the full probability distribution for the statistic $Q$. This probability distribution is given by, from Eqs. (2.17), (2.19) and (2.23),

$$
P\left[Q(t) \geqslant Q_{0}\right]=\frac{\Gamma\left(\mathcal{N}_{\text {bins }} / 2,\left(Q_{0}+\mathcal{N}_{\text {bins }}\right) / 2\right)}{\Gamma\left(\mathcal{N}_{\text {bins }} / 2\right)}
$$

where $\Gamma(\cdots, \cdots)$ is the incomplete gamma function and $\Gamma(\cdots)$ is the usual gamma function. Suppose that we examine $\mathcal{N}_{\text {start-times }}$ starting times $t$. We wish to find the number $Q_{0}$ such that the probability (2.26) of $Q(t)$ exceeding $Q_{0}$ for any $t$, in the absence of a signal, is some small number $\epsilon$ (below we will take $\epsilon=10^{-3}$ ). This threshold $Q_{0}$ is obtained by solving

$$
\frac{\Gamma\left(\mathcal{N}_{\text {bins }} / 2,\left(Q_{0}+\mathcal{N}_{\text {bins }}\right) / 2\right)}{\Gamma\left(\mathcal{N}_{\text {bins }} / 2\right)}=\frac{\epsilon}{\mathcal{N}_{\text {start-times }}} .
$$

From Eqs. (2.24), this threshold will be exceeded by a signal whenever the signal strength (2.21) satisfies

$$
\rho \geqslant \rho_{*}=\sqrt{Q_{0}} .
$$

Equations (2.27) and (2.28) determine the threshold $\rho_{*}$ as a function of the parameters $\epsilon, \mathcal{N}_{\text {start-times }}$, and $\mathcal{N}_{\text {bins }}$; we use these formulas in Sec. VI B. For $\mathcal{N}_{\text {bins }} \gg 1, \rho_{*}$ is approximately given by solving the equation

$$
\rho_{*}^{2}=2 \ln \left(\mathcal{N}_{\text {start-times }} / \epsilon\right)+\mathcal{N}_{\text {bins }} \ln \left(1+\rho_{*}^{2} / \mathcal{N}_{\text {bins }}\right) .
$$

The above derivation is based on frequentist statistics. In Ref. [73] a Bayesian analysis is outlined of the detection of gravitational wave signals of unknown form which automatically identifies the statistic $Q(t)$ as optimal, and which also approximately reproduces the detection threshold $\rho_{*}$.

In practice, this search method would be combined with coincidencing between interferometers to achieve high detection reliability and to reduce the effects of non-Gaussian noise, as is the case with band-pass and matched filtering discussed above. Matched filtering could be more efficient than the noise-monitoring method at combating nonGaussian noise via coincidencing: when coincidencing with templates, one can demand that the SNR in each interferometer be above the appropriate threshold, and that the signalparameter values deduced in each interferometer be consistent with each other. For the noise-monitoring searches, one can only demand that the SNR in each interferometer be above the appropriate threshold. Hence, matched filtering has 
more discriminating power against situations in which all the interferometers have moderately large non-Gaussian noise spikes somewhere in the relevant time window. NonGaussian noise may therefore make the less-discriminating noise-monitoring search perform somewhat worse in practice, relative to matched filtering searches, than is indicated by the threshold (2.29).

\section{Signal-to-noise ratio for matched filtering in terms of waves' energy spectrum}

In this section we derive the relation (2.30) between the expected value of the matched-filtering SNR (2.7) and the energy spectrum of emitted gravitational waves. In general, the SNR (2.7) for a burst of waves depends on the details of the gravitational waveform, on the orientation of the source with respect to the interferometer, and on the direction to the source. By contrast, the quantity $\left\langle\rho^{2}\right\rangle$, the average of the squared SNR over all orientations of and directions to the source, depends only on the total energy per unit frequency $d E / d f$ carried off from the source by the waves. Consider a gravitational-wave source located at a cosmological redshift $z$ and corresponding luminosity distance $D(z)$. Let the locally measured frequency of the waves near the source be $f_{e}$, related to the frequency $f$ measured at the interferometer by $f=f_{e} /(1+z)$. Let the locally measured energy spectrum of the waves be $d E_{e} / d f_{e}\left(f_{e}\right)$. Then the orientation-averaged SNR squared is given by

$$
\left\langle\rho^{2}\right\rangle=\frac{2(1+z)^{2}}{5 \pi^{2} D(z)^{2}} \int_{0}^{\infty} d f \frac{1}{f^{2} S_{h}(f)} \frac{d E_{e}}{d f_{e}}[(1+z) f] .
$$

Note that the relation (2.30) refers to an angle-averaged SNR obtained from an rms average of signal amplitudes over different possible orientations of the source and interferometer. This averaging convention differs from that adopted in Refs. [1,10], where the angle-averaged SNR is taken to be a cube root of an average of cubed signal amplitudes. That "cube root of a mean cube" method is appropriate for calculating the expected event detection rate [1]. As a result, the SNR formulas used in Refs. [1,10] are a factor of $\sqrt{3 / 2}$ larger than those used in this paper, the factor of $\sqrt{3 / 2}$ being an approximation to the effect of the different angleaveraging methods.

Turn now to the derivation of Eq. (2.30). First, consider a source close enough that cosmological effects can be neglected. Let the source be at a distance $r$ from the detector and at a location $(\theta, \varphi)$ on the sky. Let $(\iota, \beta)$ denote the direction towards the detector (spherical polar angles) with respect to a set of Cartesian axes centered at and determined by the source. Let the two independent polarizations of the strain amplitude at the interferometer be $h_{+}(t, r, \iota, \beta)$ and $h_{\times}(t, r, \iota, \beta)$, and let the polarization angle be $\psi$. Then the response of the interferometer will be $h(t)+n(t)$, where $n(t)$ is the noise, and

$$
h(t)=F_{+}(\theta, \varphi, \psi) h_{+}(t, r, \iota, \beta)+F_{\times}(\theta, \varphi, \psi) h_{\times}(t, r, \iota, \beta) .
$$

Here $F_{+}$and $F_{\times}$are the interferometer beam pattern functions, given in, e.g., Ref. [1]. The dependence of the Fourier transformed waveform $\widetilde{h}_{+}$on $r$ is of the form

$$
\widetilde{h}_{+}(f, r, \iota, \beta)=H_{+}(f, \iota, \beta) / r
$$

for some function $H_{+}$; we define $H_{\times}(f, \iota, \beta)$ similarly. Combining Eqs. (2.7), (2.31) and (2.32) gives

$$
\rho^{2}(r, \theta, \varphi, \psi, \iota, \beta)=\frac{4}{r^{2}} \int_{0}^{\infty} \frac{\left|F_{+} H_{+}+F_{\times} H_{\times}\right|^{2}}{S_{h}(f)} d f .
$$

We now average over the angles $\theta, \varphi, \psi, \iota$ and $\beta$. The average over polarizations and over the sky location gives $\left\langle F_{+}^{2}\right\rangle=\left\langle F_{\times}^{2}\right\rangle=1 / 5,\left\langle F_{+} F_{\times}\right\rangle=0[1]$, where the meaning of the angular brackets is given by, for example,

$$
\left\langle F_{+}^{2}\right\rangle \equiv \frac{1}{4 \pi} \int d \Omega_{\theta, \varphi} \int_{0}^{\pi} \frac{d \psi}{\pi} F_{+}(\theta, \varphi, \psi)^{2} .
$$

From Eq. (2.33) this gives

$$
\left\langle\rho^{2}\right\rangle=\frac{4}{5 r^{2}} \int_{0}^{\infty} \frac{H(f)^{2}}{S_{h}(f)} d f
$$

where

$$
H(f)^{2} \equiv \frac{1}{4 \pi} \int d \Omega_{\iota, \beta}\left[\left|H_{+}(\iota, \beta)\right|^{2}+\left|H_{\times}(\iota, \beta)\right|^{2}\right] .
$$

We now express the energy spectrum $d E / d f$ of the waves in terms of the quantity $H(f)^{2}$. The local energy flux is

$$
\frac{d E}{d A d t}=\frac{1}{16 \pi} \overline{\left[\left(\frac{\partial h_{+}}{\partial t}\right)^{2}+\left(\frac{\partial h_{\times}}{\partial t}\right)^{2}\right]},
$$

where the overbar means an average over several cycles of the wave. Switching to the frequency domain using Parseval's theorem, inserting a factor of 2 to account for the folding of negative frequencies into positive, and using $\left|\widetilde{h}_{+, \times}(f)\right|^{2} d A=\left|\widetilde{H}_{+, \times}(f)\right|^{2} d \Omega$ gives

$$
\frac{d E}{d \Omega d f}=\frac{\pi f^{2}}{2}\left[\left|\widetilde{H}_{+}(\iota, \beta)\right|^{2}+\left|\widetilde{H}_{\times}(\iota, \beta)\right|^{2}\right] .
$$

Combining Eqs. (2.35), (2.36) and (2.38) now yields

$$
\left\langle\rho^{2}\right\rangle=\frac{2}{5 \pi^{2} r^{2}} \int_{0}^{\infty} d f \int d \Omega \frac{1}{f^{2} S_{h}(f)} \frac{d E}{d \Omega d f}(f) .
$$

This is Eq. (2.30) with $z=0$ and $D(z)=r$, the limiting form that applies when cosmological effects are neglected.

Consider now sources at cosmological distances. First, observe that Eq. (2.39) is valid for arbitrary bursts of gravitational waves provided that we interpret the quantity

$$
\frac{1}{r^{2}} \frac{d E}{d \Omega d f}
$$


as the locally measured energy flux $d E / d A d f$. Next, note that the number of gravitons per unit solid angle per unit frequency is conserved for propagation in a FriedmannRobertson-Walker background in the geometric optics limit:

$$
\frac{d E}{d \Omega d f}(f)=\frac{d E_{e}}{d \Omega d f_{e}}[(1+z) f] .
$$

Here $f_{e}$ is the frequency at the source and $f=f_{e} /(1+z)$ is the frequency at the detector. Finally, the conversion factor at the detector from energy per unit solid angle to energy per unit area is $(1+z)^{2} / D(z)^{2}$, where $D(z)$ is the luminosity distance [75]. Hence

$$
\frac{d E}{d A d f}(f)=\frac{(1+z)^{2}}{D(z)^{2}} \frac{d E_{e}}{d \Omega d f_{e}}[(1+z) f] .
$$

Combining this with Eq. (2.39) yields Eq. (2.30).

\section{GRAVITATIONAL-WAVE SIGNAL FROM COALESCING BLACK HOLES}

In this section we describe our assumptions concerning the gravitational-wave signal from BBH mergers and the evidence that underlies those assumptions.

\section{A. Three phases of the gravitational-wave signal}

As discussed in the Introduction, the coalescence and its associated gravitational-wave signal can be divided into three successive epochs in the time domain: inspiral, merger, and ringdown. The inspiral consists of the coalescence epoch in which the black holes are separated bodies that gradually lose energy and angular momentum, slowly spiraling towards one another. The merger is the epoch in which the dynamics is highly nonlinear and must be treated by numerical relativity. With this in mind, it is useful to define the end of inspiral as the time and frequency at which numerically generated templates become needed [76]. Up to this time, post-Newtonian templates, possibly supplemented with IBBH templates, will be used (cf. Sec. I B).

After merger, the system will gradually settle down to a Kerr black hole; the last gravitational waves we expect to see are those produced by the quasi-normal modes of this merged black hole. It is clear that there will be a smooth transition in the gravitational waveform from the merger portion to the ringdown portion, as the effects of nonlinearities become less and less important with time. As this happens, the signal should become increasingly well approximated by a linear combination of exponentially decaying sine waves. This is the behavior that has been seen in numerical simulations of, for example, head-on collisions [77,78]. At late times, the $l=m=2$ mode will probably dominate over other quasi-normal modes, for two reasons which are of comparable importance: (i) The $l=m=2$ mode is the most slowly damped of all the QNR modes [14], and (ii) during coalescence, the binary will have a rotating shape roughly corresponding to spheroidal harmonic indices $l=m=2$, so this mode will be preferentially excited [79]. We define the ringdown as beginning when the waveform becomes dominated by the $l=m=2$ QNR mode; the merger thus contains those portions of the waveform where other modes and/or non- linear mode-mode couplings are important. Clearly there is some arbitrariness in the exact time at which the ringdown starts, related to the accuracy we require of the fit of the waveform to the ringdown signal.

By definition, the three phases of the signal are disjoint in the time domain. It does not follow that they should be disjoint in frequency: their energy spectra might overlap. However, it is at least approximately true that the inspiral and merger are disjoint in both time and frequency. The adiabatic approximation is only just beginning to break down at the end of inspiral; thus, there is a well-defined frequency as a function of time $f(t)$ over almost the entire inspiral. Because the inspiral chirps upward monotonically in frequency, almost all energy emitted before the merger lies at frequencies less than $f_{\text {merge }}$, the gravitational-wave frequency at the end of inspiral. We discuss below estimates of $f_{\text {merge }}$. We shall assume that the merger waves' spectrum is confined to the frequency regime $f>f_{\text {merge }}$. One particular component of the gravitational-wave signal, the Christodoulou memory [80], will violate this assumption. This component has most of its power below $f_{\text {merge }}$ in the frequency domain, but accumulates gradually during the inspiral, merger and ringdown in the time domain. It will probably not be detectable with ground-based interferometers, but very probably will be detectable with LISA [81]. We will neglect the memory component of the waves in our analysis, since it will not be as easy to detect as the components we do discuss.

\section{B. Energy spectrum of the emitted gravitational radiation from the merger phase}

The total amount of energy radiated in BBH mergers, and its distribution in frequency, is highly uncertain because detailed numerical calculations of these mergers have not yet been made. In this subsection, we discuss what little evidence there is about the energy radiated, and describe our crude model of the spectrum.

The total amount of energy radiated during a BBH coalescence will be some fraction $\epsilon$ of the total mass $M=m_{1}$ $+m_{2}$ of the system: $E_{\text {radiated }}=\epsilon M$. The fraction $\epsilon$ will depend only on the mass ratio $m_{1} / m_{2}$, on the initial spins $\mathbf{S}_{1}$ and $\mathbf{S}_{2}$ of the two black holes, and on the initial direction $\hat{\mathbf{L}}$ of the orbital angular momentum [82]:

$$
\epsilon=\epsilon\left(\frac{m_{1}}{m_{2}}, \frac{\mathbf{S}_{1}}{M^{2}}, \frac{\mathbf{S}_{2}}{M^{2}}, \hat{\mathbf{L}}\right) .
$$

We can very roughly divide up this fraction as

$$
\epsilon=\epsilon_{\text {inspiral }}+\epsilon_{\text {merger }}+\epsilon_{\text {ringdown }},
$$

according to the amounts of energy radiated in the three different epochs of the waveform. We emphasize that there is some arbitrariness in this division, related to the choice of frequency at the end of inspiral and the time at the beginning of ringdown.

We now discuss estimates of the frequency $f_{\text {merge }}$. From a data-analysis oriented viewpoint, $f_{\text {merge }}$ should represent the frequency at which post-Newtonian templates cease to be useful and numerical templates will be needed. On the other hand, $f_{\text {merge }}$ could be chosen at the supposed point of transition from a radiation-reaction driven inspiral to a freely fall- 
ing plunge $[8,11,12]$. These two viewpoints turn out to give roughly the same value for $f_{\text {merge }}$.

To estimate the frequency where numerical templates are likely to be needed, we examined numerical initial data sets of black holes binaries generated by Cook [9]. Comparing the predictions of second post-Newtonian order calculations to his initial data sets, Cook finds that the discrepancy in the binding energy between the two methods is $\sim 5 \%$ at $f$ $\approx 0.02 / M$ (where $M$ is the total system mass) and is $\sim 15 \%$ at $f \approx 0.05 / M$. Thus, numerical relativity's predictions begin to significantly deviate from post-Newtonian theory near $f$ $=0.02 / M$.

The "innermost stable circular orbit" (ISCO) for black hole binaries only exists, strictly speaking, in the test particle limit $m_{1} \ll m_{2}$, and it is not clear that it is well defined, even approximately, in the equal mass case. Nevertheless, various methods have been proposed to locate the supposed transition point from inspiral to plunge. Cook estimates the gravitational wave frequency at the ISCO to be $f_{\text {ISCO }} \sim 0.055 / M$ for equal mass black holes [9], by using his initial data sets together with the calculation of an "effective potential." In post-Newtonian theory, the ISCO can be defined by artificially turning off the radiation reaction terms in the equations of motion. Using this method, Kidder, Will and Wiseman estimate $f_{\text {ISCO }} \sim 0.02 / M$ [8]. This value varies by less than $\sim 20 \%$ as the mass ratio is varied. Finally, earlier analyses by Blackburn and Detweiler used a variational principle together with the assumption of periodic solutions to Einstein's equations to obtain the approximate lower bound $f_{\text {ISCO }}$ $\gtrsim 0.06 / M$ [83]. All of these estimates are for equal mass, non-spinning black holes; the value of the frequency $f_{\text {ISCO }}$ can presumably also vary by factors of $\geqslant 2$ if the black holes are spinning and/or have different masses.

Given this uncertainty, we adopt the conservative value

$$
f_{\text {merge }}=\frac{0.02}{M}=205 \mathrm{~Hz}\left(\frac{20 M_{\odot}}{M}\right) .
$$

This (low) value of $f_{\text {merge }}$ is conservative in the sense that we can be reasonably sure numerically generated templates will not be needed before $f=f_{\text {merge }}$. On the other hand, it may overestimate the merger SNR by increasing the number of cycles in what we define as our merger waveform at the expense of the inspiral.

We next discuss our choice of upper frequency shutoff for the merger energy spectrum. As discussed above, we define the end of merger to occur at a time $t_{\mathrm{qnr}}$ after which the waveform can be accurately fit by the $l=m=2$ QNR signal. The merger and ringdown will therefore be disjoint in the time domain, but not necessarily in the frequency domain. It seems likely, however, that an approximate upper bound for the frequencies carrying appreciable power during the merger is the quasinormal ringing frequency itself. This conjecture is supported by calculations in the test particle limit (cf. Fig. 2 of Ref. [68]) and calculations of the head-on collision of two black holes [77,78]. It is not clear how relevant these calculations are to the merger of comparable mass black holes, but there is no other guidance available at this time.

Therefore, we use the frequency $f_{\text {qnr }}$ of the $l=m=2$ quasi-normal mode as our upper merger frequency. This fre- quency depends on the dimensionless spin parameter $a$ of the final Kerr black hole; for concreteness, we use the value of $a=.98$, for which $f_{\mathrm{qnr}} \simeq 0.9 /(2 \pi M)[56,15]$ :

$$
f_{\mathrm{qnr}}=\frac{0.13}{M}=1320 \mathrm{~Hz}\left(\frac{20 M_{\odot}}{M}\right) .
$$

Our reasons for assuming a high value of $a$ are discussed in Sec. III D below.

Finally, consider the total amount of energy

$$
E_{\text {rad }}=\left(\epsilon_{\text {merger }}+\epsilon_{\text {ringdown }}\right) M
$$

radiated during the final merger and ringdown. We consider two methods of estimating this radiation efficiency, which yield consistent results. The first method, due to Smarr $[67,77]$, is an extrapolation from perturbation theory: the energy radiated in the test particle limit is of the form

$$
E_{\mathrm{rad}}=k \mu^{2} / M,
$$

where $k$ is a dimensionless constant, $\mu$ is the mass of the particle, and $M$ the total mass of the system. Replacing $\mu$ by the reduced mass of the system, one finds that the formula (3.6) reliably predicts (to within $\sim 20 \%$ ) the energy radiated in the head-on collision of two black holes $[68,77,78,84]$. Consider applying a similar extrapolation to an inspiralpreceded merger. Detweiler [68] examined the amount of energy radiated per orbit by a test particle on the final, marginally bound orbit of a Kerr black hole. He found that the energy radiated is of the form (3.6), with $0.65 \leqslant k \leqslant 2.8$ as the spin of the black hole varies from 0 to .95 . Assuming that there will be $\gtrsim 1$ effective orbit during the final plunge, Detweiler estimates [68]

$$
0.03 M F(\mu / M) \lesssim E_{\mathrm{rad}} \lesssim 0.2 M F(\mu / M),
$$

where $F(\mu / M)$ is given in Eq. (1.5).

A second method, based on angular momentum conservation, also suggests a lower bound on $E_{\text {rad }}$ of about $0.1 M$ for equal-mass $\mathrm{BBHs}$ in the most favorable cases. Roughly speaking, the system's angular momentum divides up as

$$
\mathbf{S}_{1}+\mathbf{S}_{2}+\mathbf{L}_{\mathrm{orb}}=\mathbf{J}_{\mathrm{rad}}+\mathbf{S}_{\mathrm{final}},
$$

where $\mathbf{S}_{1}$ and $\mathbf{S}_{2}$ are the black hole spins just before the final plunge, $\mathbf{L}_{\text {orb }}$ is the orbital angular momentum just before the plunge, $\mathbf{J}_{\mathrm{rad}}$ is the angular momentum radiated in the merger and ringdown waves, and $\mathbf{S}_{\text {final }}$ is the spin of the final Kerr black hole. This splitting of the spacetime's total angular momentum is, strictly speaking, well defined only in a postNewtonian type of limit; however, the effects of this ambiguity are presumably not important for the purposes of our crude estimate. Specialize now to the most favorable case where $\mathbf{S}_{1}, \mathbf{S}_{2}$ and $\mathbf{L}_{\text {orb }}$ are all aligned. We assume $\left|\mathbf{L}_{\text {orb }}\right|$ $\approx 0.9 M^{2}$, the value predicted by Cook's initial data sets at $f=.02 / M$ [9]. We also assume that both black holes are rapidly spinning, so that $\left|\mathbf{S}_{1}\right| \approx\left|\mathbf{S}_{2}\right| \approx(M / 2)^{2}$. Equation (3.8) then yields

$$
\left|\mathbf{J}_{\text {rad }}\right| \gtrless 0.4 M^{2}
$$


since $\left|\mathbf{S}_{\text {final }}\right| \leqslant M^{2}$. Next, we use that fact that the energy $E_{\text {rad }}$ and the angular momentum $J_{\text {rad }}$ carried off by gravitons of frequency $f$ and azimuthal multipole order $m$ are related by [85]

$$
E_{\mathrm{rad}}=2 \pi f J_{\mathrm{rad}} / m
$$

If we estimate $f \approx\left(f_{\text {merge }}+f_{\text {qnr }}\right) / 2$, and make the admittedly optimistic assumption that most of the radiation is quadrupolar, we obtain from Eqs. (3.9) and (3.10) the estimate [86]

$$
E_{\text {rad }} \gtrsim 0.1 M \text {. }
$$

This estimate includes both merger and ringdown radiation; we need to subtract the ringdown portion to obtain the energy radiated in the merger. Below we estimate $\sim 0.03 \mathrm{M}$ to be an approximate upper bound for the ringdown energy. Hence most of the energy (3.11) should be radiated as merger waves.

There is an additional, separate argument one can make which indicates that most of the energy (3.11) should be radiated as merger waves and not as ringdown waves. As noted by Eardley and Hirschmann [87], any system with $J$ $>M^{2}$ cannot evolve to $J<M^{2}$ by radiating quadrupolar waves at the ringing frequency $f_{\mathrm{qnr}} \sim 1 /(2 \pi M)$ of a nearextremal Kerr black hole. This is because at this high frequency, too much mass-energy is radiated per unit angular momentum radiated; Eq. (3.10) with $m=2$ and with $f=f_{\text {qnr }}$ yields $\Delta J=\Delta\left(M^{2}\right)$. Hence, since the final black hole must have $J<M^{2}$, a substantial amount of the radiation must be emitted at lower frequencies.

Based on the estimates (3.7) and (3.11), and on the estimated upper bound $\sim 0.03 M$ which we derive below for the ringdown radiated energy, we take $0.1 M$ as our radiated energy for the merger in the equal mass case. For non-equalmass BBHs we assume that the radiated energy is reduced by the factor (1.5), so that

$$
E_{\text {merger }}=\epsilon_{\text {merger }} F(\mu / M) M=0.1 F(\mu / M) M .
$$

This rather high radiation efficiency is probably most plausible in the context of rapidly spinning coalescing black holes. In particular, if the spins and the orbital angular momentum are somewhat misaligned, one would intuitively expect that such systems have more "settling down" to do to get to the final Kerr black hole, and that correspondingly the nonlinear, highly dynamical phase should last longer and/or produce more radiation. Also, the potential barrier that surrounds the final black hole (which normally tends to reflect back into the black hole the dominant waves of frequency $f \sim 1 /$ a few times $M$ ) presumably will effectively not be present during the violent phase of a merger in which the spins and orbital angular momentum are of comparable magnitude and are misaligned.

Coalescences which radiate as much energy as Eq. (3.12) may also radiate a substantial amount of linear momentum; the consequent recoil of the final black hole could correspond to a kick velocity that is a moderate fraction of the speed of light.
Finally, consider the shape of the energy spectrum $d E / d f$ between $f_{\text {merge }}$ and $f_{\text {qnr }}$. For simplicity, and for lack of evidence in favor of anything more specific, we choose a flat spectrum:

$$
\begin{aligned}
\frac{d E}{d f} & =\frac{\epsilon_{m} M F(\mu / M)}{f_{\mathrm{qnr}}-f_{\text {merge }}} \Theta\left(f-f_{\text {merge }}\right) \Theta\left(f_{\text {qnr }}-f\right) \\
& =0.91 M^{2} F(\mu / M) \Theta\left(f-f_{\text {merge }}\right) \Theta\left(f_{\text {qnr }}-f\right),
\end{aligned}
$$

where $\epsilon_{m}=\epsilon_{\text {merger }}=0.1$ and $\Theta$ is the step function.

\section{Energy spectrum of the radiation from the inspiral phase}

The standard quadrupole formula prediction for the inspiral energy spectrum is (see, e.g., Ref. [88])

$$
\frac{d E}{d f}=\frac{1}{3} \pi^{2 / 3} \mu M^{2 / 3} f^{-1 / 3} .
$$

This formula is adequate to estimate the SNR obtained from matched filtering of the inspiral waveform; it will be accurate to within a few tens of a percent up to $f=f_{\text {merge }}$ [63]. Using Eq. (3.14) to estimate the SNR assumes that both the gravitational-wave signal and the templates used to filter the data stream are given by the quadrupole approximation (3.14). The SNR we calculate using Eq. (3.14) will be approximately the same as that found by cross-correlating real signals against sufficiently accurate theoretical templates [which incorporate higher order corrections to Eq. (3.14)]. As outlined in Sec. IB, the required template accuracy should be achievable by post-Newtonian expansions $[48,89]$, perhaps supplemented with alternative techniques for the latter, high frequency part of the signal at $0.01 / M \lesssim f$ $\leq 0.02 / M$ (the IBBH regime). We assume that the inspiral energy spectrum shuts off at $f=f_{\text {merge }}=0.02 / M$, as discussed in Sec. III B above.

\section{Energy spectrum of the radiation from the ringdown phase}

The ringdown of the gravitational-wave signal is that portion which can be fit fairly accurately by an exponentially decaying sinusoid corresponding to the $l=m=2$ quasinormal mode of the final black hole. The shape of the corresponding energy spectrum is well understood: it is a resonance curve (although see Appendix B for discussion of a subtlety in the applicability of the concept of the waves' energy spectrum to calculating ringdown SNRs). The overall amplitude of the energy spectrum, however, is not well understood.

The QNR gravitational waveforms $h_{+}(t, \iota, \beta)$ and $h_{\times}(t, \iota, \beta)$ are given by $[15]$

$$
h_{+}-i h_{\times}=\frac{\mathcal{A} M}{r}{ }_{2} S_{2}^{2}(\iota, \beta, a) e^{-2 i \pi f_{\mathrm{qnr}} t-t / \tau+i \varphi_{0}},
$$

for $t>0$. Here we have chosen $t=0$ to be the start of the ringdown, $M$ is the final black hole mass, $a M^{2}$ is its spin, and $\varphi_{0}$ is a constant phase. The quantities $\iota$ and $\beta$ are spherical polar coordinates centered on the black hole [cf. Sec. 
II C], ${ }_{2} S_{2}^{2}(\iota, \beta, a)$ is a spin weighted spheroidal harmonic whose angle averaged rms value is

$$
\left(\left.\left.\frac{1}{4 \pi} \int d \Omega\right|_{2} S_{2}^{2}(\iota, \beta, a)\right|^{2}\right)^{1 / 2}=\frac{1}{\sqrt{4 \pi}},
$$

and $\mathcal{A}$ is a dimensionless coefficient that describes the magnitude of the perturbation when the ringdown begins. The quantities $f_{\text {qnr }}$ and $\tau$ are the frequency and damping time, respectively, of the $l=m=2$ QNR mode. The quality factor $Q$ of the mode is given by $Q=\pi \tau f_{\mathrm{qnr}}$.

As mentioned in the Introduction, there is a mapping, explored by Leaver [90] and Echeverria [15], between the $(M, a)$, and $\left(f_{\mathrm{qnr}}, \tau\right)$. Using the Teukolsky equation, Leaver produced catalogs of $\tau$ and $f_{\text {qnr }}$ as functions of black hole mass $M$ and dimensionless spin $a$ [90]. From that data, Echeverria [15] produced the following analytic fits, which are good to about $5 \%$ :

$$
\begin{aligned}
f_{\mathrm{qnr}} & \approx\left[1-0.63(1-a)^{3 / 10}\right] \frac{1}{2 \pi M} \\
& =\left[1-0.63(1-a)^{3 / 10}\right]\left(\frac{20 M_{\odot}}{M}\right) 1620 \mathrm{~Hz} \\
Q & \equiv \pi f_{\mathrm{qnr}} \tau \approx 2(1-a)^{-9 / 20} .
\end{aligned}
$$

The energy spectrum for the QNR waveform (3.15) is derived in Appendix A and is given by

$$
\begin{aligned}
\frac{d E}{d f}= & \frac{\mathcal{A}^{2} M^{2} f^{2}}{32 \pi^{3} \tau^{2}}\left\{\frac{1}{\left[\left(f-f_{\mathrm{qnr}}\right)^{2}+(2 \pi \tau)^{-2}\right]^{2}}\right. \\
& \left.+\frac{1}{\left[\left(f+f_{\mathrm{qnr}}\right)^{2}+(2 \pi \tau)^{-2}\right]^{2}}\right\} \\
\approx & \frac{1}{8} \mathcal{A}^{2} Q M^{2} f_{\mathrm{qnr}} \delta\left(f-f_{\mathrm{qnr}}\right)[1+O(1 / Q)]
\end{aligned}
$$

Approximating the energy spectrum by a delta function as in Eq. (3.19) will often (but not always) provide a fairly good approximation to the SNR; see Appendix A for more details.

The value of the spin $a$ of the final black hole and also of the amplitude $\mathcal{A}$ will depend on the initial parameters of the system, as in Eq. (3.1). This dependence is very poorly understood at present. We expect the final black hole to be rapidly spinning since, as explained in Sec. III B, the total angular momentum of the binary at the end of the inspiral is $\sim 0.9 \mathrm{M}^{2}$ when the individual black holes are non-spinning [65], and the individual black hole spins can augment this. Moreover, the individual black holes may typically have been spun up to near maximal rotation by an accretion disk [91]. For definiteness, we somewhat arbitrarily take $a$ $=0.98$, which corresponds, from Eq. (3.17), to $Q=12$ and $f_{\mathrm{qnr}}=0.13 / M$. The final ringdown SNRs we obtain vary only weakly with our assumed value of $a$ [cf. Eq. (B14)], for fixed total energy radiated in the ringdown.

Although the value of the overall amplitude $\mathcal{A}$ is uncertain, we can estimate an upper bound on it for equal mass BBHs. Consider a Kerr black hole, distorted by an $l=m$
$=2$ perturbation such that the horizon's cross section is a rotating oval, rather than a circle. Quantify the distortion by computing the ratio of the polar circumference about the long axis of this oval to that about the short axis. Let $\mathcal{A}_{2}$ denote the perturbation amplitude such that this ratio of circumferences is $2: 1$. Clearly, the validity of linear perturbation theory must break down for amplitudes $\mathcal{A} \gtrsim \mathcal{A}_{2}$ (due to nonlinear couplings between the $l=m=2$ mode and other modes). At this 2:1 distortion ratio, the signal will not be very well approximated by just the $l=m=2$ mode. Therefore, $\mathcal{A}_{2}$ is a reasonable upper bound for the true amplitude $\mathcal{A}$.

In principle, we could calculate $\mathcal{A}_{2}$ by writing the spacetime metric as

$$
g_{a b}=g_{a b}^{\mathrm{KERR}}+\mathcal{A}_{2} h_{a b}^{\mathrm{QNR}}
$$

where $g_{a b}^{\mathrm{KERR}}$ is the Kerr metric and $h_{a b}^{\mathrm{QNR}}$ is the $l=m=2$ quasinormal mode whose asymptotic form at large $r$ is given by Eq. (3.15), and by calculating from this metric the ratio of circumferences [92]. For this paper, we used a much less sophisticated method to estimate $\mathcal{A}_{2}$. Using the quadrupole formula, we examined the radiation produced by a solid body that is distorted to this $2: 1$ circumference ratio, and obtained the estimate $\mathcal{A}_{2} \approx 0.4$ [93]. Setting our waveform amplitude $\mathcal{A}$ to this value yields an rms angle-averaged waveform $h=(0.4 / \sqrt{4 \pi})(M / r)=0.1(M / r)$ at the beginning of ringdown. From Eq. (3.18), the corresponding radiated energy is

$$
E_{\text {ringdown }} \approx \frac{1}{8} \mathcal{A}^{2} M^{2} f_{\text {qnr }} Q \approx 0.03 M .
$$

As mentioned in the Introduction, comparable ringdown radiation efficiencies of $\sim 3 \%$ have been seen in numerical simulations of the evolution of distorted, spinning black holes [66].

To summarize, our assumed values for the black hole spin parameter $a$ and for the amplitude $\mathcal{A}$ for equal-mass BBHs are

$$
\begin{aligned}
& a=0.98 \\
& \mathcal{A}=0.4 .
\end{aligned}
$$

These imply the values

$$
\begin{aligned}
f_{\mathrm{qnr}} & =\frac{0.13}{M}=1320 \mathrm{~Hz}\left(\frac{20 M_{\odot}}{M}\right) \\
Q & =12 \\
\epsilon_{\text {ringdown }} & =E_{\text {ringdown }} / M=0.03 .
\end{aligned}
$$

For non-equal-mass BBHs, we assume that $\epsilon_{\text {ringdown }}$ is reduced by the factor (1.5).

\section{E. Number of independent frequency bins for the merger phase}

In Sec. II A we showed that for any burst of gravitational waves, the band-pass filtering SNR is smaller than the matched filtering SNR by a factor of approximately 


$$
\sqrt{\mathcal{N}_{\text {bins }}}=\sqrt{2 T \Delta f}
$$

[cf. Eq. (2.13) and associated discussion]. In this section, we estimate $\mathcal{N}_{\text {bins }}$ for the merger gravitational waves, and hence determine the factor by which the SNR is degraded if bandpass filtering rather than matched filtering is used for merger wave searches.

First consider the bandwidth $\Delta f$. Our assumed bandwidth for the merger signal is $\Delta f=f_{\text {qnr }}-f_{\text {merge }} \approx f_{\text {qnr }}$ [ since $f_{\text {merge }}$ $\ll f_{\text {qnr }}$; cf. Eqs. (3.3) and (3.4)]. We cannot, however, be completely confident that all signal power in the merger will lie at frequencies below $f_{\mathrm{qnr}}$, and so a more appropriate choice might be $\Delta f \sim 2 f_{\mathrm{qnr}}$. Also the quasi-normal ringing frequency $f_{\text {qnr }}$ depends on the dimensionless spin parameter $a$ of the final black hole as given by Eq. (3.17). Choosing the highest possible value, $f_{\mathrm{qnr}}=[1 /(2 \pi M)]$, yields

$$
\Delta f \sim \frac{1}{\pi M} .
$$

Turn, now, to the effective duration $T$ of the merger, defined by Eq. (2.14). We expect that $T$ will vary considerably from event to event, depending on the black hole parameters. To get a feeling for the range possible values of $T$, consider first the type of coalescence described in Sec. III B, with both black holes nearly maximally spinning with spins and orbital angular momentum aligned. In this favorable case, recall that the binary has to shed an excess angular momentum of about $0.4 M$ during the merger in order to settle down its final Kerr state. Thus, the two black holes might well be centrifugally hung-up, orbiting for many cycles before their event horizons merge, so that the duration of the merger might be quite long. By contrast, when two non-spinning black holes merge, there is probably no excess angular momentum that must be shed after the orbital dynamical instability, and so the merger might be fairly quick. (In such a case, the ringdown waves might carry most of the emitted energy.)

To estimate $T$ in the angular-momentum-excess scenario, assume that the luminosity $d E / d t$ during the merger is about the same as the luminosity at the start of the ringdown, $2 \epsilon_{\text {ringdown }} M / \tau$. Since the total energy radiated in the merger is $\epsilon_{\text {merger }} M$, we find

$$
T \approx \frac{1}{2} \frac{\epsilon_{\text {merger }}}{\epsilon_{\text {ringdown }}} \tau
$$

Clearly this estimate will become invalid for high values of $\tau$ $(a \rightarrow 1)$; in that limit, the high quality factor of the QNR mode causes a low QNR luminosity, whereas there is no reason for the merger luminosity to be comparably low. Nevertheless, we insert our assumed parameter values (3.12), (3.22), and (3.21) into Eqs. (3.17) and (3.25) and find $T$ $\sim 50 M$. Combining this with Eqs. (2.13) and (3.24) yields

$$
\sqrt{\mathcal{N}_{\text {bins }}} \sim \sqrt{30} \sim 5
$$

For inspiraling Schwarzschild black holes, on the other hand, $T$ may not be much larger than a single QNR damping time: $T \approx \tau \approx 10 M$ (assuming $a=0.5$ say), yielding $\sqrt{\mathcal{N}_{\text {bins }}} \approx \sqrt{6}$.
The factor $\sqrt{\mathcal{N}_{\text {bins }}}$ is thus likely to lie in the range 2 $\lesssim \sqrt{\mathcal{N}_{\text {bins }}} \leq 5$. We adopt the estimate $\sqrt{\mathcal{N}_{\text {bins }}}=4$ in Sec. VI B to estimate the reduction in SNR resulting from using bandpass filtering instead of matched filtering. We use the conservatively large value $\mathcal{N}_{\text {bins }}=60$ in Sec. VI B to estimate detection thresholds for noise-monitoring searches for signals.

\section{INTERFEROMETER NOISE CURVES}

In this section we describe our piecewise power law, analytic approximation to the noise curves for initial LIGO interferometers, advanced LIGO interferometers, and the LISA interferometer. We express our model in terms of the dimensionless quantity $h_{\text {rms }}(f) \equiv \sqrt{f S_{h}(f)}$, where $S_{h}(f)$ is the one sided power spectral density of the interferometer noise [61]. Our model for the noise spectrum is

$$
h_{\mathrm{rms}}(f)= \begin{cases}\infty, & f<f_{s}, \\ h_{m}\left(\alpha f / f_{m}\right)^{-3 / 2}, & f_{s} \leqslant f<f_{m} / \alpha, \\ h_{m}, & f_{m} / \alpha \leqslant f<\alpha f_{m}, \\ h_{m}\left[f /\left(\alpha f_{m}\right)\right]^{3 / 2}, & \alpha f_{m}<f .\end{cases}
$$

The noise curve depends on four parameters: (i) A lower shutoff frequency $f_{s}$ below which the noise rapidly becomes very large and can be taken to be infinite. For ground-based interferometers, this low-frequency shutoff is due to seismic noise; for LISA, it is due to accelerometer noise (Ref. [7], p. 23). (ii) A frequency $f_{m}$, which is the location of the center of the flat portion of the spectrum. (iii) A dimensionless parameter $h_{m}$, which is the minimum value of $h_{\mathrm{rms}}(f)$. (iv) A dimensionless parameter $\alpha$ which determines the width of the flat portion of the noise curve. We approximate the noise curves by piecewise power laws in this way for calculational convenience.

For initial and advanced LIGO interferometers, we determined best-fit values of the parameters $f_{s}, f_{m}, h_{m}$ and $\alpha$ by fitting to the noise curves given in Ref. [3]. (Note that Fig. 7 of Ref. [3] is a factor of 3 too small from $\sim 10 \mathrm{~Hz}$ to $\sim 70 \mathrm{~Hz}$. This error does not appear in Fig. 10 of that reference [94].) The resulting parameter values are

$$
\left.\begin{array}{rl}
f_{s} & =40 \mathrm{~Hz} \\
f_{m} & =160 \mathrm{~Hz} \\
\alpha & =1.4 \\
h_{m} & =3.1 \times 10^{-22}
\end{array}\right\} \begin{aligned}
& \text { initial LIGO } \\
& \text { interferometer, }
\end{aligned}
$$

and

$$
\left.\begin{array}{rl}
f_{s} & =10 \mathrm{~Hz} \\
f_{m} & =68 \mathrm{~Hz} \\
\alpha & =1.6 \\
h_{m} & =1.4 \times 10^{-23}
\end{array}\right\} \begin{aligned}
& \text { advanced LIGO } \\
& \text { interferometer. }
\end{aligned}
$$

For ground-based interferometers, the $f^{-3 / 2}$ portion of our approximate formula (4.1) models the thermal suspension noise and the $f^{3 / 2}$ portion models the laser shot noise [97].

For the space-based LISA interferometer, we determined best-fit values of the parameters $f_{m}, h_{m}$ and $\alpha$ by fitting to 
the noise curve given in Ref. [10], and obtained the lower cutoff frequency $f_{s}$ from Ref. [7]. The resulting parameter values are

$$
\left.\begin{array}{rl}
f_{s} & =10^{-4} \mathrm{~Hz} \\
f_{m} & =3.7 \times 10^{-3} \mathrm{~Hz} \\
\alpha & =5.5 \\
h_{m} & =5.8 \times 10^{-22}
\end{array}\right\} \begin{aligned}
& \text { LISA } \\
& \text { interferometer. }
\end{aligned}
$$

Our piecewise power-law model is less accurate for LISA than for the LIGO interferometers, but it is still a fairly good approximation.

The sensitivity of LISA at the lower end of its frequency window may be degraded somewhat by a background of gravitational waves from white dwarf binaries [7]. We neglect this issue here as this white dwarf noise level is fairly uncertain (see Ref. [99] for a recent discussion).

\section{SIGNAL-TO-NOISE RATIOS}

In this section we calculate the angle-averaged SNRs for the three coalescence epochs (inspiral, merger, and ringdown) for initial LIGO interferometers, for advanced LIGO interferometers, and for LISA.

\section{A. Specific examples}

We start by rewriting the general formula (2.30) for the SNR in a more useful form. If we define the characteristic gravitational-wave amplitude

$$
h_{\text {char }}(f)^{2} \equiv \frac{2(1+z)^{2}}{\pi^{2} D(z)^{2}} \frac{d E}{d f}[(1+z) f],
$$

then from Sec. II C the SNR squared (2.7) for an optimally oriented source can be written as

$$
\rho_{\text {optimal orientation }}^{2}=\int d(\ln f) \frac{h_{\text {char }}(f)^{2}}{h_{\mathrm{rms}}(f)^{2}},
$$

where $h_{\text {rms }}(f)=\sqrt{f S_{h}(f)}$. From Eq. (2.30), the angleaveraged SNR squared is a factor of 5 smaller than the optimal value (5.2); so we can rewrite Eq. (2.30) as

$$
\left\langle\rho^{2}\right\rangle=\int d(\ln f) \frac{h_{\text {char }}(f)^{2}}{h_{n}(f)^{2}},
$$

where $h_{n}(f) \equiv \sqrt{5} h_{\mathrm{rms}}(f)$ is the rms noise appropriate for waves from random directions with random orientations [100]. Plotting $h_{\text {char }}(f)$ and $h_{n}(f)$ for various sources illustrates [from Eq. (5.3)] the possible SNR values and the distribution of SNR squared with frequency.

In Fig. 1, we show the rms noise amplitude $h_{n}(f)$ for our model (4.1) of the initial and advanced LIGO interferometer noise curves, together with the characteristic amplitude $h_{\text {char }}(f)$ for two different BBH coalescences: a coalescence of total mass $20 M_{\odot}$ at a distance of $D=200 \mathrm{Mpc}$ and a $30 M_{\odot}$ coalescence at redshift $z=1$. (We assume that the cosmological parameters are $\Omega_{0}=1$ and $H_{0}$ $=75 \mathrm{~km} \mathrm{~s}^{-1} \mathrm{Mpc}^{-1}$.) In each case, the sloped portion of the dashed $h_{\text {char }}$ line is the inspiral signal, the flat portion is our crude model of the merger, and the separate dotted portion is

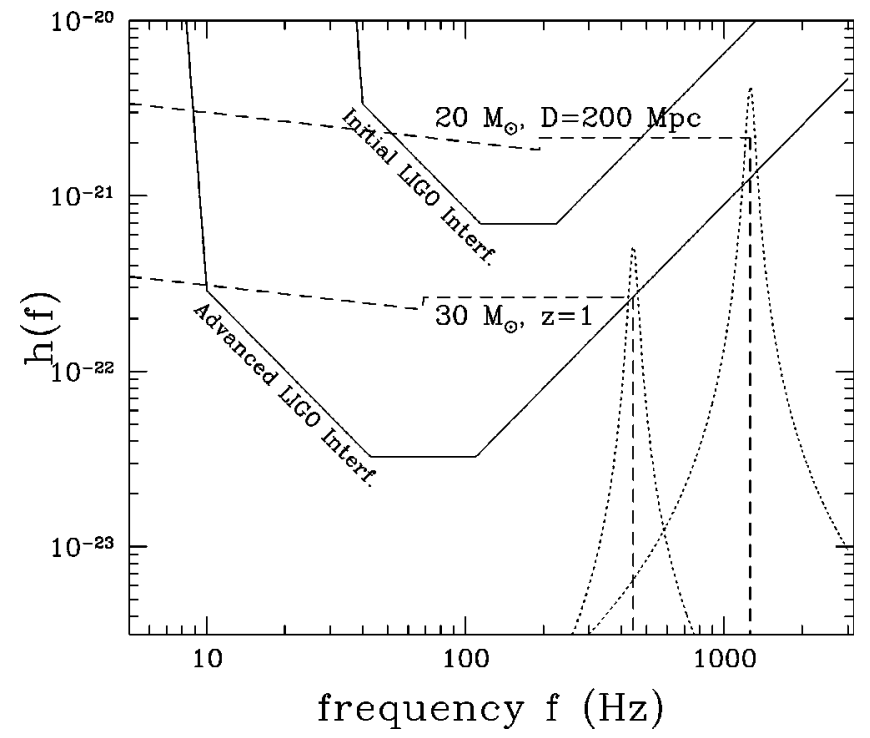

FIG. 1. An illustration of the relative magnitudes of our estimates of the inspiral, merger and ringdown energy spectra in two different cases. The solid lines are the rms noise amplitudes $h_{n}(f) \equiv \sqrt{5 f S_{h}(f)}$ for our assumed model (4.1) of the LIGO initial and advanced interferometer noise spectra. The dashed and dotted lines show the characteristic amplitude $h_{\text {char }}(f) \propto \sqrt{d E / d f}$ of the waves, defined by Eq. (5.1). The definition of $h_{\text {char }}$ is such that the signal-to-noise ratio squared for a randomly oriented source is given by $(S / N)^{2}=\int d(\ln f)\left[h_{\text {char }}(f) / h_{n}(f)\right]^{2}$. The upper dashed and dotted lines correspond to a binary of two $10 M_{\odot}$ black holes at a distance of $D=200 \mathrm{Mpc}$. The sloped portion of the dashed line is the inspiral, which gives an SNR for the initial (advanced) interferometer noise curve of 2.6 (84). The flat portion is our crude model of the merger, which gives an SNR of 2.1 (16). The dotted line is our estimate of the ringdown, which gives an SNR of $0.1(0.86)$. The lower dashed and dotted lines correspond to a binary of two $15 M_{\odot}$ black holes at redshift $z=1$ (or at a luminosity distance of $D=4.6 \mathrm{Gpc}$; the cosmological parameters $\Omega_{0}=1$ and $H_{0}$ $=75 \mathrm{~km} \mathrm{~s}^{-1} \mathrm{Mpc}^{-1}$ were assumed). In this case the inspiral, merger and ringdown SNRs for the initial (advanced) interferometers are $0.08,0.42$, and $0.07(6.6,7.2$, and 0.5$)$ respectively. Black hole binaries with constituents this massive will be visible to great distances, making them a possibly important source, depending on the very uncertain event rate. The SNR from the merger is enhanced for these massive distant sources in part because the combination of cosmological redshift and lower intrinsic frequency brings the merger waves down to lower frequencies where the interferometer noise is smaller.

the ringdown. Note that the ringdown and merger overlap in the frequency domain since (as we have defined them) they are disjoint in the time domain, while the inspiral and merger are approximately disjoint in both the frequency and time domains (Sec. III A above).

In both cases, $20 M_{\odot}$ and $30 M_{\odot}$, the waves' characteristic amplitude $h_{\text {char }}(f)$ is rather larger than $h_{n}(f)$ for most of the merger spectrum for the advanced interferometers, indicating the detectability of the merger waveform when matched filtering can be used. In particular, note that the waves should be quite visible to the advanced interferometers for the $30 M_{\odot}$ binary even though it is at a cosmological distance. Even if such binaries are rare, they are visible to such great distances that they may be an important and in- 


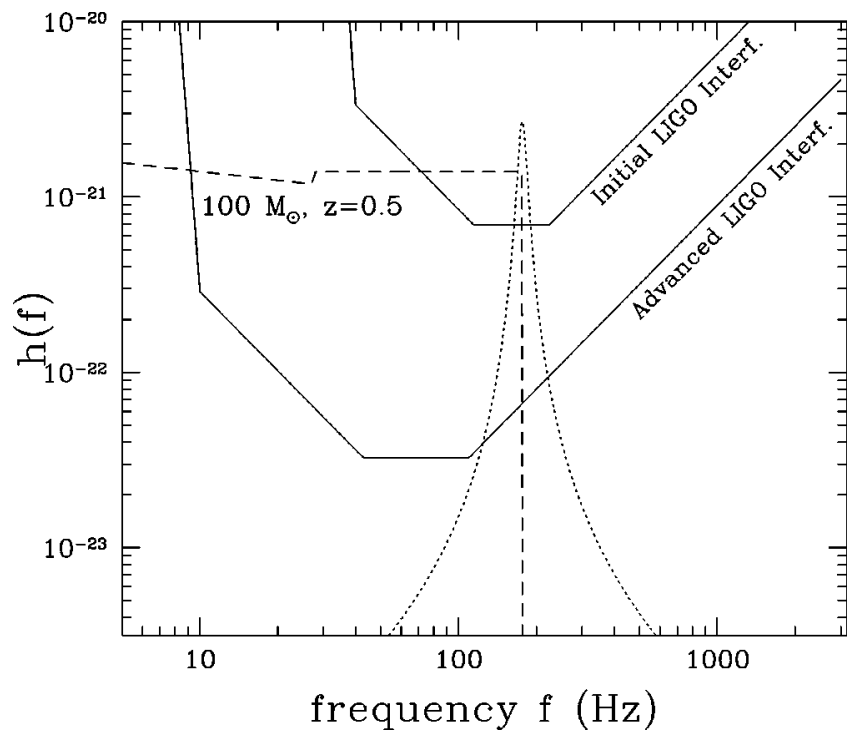

FIG. 2. A merger of a binary consisting of two $50 M_{\odot}$ black holes at redshift $z=0.5$, together with the rms noise amplitudes $h_{n}(f)$ for both the initial and advanced interferometer noise curves for LIGO (see caption of Fig. 1). The SNRs for the inspiral, merger, and ringdown stages are about $0,1.7$ and 1.0 respectively for the initial interferometer noise level, and about 11,52 and 11 respectively for advanced interferometers.

teresting source. Cosmological binaries have an enhanced SNR in part because the cosmological redshift moves their frequency spectrum down closer to LIGO's optimal band.

Figure 1 also shows that, of these two example BBH coalescences, only the nearby one at a distance of $D$ $=200 \mathrm{Mpc}$ would be detectable by the initial interferometers. As discussed in the Introduction, such coalescences may yield an interesting event rate for the initial interferometers.

A qualitatively different, possibly important type of source for the initial LIGO interferometers (and also for the advanced interferometers) is the coalescence of black hole binaries with masses of order $100 M_{\odot}$, as we have discussed in the Introduction. In Fig. 2 we show the characteristic amplitude $h_{\text {char }}(f)$ for a hypothetical BBH coalescence of total mass $100 M_{\odot}$ at redshift $z=0.5$, corresponding to a luminosity distance of $D=2.2 \mathrm{Gpc}$. Note in particular that the initial LIGO interferometer noise curve has best sensitivity near $200 \mathrm{~Hz}$ just where the (redshifted) ringdown frequency is located. We discuss further in Sec. VI the range of initial LIGO interferometers for this type of source.

Turn, now, to the detection of supermassive BBH signals by the space-based detector LISA [5,6]. LISA can study $\mathrm{BBH}$ mergers with far higher accuracy and resolution than the ground-based interferometers, because the SNR values are typically much higher $\left(\gtrsim 10^{3}\right)$. When calculating inspiral SNRs for LISA, it is necessary to restrict the integral over frequency in Eq. (2.30) to a domain that corresponds to, say, $1 \mathrm{yr}$ of observation-some binaries require hundreds of years to pass through LISA's band. See Appendix B for details.

Figure 3 shows our approximate model [Eqs. (4.1) and (4.4)] of LISA's projected noise spectrum, together with the gravitational-wave amplitude $h_{\text {char }}(f)$ for the inspiral, merger and ringdown stages of two different $\mathrm{BBH}$ coalescences: a $\mathrm{BBH}$ of total mass $10^{6} M_{\odot}$ at redshift $z=5$ and a

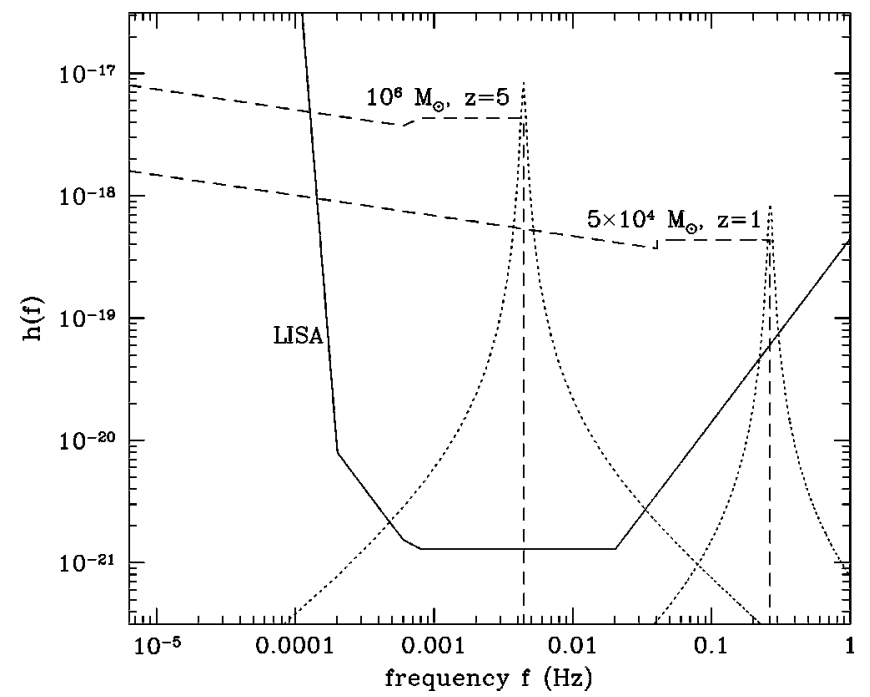

FIG. 3. The noise spectrum $h_{n}(f)$ of the space-based detector LISA, together with the characteristic amplitudes $h_{\text {char }}$ of two equalmass BBH coalescences (see caption of Fig. 1). The first is a binary of total mass $10^{6} M_{\odot}$ at redshift $z=5$. The inspiral signal of this binary enters the LISA waveband at $f \simeq 10^{-4} \mathrm{~Hz}$ about 1 week before the final merger; the SNRs from inspiral, merger and ringdown are about 1800, 4600 and 1700 respectively. The second is a binary of total mass $5 \times 10^{4} M_{\odot}$ at redshift $z=1$, which enters the LISA waveband about 20 years before the final merger. For this binary an SNR of approximately 900 would be obtained for the last year of inspiral (from $f \simeq 1.6 \times 10^{-4} \mathrm{~Hz}$ to $f \simeq 4 \times 10^{-2} \mathrm{~Hz}$ ). The SNRs from the merger and ringdown would be about 70 and 4 .

BBH of total mass $5 \times 10^{4} M_{\odot}$ at redshift $z=1$. The $10^{6} M_{\odot}$ $\mathrm{BBH}$ enters the LISA waveband at $f=f_{s} \simeq 10^{-4} \mathrm{~Hz}$ roughly 1 week before the final merger. The SNRs obtained in this case from the inspiral, merger and ringdown signals are approximately 1800,4600 and 1700 respectively. The $5 \times 10^{4} M_{\odot}$ BBH enters the LISA waveband about $20 \mathrm{yr}$ before the final merger. The SNR obtained from the last year of the inspiral signal, from $f \simeq 1.6 \times 10^{-4} \mathrm{~Hz}$ to $f \simeq$ $4 \times 10^{-2} \mathrm{~Hz}$ is approximately 900 , while the merger and ringdown SNRs are about 70 and 4 respectively.

\section{B. General signal-to-noise ratio results}

We now turn from these specific examples to the dependence of the SNR values on the mass of and distance to the binary in general. In Appendix B we obtain analytic formulas for the SNR values for the three phases of BBH coalescences and for the various interferometers. In this section we plot the results for equal-mass BBHs, which are shown in Figs. 4, 5 and 6. The inspiral and merger curves in these figures (except for the LISA inspiral curves; see Appendix B) are obtained from Eqs. (B4) and (B10) of Appendix B, while the ringdown curves are obtained by numerically integrating Eq. (3.19) in Eq. (2.30).

The SNR values for the initial LIGO interferometers are shown in Fig. 4. This figure shows that an important source for the initial LIGO interferometers may be the coalescences of binary black holes with total masses of the order of several hundred solar masses. These would be visible out to almost $1 \mathrm{Gpc}$. For such sources, the inspiral portion of the signal would not be detectable, and one would need to search 


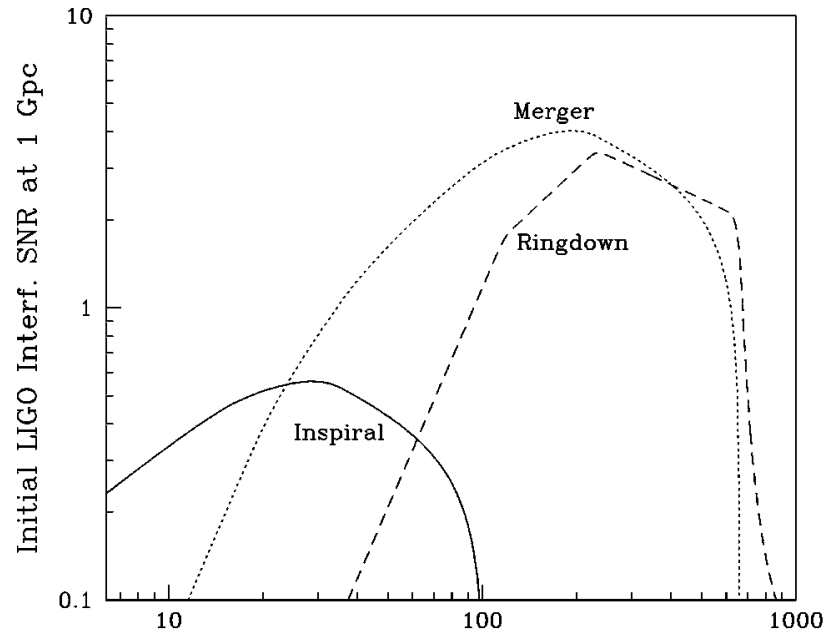

$(1+\mathrm{z}) \mathrm{M}$ measured by Initial LIGo Interferometer $\left(\mathrm{M}_{\odot}\right)$

FIG. 4. The SNR for equal-mass BBH coalescences detected by LIGO initial interferometers, assuming matched filtering, as a function of the redshifted mass $(1+z) M$ of the final black hole, at a luminosity distance of $D=1 \mathrm{Gpc}$. For fixed redshifted mass, the SNR values are inversely proportional $D$. The solid, dotted, and dashed curves are the SNR values from the inspiral, merger and ringdown respectively. For non-equal-mass binaries, the inspiral SNRs will be reduced by the factor $\sim \sqrt{4 \mu / M}$, while the merger and ringdown SNRs will be reduced by $\sim 4 \mu / M$; thus the inspiral will be enhanced relative to the merger and ringdown. This plot indicates that $\mathrm{BBH}$ coalescences of systems with masses of the order of several hundred solar masses may be an important source for the initial LIGO interferometers. These events would be visible to almost $1 \mathrm{Gpc}$. For such sources, the inspiral would not be detectable, and the waves would have to be detected using either the ringdown or the merger.

for the ringdown or merger to detect the waves. See Sec. VI for further discussion. The event rate for such high mass BBHs is very uncertain; see Ref. [17] for a possible formation scenario. Intermediate mass BBHs with $\mu \ll M$ (e.g., $\left.m_{1}=10 M_{\odot}, m_{2}=500 M_{\odot}\right)$ are presumably much more common than the intermediate mass BBHs with $\mu \sim M$ discussed above. The SNRs for such mixed binaries will be much lower, however. As seen in Appendix A, the merger and ringdown SNRs scale as $(\mu / M)^{2}$, while the inspiral ringdown scales as $\mu / M$. (The scaling difference arises because the inspiral duration scales as $1 / \mu$, whereas the merger and ringdown durations are approximately independent of $\mu$.)

Figure 4 also shows that the inspiral of BBH mergers with $M \lesssim 30 M_{\odot}$ should be visible to about $200 \mathrm{Mpc}$ (the SNR detection threshold is about 5 [44]). The ground-based interferometers will, over a period of years, gradually be improved from the initial sensitivity levels to advanced sensitivity levels [3]. Roughly halfway between the initial and advanced interferometers, the range of the detector system for $M \lesssim 30 M_{\odot} \mathrm{BBHs}$ will be $\sim 1 \mathrm{Gpc}$. If the $\mathrm{BBH}$ birthrate is as large as was discussed in the Introduction, they should be detected early in the gradual process of interferometer improvement.

Figure 5 shows the SNR values for the advanced LIGO interferometers. It can be seen that for advanced LIGO interferometers, equal-mass $\mathrm{BBH}$ inspirals will be visible out to $z \sim 1 / 2$ for the entire range of masses $10 M_{\odot} \lesssim(1+z) M$

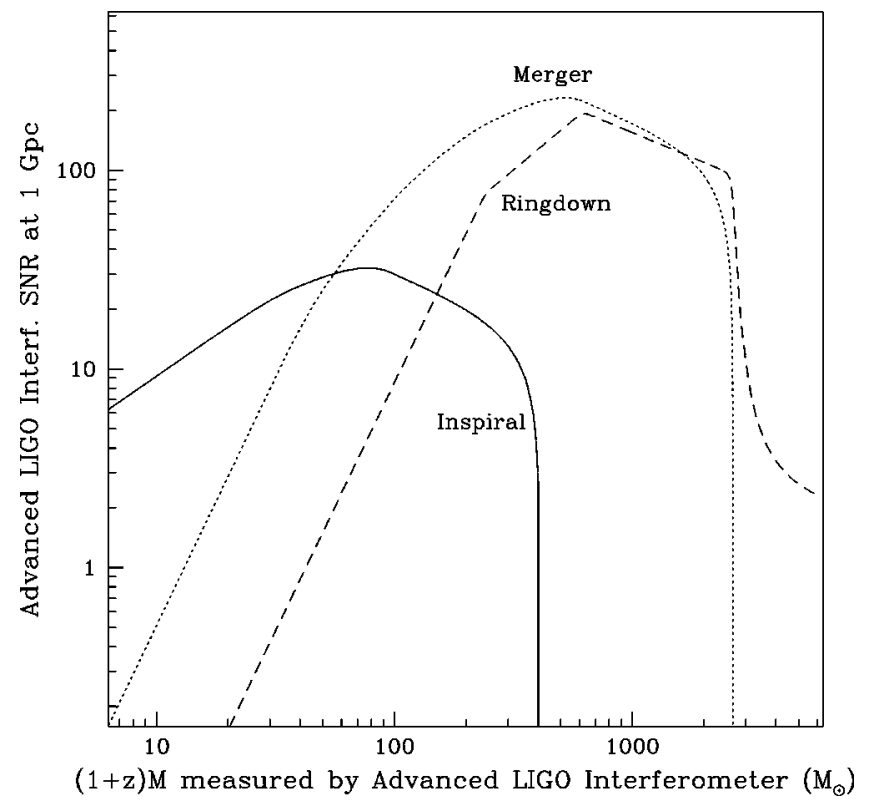

FIG. 5. The SNR values for advanced LIGO interferometers for the inspiral (solid line), merger (dotted line) and ringdown (dashed line) phases of equal-mass BBH coalescences at a luminosity distance of $D=1 \mathrm{Gpc}$; see the caption of Fig. 4. For values of the redshifted final mass lower than $\sim 60 M_{\odot}$ the inspiral SNR is largest, while for larger BBH systems the merger and/or ringdown portions of the signal dominate.

$\$ 300 M_{\odot}$. Thus, there is likely to be an interesting event rate. Indeed, the SNRs will be high enough even for rather large distances that it should be possible to extract each binary's parameters with reasonable accuracy [10]. By contrast, the ringdown SNR is fairly small except for the largest mass systems. For very massive binaries or binaries that are closer than $1 \mathrm{Gpc}$, advanced interferometers may measure fairly large ringdown SNRs, which would allow fairly good estimates of the mass and spin of the final black hole $[15,16]$.

Figure 6 shows the SNR values obtainable from the three phases of BBH coalescences by LISA: the last year of inspiral, the merger and the ringdown. We also show the SNR value obtainable from $1 \mathrm{yr}$ of integration of the inspiral signal $100 \mathrm{yr}$ before the merger, and a similar curve for $1000 \mathrm{yr}$ before the merger. This figure shows that LISA will be able to perform very high accuracy measurements of BBH mergers (SNR values $\gtrsim 10^{3}$ ) essentially throughout the observable Universe $(z \lesssim 10)$ in the mass range $10^{6} M_{\odot} \lesssim M \lesssim 10^{9} M_{\odot}$. As discussed in the Introduction, there is a good chance there will be an interesting event rate. The SNR curves in Fig. 6 for measurements 100 and 1000 yr before merger show that many inspiraling BBHs that are far from merger should be detectable by LISA as well. If the merger rate of SMBH binaries turns out to be about one per year throughout the observable Universe, then at any given time one would expect roughly $1000 \mathrm{SMBH}$ binaries to be $1000 \mathrm{yr}$ or less away from merger. LISA will be able to monitor the inspiral of such binaries (if they are of sufficiently low mass) with moderate to large SNR [10].

Finally, it should be noted that the relative magnitude of the merger and ringdown SNR values is somewhat uncertain. We have assumed a total radiated energy of $0.1 \mathrm{M}$ in the merger portion of the signal and $0.03 M$ in the ringdown 


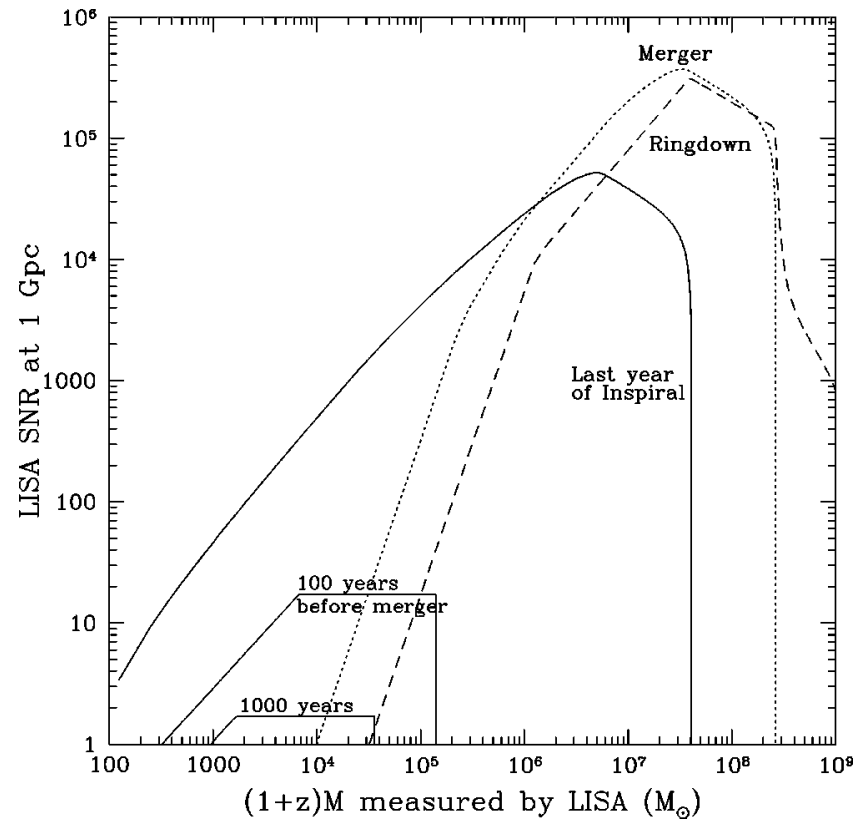

FIG. 6. The SNR for equal-mass BBH coalescences detected by LISA, assuming matched filtering, as a function of the redshifted mass $(1+z) M$ of the final black hole, at a luminosity distance of $D=1 \mathrm{Gpc}$. The dotted and dashed curves are the SNR values from the merger and ringdown, respectively. The upper solid curve is the SNR that would be obtained from measuring the last year of the inspiral. For $(1+z) M \leq 10^{6} M_{\odot}$, the last-year-inspiral SNR is largest; for larger BBH systems the merger and/or ringdown dominate. Also shown (lower solid curves) are the SNRs that would be obtained from $1 \mathrm{yr}$ of integration of the inspiral at 100 and $1000 \mathrm{yr}$ before the final merger. If the rate of SMBH coalescences within $z \lesssim$ (a few) is roughly one per year, then one would expect roughly one thousand SMBH binaries to be a $1000 \mathrm{yr}$ or less away from merger. This plot shows that LISA will be able to measure the inspiral of such binaries (provided they are of sufficiently low mass) with moderate to large SNR [10].

portion, a ratio of $3: 1$. It may turn out that in individual cases the ratio is as high as 10 or as low as $\lesssim 1$. It may even turn out to be the case that for many coalescences, the ringdown portion of the waveform carries most of the radiated energy of the combined merger and ringdown regime (depending possibly on the distribution of initial spins). Thus, the SNR values shown in Figs. 4, 5 and 6 should merely be taken as illustrative.

\section{IMPLICATIONS FOR DETECTABILITY OF THE GRAVITATIONAL-WAVE SIGNAL}

One of the reasons that coalescences of compact objects are such good sources for gravitational-wave detectors is that the inspiral is very predictable, so that matched filtering may be used for signal searches [1]. As we have discussed, matched filtering enhances the achievable inspiral SNR values by a factor of roughly $\sqrt{\mathcal{N}_{\text {cyc }}}$, where $\mathcal{N}_{\text {cyc }}$ is the number of cycles of the waveform in the frequency band of the detectors. For neutron-star-neutron-star (NS-NS) coalescences, $\mathcal{N}_{\text {cyc }}$ will be on the order of several thousand, while for low mass $\left(M \lesssim 50 M_{\odot}\right) \mathrm{BBH}$ coalescences it will be on the order of several hundred [44]. Thus, for NS-NS coalescences and for low mass $\mathrm{BBH}$ coalescences, the inspiral will be used to detect the entire waveform. In these cases, it is not necessary to search for the merger and ringdown portions of the waveform, since it will be known roughly where in the interferometer data stream they are expected to lie.

For larger mass BBHs, however, our results show that the merger and ringdown SNRs can be larger than the inspiral SNRs. For equal-mass BBHs, this will occur whenever (1 $+z) M \gtrsim 30 M_{\odot}$ for the initial LIGO interferometers, and whenever $(1+z) M \gtrsim 60 M_{\odot}$ for the advanced LIGO interferometers. Indeed, the inspiral SNR completely shuts off for large enough $(1+z) M$, as can be seen from Figs. 4 and 5 . Admittedly, $\mathrm{BBH}$ binaries of total mass $\gg 20 M_{\odot}$ may well be very much more rare than $\mathrm{BBH}$ binaries of $\sim 20 M_{\odot}$; however, they will be visible to such great distances that there may be an interesting event rate. Moreover, for the initial LIGO interferometers, the mass scale $\sim 30 M_{\odot}$ at which the inspiral SNR becomes much smaller than the merger and ringdown SNRs is not terribly high.

In such high mass cases for which the merger and inspiral SNRs exceed the inspiral SNR, it will be necessary to perform a search for the merger and/or ringdown portions of the signal, independently of any searches for inspiral signals, in order that all possible events be detected. If one seeks to detect the waves merely by optimal filtering for the inspiral waveform, some fraction of the events will be missed which otherwise might have been detectable. In fact, it may very well turn out that merger signals from $\mathrm{BBH}$ coalescences could be the dominant source for the initial LIGO interferometers.

One might imagine that the gravitational waves would generally be easier to detect by searching for the merger signal than for the ringdown, since we have estimated that the SNR values for the merger phase are typically a factor of a few larger than those for the ringdown (cf. Figs. 4 and 5). There are several factors that complicate this conclusion, however. On the one hand, the ringdown's waveform shape is better understood, which makes it easier to produce search templates and hence easier to detect the signal. On the other hand, the ratio between the merger and ringdown SNRs is really quite uncertain, as discussed in Sec. V B, and so it is plausible that the merger SNR will be larger than we have indicated relative to the ringdown SNR. In any case, the ratio between merger and ringdown SNRs will presumably vary a lot from event to event. Thus, it would seem that searches will be necessary for both types of signal in the data stream, at least for the mass range in which the ringdown SNR is expected to exceed the inspiral SNR. [From Sec. V we estimate this mass range to be $(1+z) M \gtrsim 200 M_{\odot}$ for the advanced interferometers, and $(1+z) M \gtrsim 60 M_{\odot}$ for the initial interferometers.]

We summarize the discussion of this subsection by displaying the optimum search strategies for various mass ranges for the three different interferometers. In each case below, the mass range marked merger refers to matched filtering searches for merger signals. If merger templates are available, then in the indicated mass ranges merger searches will probably be more successful than inspiral or ringdown searches; the question mark is a reminder that merger templates may not be available. 


$$
\left.\begin{array}{lll}
\text { inspiral } & : & 1 M_{\odot} \lesssim M \lesssim 60 M_{\odot} \\
\text { ringdown } & : & 60 M_{\odot} \lesssim M \lesssim 1000 M_{\odot} \\
\text { merger(?) } & : & 30 M_{\odot} \lesssim M \lesssim 1000 M_{\odot}
\end{array}\right\} \begin{aligned}
& \text { LIGO } \\
& \text { initial } \\
& \text { interf. }
\end{aligned}
$$

$$
\left.\begin{array}{lll}
\text { inspiral } & : & 1 M_{\odot} \lesssim M \lesssim 200 M_{\odot} \\
\text { ringdown } & : & 200 M_{\odot} \lesssim M \lesssim 3000 M_{\odot} \\
\text { merger(?) } & : & 80 M_{\odot} \lesssim M \lesssim 3000 M_{\odot}
\end{array}\right\} \begin{aligned}
& \text { LIGO } \\
& \text { advanced } \\
& \text { interf. }
\end{aligned}
$$

$$
\left.\begin{array}{lll}
\text { inspiral } & : & 10^{3} M_{\odot} \leq M \leq 10^{7} M_{\odot} \\
\text { ringdown } & : & 10^{7} M_{\odot} \leq M \leq 10^{9} M_{\odot} \\
\text { merger(?) } & : & 2 \times 10^{6} M_{\odot} \leq M \leq 10^{9} M_{\odot}
\end{array}\right\} \text { LISA }
$$

\section{A. Detectability of high mass black-hole coalescences via the ringdown signal}

Consider first the search for ringdown signals. In this case, since the shape of the signal is known up to several unknown parameters, it will be feasible to implement a matched filtering search. The number $\mathcal{N}_{\text {templates }}$ of required templates [72] can be estimated by combining the formalism developed by Owen [89] and the results of Echeverria and Finn on the expected measurement accuracy of the ringdown frequency and damping time $[15,16]$. Using Eqs. (4.15) of Ref. [16] and Eqs. (2.23) and (2.28) of Ref. [89] we find that the metric defined by Owen on the space of parameters is given by $[101]$

$$
d s^{2}=\frac{1}{8 Q^{2}} d Q^{2}+\frac{Q^{2}}{2 f_{\mathrm{qnr}}^{2}} d f_{\mathrm{qnr}}^{2},
$$

where $Q$ is the quality factor. The formula (6.4) for the Owen metric is valid only in the high $Q$ limit; it has corrections of order $1 / Q^{2}$. Moreover, the formula is also only valid when the noise spectrum $S_{h}(f)$ does not vary significantly within the resonance bandwidth $\Delta f \sim f_{\text {qnr }} / Q$. Therefore estimates obtained from Eq. (6.4) for the number of template shapes required for ringdown searches will only be accurate to within factors of order unity; this is adequate for our purposes.

Using Eq. (2.16) of Ref. [89] we find that that the number of required templates is approximately

$$
\mathcal{N}_{\text {templates }} \approx \frac{1}{8} Q_{\max }(1-M M)^{-1} \ln \left[\frac{M_{\max }}{M_{\min }}\right],
$$

where $Q_{\max }, M_{\min }$ and $M_{\max }$ are the extremal values of the quality factor and of the black hole mass that define the range of signal searches. The quantity $M M$ in the formula (6.5) is the minimal match parameter introduced by Owen. A lattice of templates with minimal match $M M$ will have an event detection rate smaller than the ideal rate (achieved with an infinitely dense template grid) by the factor $(M M)^{3}$ [89]. We assume $M M=0.97$ as in Ref. [89], corresponding to a $10 \%$ event rate loss, and take $Q_{\max }=100$ [which by Eq. (3.17) corresponds to $\left.1-a \simeq 10^{-4}\right]$. For the initial and ad- vanced LIGO interferometers, the mass range to be searched corresponds to roughly $M_{\min } \simeq 1 M_{\odot}$ and $M_{\max }=5000 M_{\odot}$, yielding

$$
\mathcal{N}_{\text {templates }} \lesssim 4000 \text {. }
$$

This is a rather small number of templates compared to the number expected to be necessary for inspiral searches [89], and so a ringdown search should be fairly easy to implement. A similarly small number of required template shapes $\left(\mathcal{N}_{\text {templates }} \lesssim 6000\right)$ is obtained for LISA assuming $M_{\text {min }}$ $\sim 10^{3} M_{\odot}$ and $M_{\max } \sim 10^{9} M_{\odot}$.

We next discuss the distance to which $\mathrm{BBH}$ mergers should be detectable via their ringdown signals. As explained in Sec. II C, an estimate of the appropriate SNR threshold for detection using one interferometer is [102]

$$
\rho_{\text {threshold }} \approx \sqrt{2 \ln \left[\mathcal{N}_{\text {templates }} T /(\epsilon \Delta t)\right]}
$$

where $T$ is the observation time, $\Delta t$ is the sampling time and $\epsilon=10^{-3}$ is as defined in Sec. II B. In fact coincidencing between the 4 different interferometers in the LIGO-VIRGO network will be carried out, in order to increase detection reliability and combat non-Gaussian noise (see Sec. II A). If the noise were exactly Gaussian, the appropriate detection criterion would be to demand that

$$
\sum_{j} \rho_{j}^{2} \geqslant \rho_{\text {threshold }}^{2}
$$

where the sum is over the different SNRs obtained in each interferometer. In order to combat non-Gaussian noise, the detection criterion will be modified to require approximately equal SNRs in each interferometer:

$$
\rho_{j} \geqslant \rho_{\text {threshold }} / \sqrt{2} \text { for all } j \text {. }
$$

We have chosen a factor of $\sqrt{2}$ here to be conservative; it corresponds to combining the outputs of just two interferometers (say, the two LIGO $4 \mathrm{~km}$ interferometers) instead of four interferometers.

Taking $T=10^{7} \mathrm{~s}$ and $\Delta t=1 \mathrm{~ms}$ yields the estimate $\rho_{\text {threshold }} / \sqrt{2} \approx 6.0$ for the initial and advanced LIGO interferometers. Therefore, from Fig. 4, we see that the initial LIGO interferometers should be able to see ringdowns from equalmass $\mathrm{BBHs}$ in the mass range $100 M_{\odot} \lesssim M \lesssim 700 M_{\odot}$ out to about $200 \mathrm{Mpc}$, if the radiation efficiency $\epsilon_{\text {ringdown }}$ is as large as we have estimated. The advanced LIGO interferometers, by contrast, should see ringdowns in the mass range $200 M_{\odot} \lesssim(1+z) M \lesssim 3000 M_{\odot}$ out to $z \sim 1$ (from Fig. 5). For non-equal-mass $\mathrm{BBHs}$, these distances are reduced roughly by the factor $\sim(4 \mu / M)$.

For LISA, the detection threshold is given by Eq. (6.7). Although LISA does incorporate several partially independent interferometers, we have used the noise spectrum (4.4) which is the effective noise spectrum that applies to the LISA detector as a whole [7]. Thus it is consistent to treat LISA as one interferometer. Taking $T=10^{7} \mathrm{~s}$ and $\Delta t=1 \mathrm{~s}$, and using the value $\mathcal{N}_{\text {shapes }}=6000$ estimated above yields $\rho_{\text {threshold }} \approx 7.5$. Hence, from Fig. 6 , LISA should see ringdowns in the mass range $10^{6} M_{\odot} \lesssim(1+z) M \lesssim 3 \times 10^{8} M_{\odot}$ out to $z \gtrsim 100$. 


\section{B. Detectability of high mass black hole coalescences via the merger signal}

We next discuss the feasibility of searches for the merger signal. As we have explained, this will be most necessary when the merger SNR is larger than both the inspiral and ringdown SNRs by factors of a few (since the fractional loss in event detection rate, if searches for the merger signal are not carried out, is the cube of the ratio of the SNR values).

Consider first the ideal situation in which theoretical template waveforms are available, so that matched filtering can be used in searches. From Figs. 4 and 5 it can be seen that the merger SNR values are larger than the inspiral and ringdown values by a factor of up to $\sim 4$, in the mass ranges $30 M_{\odot} \lesssim M \lesssim 200 M_{\odot}$ for initial LIGO interferometers and $100 M_{\odot} \lesssim M \lesssim 400 M_{\odot}$ for advanced LIGO interferometers. More precisely, in this mass range,

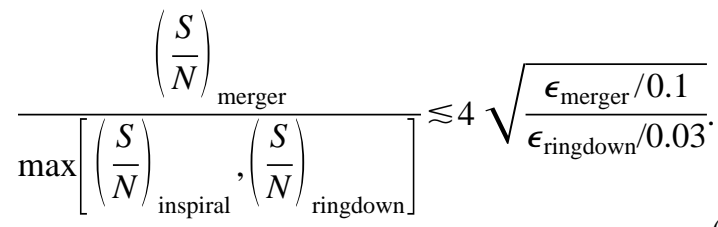

The detection threshold for merger searches should be approximately the same as that for inspiral and merger searches, if the number of template shapes $\mathcal{N}_{\text {shapes }}$ is not too large (see further discussion below). Therefore, the gain in event rate over inspiral and ringdown searches should vary between 1 and about $4^{3}=64$, depending on the mass of the system, if our estimates of $\epsilon_{\text {merger }}$ and $\epsilon_{\text {ringdown }}$ are reasonable. The large possible gain in event rate clearly demonstrates the importance of merger searches [103].

Note, however, that it is not clear how feasible it will be to produce a set of numerically generated templates that is complete enough to be used to successfully implement an optimal filtering search. There may be a very large number of distinct waveform shapes, each of which will require extensive numerical computations. If both black holes are spinning rapidly, the waveforms could depend in significant and nontrivial ways on 6 distinct angular parameters, suggesting that the number of distinct shapes could be very large.

Next, consider the situation in which merger templates are unavailable. Consider first band-pass filtering searches. From the estimate $\sqrt{\mathcal{N}_{\text {bins }}}=4$ of Sec. III E, combined with Eq. (2.15), we see that the merger SNR in a band-pass filtering search is reduced by a factor of 4 from the values presented in Appendix B and Figs. 4, 5, and 6. By Eq. (6.10), the achievable band-pass filtering merger SNR is likely to be essentially no larger than the inspiral and ringdown SNRs.

Noise monitoring searches for the merger will be more efficient than band-pass filtering searches, approaching the effectiveness of matched filtering searches. [By contrast, noise-monitoring searches for inspiral waves would perform very badly, since $\mathcal{N}_{\text {bins }}$ is much larger $(\gtrsim 1000)$ for inspiral waves than it is for merger waves $(\lesssim 60)]$. The eventdetection rate from noise-monitoring is a factor of

$$
\mathcal{R}=\left(\frac{\rho_{*}}{\rho_{\text {threshold }}}\right)^{3}
$$

lower than the event rate from matched filtering. Here $\rho_{*}$ is the noise-monitoring detection threshold, given by Eqs. (2.27) and (2.28) as a function of the parameters $\epsilon, \mathcal{N}_{\text {start-times }}$ and $\mathcal{N}_{\text {bins }}$, and $\rho_{\text {threshold }}$ is the matched filtering threshold, given by Eq. (2.8) as a function of the parameters $\mathcal{N}_{\text {shapes }}$ and $\mathcal{N}_{\text {start-times }}$. As discussed in Sec. II B, the calculation we have given of the threshold $\rho_{*}$ assumes Gaussian noise behavior; the possible residual non-Gaussianity of real data even after coincidencing between detectors may degrade the effectiveness of noise-monitoring.

We now estimate the loss factor in event rate $\mathcal{R}$. To obtain the most pessimistic estimate, we use the following assumptions: (i) The number of template shapes in the matched filtering search is $\mathcal{N}_{\text {shapes }}=1$. A realistic larger number would yield a smaller $\mathcal{R}$. (ii) The number of frequency bins is $\mathcal{N}_{\text {bins }}=60$, twice the upper limit estimated in Sec. III E [104]. (iii) The number of starting times in the data stream is $\mathcal{N}_{\text {start-times }}=10^{8}$, corresponding to a sampling time of $0.1 \mathrm{~s}$ in a data set of one-third of a year. Such a large sampling time (and small $\mathcal{N}_{\text {start-times }}$ ) would only be appropriate for the largest BBHs; more realistic sampling times will be smaller. Larger values of $\mathcal{N}_{\text {start-times }}$ give smaller values of $\mathcal{R}$. (iv) The parameter $\epsilon$ in Eqs. (2.27) and (2.8) is $\epsilon=10^{-3}$. With these assumptions we obtain $\rho_{\text {threshold }}=6.8, \rho_{*}=10.3$; the resulting loss factor is

$$
\mathcal{R}=3.5
$$

Hence, noise-monitoring searches should only be a factor of at most $\sim 4$, and more typically $\sim 2$, worse than matched filtering searches.

The above discussion assumed that $\mathcal{N}_{\text {shapes }}$ is small. As the number $\mathcal{N}_{\text {shapes }}$ increases, the advantage of matched filtering searches decreases; at some number $\mathcal{N}_{\text {shapes,max }}$, matched filtering and noise monitoring perform about equally well. In Ref. [73] we show that [105]

$$
\ln \left(\mathcal{N}_{\text {shapes, } \max }\right) \approx \frac{1}{2} \mathcal{N}_{\text {bins }} \ln \left(1+\rho^{2} / \mathcal{N}_{\text {bins }}\right)
$$

From Eqs. (6.12) and (2.27), the critical value of the number of shapes is $\sim 10^{13}$ for $\mathcal{N}_{\text {bins }}=60$, and $\sim 10^{7}$ for $\mathcal{N}_{\text {bins }}=20$, assuming $\mathcal{N}_{\text {start-times }}=10^{8}$.

The actual number of shapes, $\mathcal{N}_{\text {shapes }}$, will vary with the SNR level $\rho$. We can define an effective dimension $N_{d}$ of the manifold of signals by the equation

$$
\ln \left[\mathcal{N}_{\text {shapes }}(\rho)\right]=\frac{1}{2} N_{d}(\rho) \ln \left[1+\rho^{2} / N_{d}(\rho)\right] ;
$$

the parameter $N_{d}(\rho)$ is the dimension of the equivalent (linear) space of signals that has the same number of distinguishable wave shapes with SNR $\leqslant \rho$ as the true, curved, manifold of merger signals [57]. In Fig. 7, we show the gain factor $\mathcal{R}$ as a function of $\mathcal{N}_{\text {bins }}$ for the values $N_{d}=0,5$, and 10. The true value of $N_{d}$ is quite uncertain; at high SNR levels it could conceivably be as large as $\sim 10$.

Combining the gain factor of 64 discussed above with the loss factor $\mathcal{R}$, it follows that noise-monitoring searches for merger waves could increase the event rate-and hence the number of discovered BBHs-by a factor up to about 10 


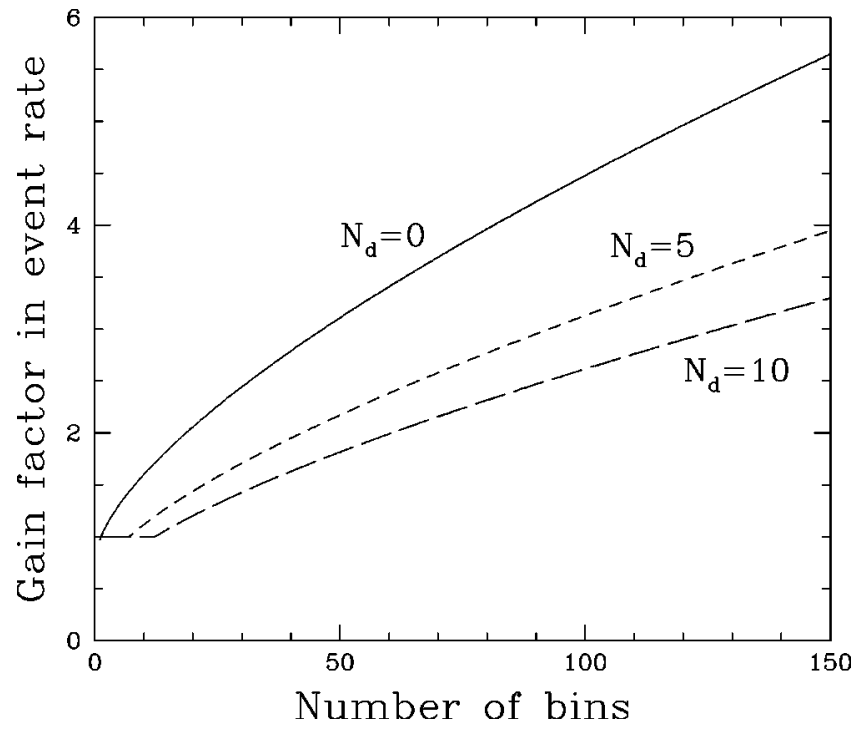

FIG. 7. The factor by which the event detection rate is increased when one uses matched filters for the merger waves vs using a noise monitoring search. Plotted on the horizontal axis is the number $\mathcal{N}_{\text {bins }}=2 T \Delta f$ of independent frequency bins characterizing the space of signals one searches for; $T$ is the maximum expected signal duration and $\Delta f$ is the frequency bandwidth. The vertical axis shows gain factor $\mathcal{R}$ in event rate. This gain factor depends on the number of statistically independent waveform shapes in the set of signals one is searching for, which is currently unknown. This number of waveform shapes can be characterized by the effective dimension $\mathcal{N}_{d}$ of the manifold of signals; cf. Eq. (6.13). The solid line shows the gain factor in the limit in which the number of waveform shapes is small $\left(\mathcal{N}_{d}=0\right)$; it is an upper limit on the gain factor obtainable from matched filtering. The lower two dashed lines show the gain factor when $\mathcal{N}_{d}=5$ and $\mathcal{N}_{d}=10$. Our best estimate of $\mathcal{N}_{\text {bins }}$ is roughly 30 , corresponding to $T=50 M$ and $\Delta f=1 /(\pi M)$; it is unlikely to be much larger than 100 (Sec. III E). This plot can be generated by combining Eqs. (2.27), (2.28), (2.8) and (6.13) of the text, with the parameter values $\mathcal{N}_{\text {start-times }}=10^{8}$ and $\epsilon=10^{-3}$.

over those found from inspiral and ringdown searches for ground-based interferometers. (For LISA, the expected SNRs are so high that the availability of merger templates will likely have no impact on event detection rates.)

\section{CONCLUSIONS}

It seems quite likely that gravitational waves from merging BBH systems will be detected by the ground-based interferometers that are now under construction. Initial LIGO interferometers will be able to detect low mass $\left(\Sigma 30 M_{\odot}\right)$ coalescences of equal-mass BBHs to about $200 \mathrm{Mpc}$ via their inspiral waves, and higher mass $\left(100 M_{\odot} \lesssim M\right.$ $\$ 700 M_{\odot}$ ) systems to about $200 \mathrm{Mpc}$ via their ringdown waves. Advanced LIGO interferometers will be able to detect equal-mass $\mathrm{BBH}$ coalescences in the mass range $10 M_{\odot} \lesssim M \lessgtr 300 M_{\odot}$ to $z \sim 1 / 2$ via their inspiral waves and higher mass $\left(200 M_{\odot} \leq M \leq 3000 M_{\odot}\right)$ systems to $z \sim 1$ via their ringdown waves. For non-equal-mass BBHs, these distances will be reduced by a factor of about $\sqrt{4 \mu / M}$ for inspiral signals and about $4 \mu / M$ for ringdown signals.

Searches for massive BBHs $\left(M \geq 50 M_{\odot}\right.$ for LIGOVIRGO) based on merger waves could increase the range of the interferometers by a additional factor of $\sim 2$, without requiring detailed knowledge of the waveform shapes. It seems likely that BBH coalescences will be detected early in the gradual improvement towards advanced interferometers, and there is a strong possibility that they will be the first sources of gravitational radiation to be detected.

Theoretical template waveforms obtained from numerical relativity supercomputer simulations will be crucial for analyzing the measured merger waves. A match of the detected waveform with a predicted waveform would be a triumph for the theory of general relativity and an absolutely unambiguous signature of the existence of black holes. A complete set of such theoretical templates would also aid the search for BBHs, but not by a large amount.

The space-based interferometer LISA will be an extremely high precision instrument for studying the coalescences of supermassive BBHs. Coalescences with masses in the range $10^{6} M_{\odot} \lesssim(1+z) M \lessgtr 10^{9} M_{\odot}$ should be detectable out to $z \sim 10$ with very large SNRs $\left(\geq 10^{3}\right)$, via their merger and ringdown waves. Additionally, systems in the mass range $10^{4} M_{\odot} \lesssim(1+z) M \lesssim 3 \times 10^{7} M_{\odot}$ should be detected to similar distances and with SNRs $\gtrsim 10^{2}$ via their inspiral waves.

\section{ACKNOWLEDGMENTS}

We thank Kip Thorne for suggesting this project to us, for being a constant source of ideas and encouragement along the way, and for detailed comments on the paper's content and presentation. We also thank Patrick Brady and David Chernoff for some helpful conversations, and Tom Prince and Chip Sumner for locating a well-hidden typographical error in one of our equations. This research was supported by NSF Grants PHY-9220644, PHY-9408378, PHY-9424337, and PHY-9514726, and by NASA Grant NAGW-4268. S.A.H. gratefully acknowledges the support of the National Science Foundation Graduate Program. É. É. Flanagan would like to thank the Enrico Fermi Institute for financial support.

\section{APPENDIX A: ENERGY SPECTRUM FOR RINGDOWN WAVES}

There is a subtlety in calculating the SNR for the ringdown waves, related to the fact that the SNR squared does not accumulate locally in the time domain. In order to explain this subtlety, let us focus not on the angle-averaged SNR squared which was our main concern in the body of the paper, but rather on the SNR squared obtained in one interferometer from a specific source with specific relative angular orientations. In this case the waveform $h(t)$ seen in the interferometer, for $t>0$, is of the form

$$
h(t)=h_{0} \cos \left(2 \pi f_{\mathrm{qnr}} t+\psi_{0}\right) e^{-t / \tau}
$$

for some constants $h_{0}$ and $\psi_{0}$, while $h(t)$ is the (unknown) merger waveform for $t<0$.

Let us also focus first on the simple, idealized case of white noise, $S_{h}(f)=S_{h}=$ const. Then, the SNR squared (2.7) accumulates locally in time: 


$$
\rho^{2}=\frac{2}{S_{h}} \int_{-\infty}^{\infty} d t h(t)^{2}
$$

Hence, for white noise, the SNR squared from the ringdown is clearly unambiguously given by

$$
\begin{aligned}
\rho_{\text {ringdown }}^{2} & =\frac{2}{S_{n}} \int_{0}^{\infty} h_{0}^{2} \cos ^{2}\left(2 \pi f_{\mathrm{qnr}} t+\psi_{0}\right)^{2} e^{-2 t / \tau} \\
& =\frac{h_{0}^{2} \tau}{2 S_{h}}\left[1+\frac{\cos \left(2 \psi_{0}\right)-Q \sin \left(2 \psi_{0}\right)}{1+Q^{2}}\right] \\
& \approx \frac{h_{0}^{2} \tau}{2 S_{h}}[1+O(1 / Q)],
\end{aligned}
$$

where $Q=\pi f_{\mathrm{qnr}} \tau$. Now consider the case when the noise is not exactly white. Naively, we expect that in the Fourier domain the energy spectrum of the ringdown signal will be a resonance curve that peaks at $f=f_{\text {qnr }}$ with width $\sim f_{\text {qnr }} / Q$. Thus, for large $Q$ we would expect that most of the SNR squared will be accumulated near $f=f_{\text {qnr }}$, unless the noise spectrum varies very strongly with frequency. Moreover, if the noise spectrum $S_{h}(f)$ does not vary much over the bandwidth $\sim f_{\text {qnr }} / Q$ of the resonance peak, then we would expect the formula (A3) to be valid to a good approximation, with $S_{h}$ replaced by $S_{h}\left(f_{\text {qnr }}\right)$. We show below that this is indeed the case: under such circumstances, Eq. (A3) is fairly accurate, and the resulting approximate ringdown SNR is embodied in our approximate delta-function energy spectrum (3.19) and in Eqs. (B13)-(B17) of Appendix B [106].

In many cases of interest, it will indeed be true that most of the SNR squared for ringdown waves will be accumulated in the vicinity of the resonance peak, so that the SNR will approximately be given by Eq. (A3). However, this will not always be the case. For instance, suppose that we were lucky enough that two $10^{5} M_{\odot}$ black holes were to merge at the center of our own galaxy. Would such an event be detectable by advanced LIGO interferometers? Clearly, most of the power in the ringdown waves in this case would be far below the LIGO-VIRGO waveband. However, given that the merger is only at $\sim 10 \mathrm{kpc}$, one might hope to be able to detect the tail of the ringdown waves that extends upwards in frequency into the LIGO-VIRGO waveband. Or consider the detectability of a ringdown of a nearby $10^{3} M_{\odot}$ black hole by LISA. In this case most of the ringdown power is concentrated at frequencies above LISA's optimum waveband, and the detectability of the signal is determined by the amount of power in the low frequency tail of the ringdown. In such cases, it is clearly necessary to understand the power carried in the ringdown waves at frequencies far from the resonant frequency.

Normally, such an understanding is obtained simply by taking a Fourier transform of the waveform $h(t)$. In the case of ringdown waves from $\mathrm{BBH}$ mergers, however, the waveform for $t<0$ is the unknown, merger waveform. In order to obtain the SNR squared from the ringdown signal alone, one might guess that the appropriate thing to do is to take $h(t)$ $=0$ for $t<0$, and insert this together with Eq. (A1) into the standard formula (2.7) for the signal to noise squared. However, the resulting energy spectrum has unrealistic high fre- quency behavior due to a discontinuity in $h(t)$ at $t=0$ [or a discontinuity in $h^{\prime}(t)$ at $t=0$ in the case $\psi_{0}=\pi / 2$ ], and the resulting SNRs can in some cases differ from the correct values (see below) by factors $\gtrsim 10$. Other choices for $h(t)$ for $t<0$ [for instance $h(t)=h(0)$ ] get around this problem but instead have unrealistic low frequency behavior. In any case, it is clear that these choices are somewhat ad hoc and that there should be some more fundamental, unique way to calculate the SNR.

We now explain the correct method to calculate the SNR. The question that effectively is being asked is, what is the probability that there is a ringdown waveform present in the data stream, starting at (say) $t=0$ ? This probability is to be calculated given only the data from $t>0$, without using the measured data from $t<0$ which is contaminated by the unknown merger waveform. To do this one must effectively integrate over all possible realizations of the noise for $t<0$. The necessity for such an integration is illustrated by the following simple analogy. Suppose that one is measuring two real variables, $h_{+}$("waveform for positive $t$ ") and $h_{-}$ ("waveform for negative $t$ "), and that the measured values of these variables are $\bar{h}_{+}$and $\bar{h}_{-}$. Suppose that because of the noise in the measurement process, the probability distribution for the true values of these parameters given their measured values is

$$
\begin{aligned}
p\left(h_{+}, h_{-}\right)= & \frac{1}{2 \pi \sigma^{2}} \exp \left\{-\frac{1}{2 \sigma^{2}}\left[\left(h_{+}-\bar{h}_{+}\right)^{2}+\left(h_{-}-\bar{h}_{-}\right)^{2}\right.\right. \\
& \left.\left.+2 \varepsilon\left(h_{+}-\bar{h}_{+}\right)\left(h_{-}-\bar{h}_{-}\right)\right]\right\} .
\end{aligned}
$$

Thus, $h_{+}$and $h_{-}$are Gaussian distributed about their means $\bar{h}_{+}$and $\bar{h}_{-}$, and they are correlated. If we assume that $h_{-}$ $=0$ [analogous to assuming $h(t)=0$ for $t<0$ ], we obtain, for the probability distribution for $h_{+}$,

$$
p\left(h_{+} \mid h_{-}=0\right)=\frac{1}{\sqrt{2 \pi} \sigma} e^{-\left(h_{+}-\bar{h}_{+}^{\prime}\right)^{2} /(2 \sigma)^{2}},
$$

where $\bar{h}_{+}^{\prime}=\bar{h}_{+}-\varepsilon \bar{h}_{-}$. By contrast, if we instead calculate the probability distribution for $h_{+}$alone by integrating over $h_{-}$we find

$$
p\left(h_{+}\right)=\frac{1}{\sqrt{2 \pi} \sigma^{*}} e^{-\left(h_{+}-\bar{h}_{+}\right)^{2} /\left(2 \sigma^{*}\right)^{2}},
$$

where $\sigma^{*}=\sigma / \sqrt{1-\varepsilon^{2}}$. It is clear in this simple example that one should use the reduced distribution (A6) rather than the distribution (A5). Note also that the widths of the probability distributions (A5) and (A6) are different, and that the correct distribution (A6) could not have been obtained from the joint distribution (A4) for any assumed choice of $h_{-}$. 
Turn now to the analogous situation for random processes. If $n(t)$ is the interferometer noise, let $C_{n}(\Delta t)$ $\equiv\langle n(t) n(t+\Delta t)\rangle$ denote the autocorrelation function. Define the inner product

$$
\left\langle h_{1} \mid h_{2}\right\rangle \equiv \int_{0}^{\infty} d t \int_{0}^{\infty} d t^{\prime} K\left(t, t^{\prime}\right) h_{1}(t) h_{2}\left(t^{\prime}\right)
$$

on the space of functions $h(t)$ for $t>0$, where the kernel $K\left(t, t^{\prime}\right)$ is determined from

$$
\int_{0}^{\infty} d t^{\prime \prime} K\left(t, t^{\prime \prime}\right) C_{n}\left(t^{\prime \prime}-t^{\prime}\right)=\delta\left(t-t^{\prime}\right)
$$

for $t, t^{\prime} \geqslant 0$. The quantity $K\left(t, t^{\prime}\right)$ is analogous to the modified width $\sigma^{*}$ in Eq. (A6) above. Using the inner product (A7), the usual theory of matched filtering $[59,60]$ can be applied to random processes on the half line $t>0$. Thus, if $s(t)$ is the interferometer output and $h(t)$ is the waveform (A1), the detection statistic is $Y=\langle s \mid h\rangle$, and the SNR squared for the measurement is

$$
\begin{aligned}
\rho^{2} & \equiv \frac{E[Y]^{2}}{E\left[Y^{2}\right]-E[Y]^{2}} \\
& =\langle h \mid h\rangle \\
& =\int_{0}^{\infty} d t \int_{0}^{\infty} d t^{\prime} K\left(t, t^{\prime}\right) h(t) h\left(t^{\prime}\right),
\end{aligned}
$$

where $E[\cdots]$ means expectation value. If we define

$$
G\left(f, f^{\prime}\right)=\int_{0}^{\infty} d t \int_{0}^{\infty} d t^{\prime} e^{2 \pi i f t} e^{-2 \pi i f^{\prime} t^{\prime}} K\left(t, t^{\prime}\right)
$$

and

$$
\widetilde{h}(f)=\int_{0}^{\infty} e^{2 \pi i f t} h(t) d t
$$

the SNR squared can be rewritten as

$$
\rho^{2}=\int_{-\infty}^{\infty} d f \int_{-\infty}^{\infty} d f^{\prime} \tilde{h}(f)^{*} G\left(f, f^{\prime}\right) \widetilde{h}\left(f^{\prime}\right) .
$$

Note that the Fourier transform $G\left(f, f^{\prime}\right)$ of $K\left(t, t^{\prime}\right)$ is not proportional to $\delta\left(f-f^{\prime}\right) / S_{h}(f)$ but instead is in general non-diagonal in frequency.

The formula (A12) resolves the ambiguities discussed above in the method of calculating the ringdown SNR; the result does not require a choice of the waveform $h(t)$ for $t$ $<0$. Unfortunately, the final answer (A12) is complicated in the sense that it cannot be expressed in the form (2.30) for any effective energy spectrum $d E / d f$. This is somewhat inconvenient for the purposes of this paper: the wave's energy spectrum is a useful and key tool for visualizing and understanding the SNRs. Clearly, an approximate, effective energy spectrum (to the extent that one exists) would be very useful. We now turn to a derivation of such an approximate, effective energy spectrum, namely the spectrum (3.18) which is used throughout the body of this paper.
We start our derivation by describing an alternative method of calculating the exact ringdown SNR given by Eqs. (A1) and (A9). It is straightforward to show that the quantity (A9) can be obtained by (i) choosing any waveform $h(t)$ for $t<0$, (ii) calculating the SNR from the usual formula (2.7), and (iii) minimizing over all choices of the function $h(t)$ on the negative real axis. We have experimented with several choices of $h(-t)$ for $t>0$, namely $h(-t)=0, h(-t)$ $=h(0), h(-t)=h(t)$. We found that the SNR obtained by minimizing over these choices is always (for the entire black hole mass ranges discussed in Sec. V) within a few tens of a percent of the SNR obtained from the following prescription: (i) Assume that $h(t)$ for negative $t$ is identical to the waveform for positive $t$ except for the sign of $t / \tau$; i.e., that

$$
h(t)=h_{0} \cos \left(2 \pi f_{\mathrm{qnr}} t+\psi_{0}\right) e^{-|t| / \tau}
$$

for positive and negative $t$. (ii) Calculate the total SNR using the standard formula (2.7). (iii) Divide by a correction factor of $\sqrt{2}$ in amplitude to compensate for the doubling up. This prescription gives the correct, exact result (A9) for white noise. For more realistic noise curves, the errors of a few tens of a percent resulting from this prescription are unimportant compared to the uncertainty in the overall amplitude $\mathcal{A}$ of the ringdown signal. Moreover, the resulting SNR values multiplied by $\sqrt{2}$ are an upper bound for the true SNR [since if our ad hoc choice of $h(t)$ for $t<0$ happened to be exactly right, then the prescription would underestimate the SNR by $\sqrt{2}$ ].

We now explain how to obtain the energy spectrum (3.18) from the above approximate prescription. From Eqs. (2.31) and (3.15) it can be seen that the waveform as seen in one interferometer, before angle averaging, is given by Eq. (A1) with

$$
h_{0} e^{i \psi_{0}}=\frac{\mathcal{A} M}{r}\left[F_{+}(\theta, \varphi, \psi)+i F_{\times}(\theta, \varphi, \psi)\right]_{2} S_{2}^{2}(\iota, \beta, a) e^{i \varphi_{0}} .
$$

Here the angles $\theta, \varphi, \psi, \iota$ and $\beta$ have the meanings explained in Sec. II C. Let us now insert the waveform (A1) into the formula (A9) for the exact SNR, and then average over the angles $\theta, \varphi, \psi, \iota$ and $\beta$ using Eqs. (2.34) and (3.16). This yields for the angle-averaged, exact SNR squared

$$
\left\langle\rho_{\text {exact }}[h(t)]^{2}\right\rangle=\frac{1}{20 \pi}\left\{\rho_{\text {exact }}\left[h_{+, 0}(t)\right]^{2}+\rho_{\text {exact }}\left[h_{\times, 0}(t)\right]^{2}\right\},
$$

where $\rho_{\text {exact }}[h(t)]$ denotes the exact SNR functional (A9) and

$$
\begin{aligned}
h_{+, 0}(t) & =\frac{\mathcal{A} M}{r} \cos \left(2 \pi f_{\mathrm{qnr}} t\right) e^{-t / \tau} \\
h_{\times, 0}(t) & =\frac{\mathcal{A} M}{r} \sin \left(2 \pi f_{\mathrm{qnr}} t\right) e^{-t / \tau},
\end{aligned}
$$

for $l>0$. Now, for each of the two terms on the right-hand side of Eq. (A15), we make the approximation discussed above consisting of using Eqs. (2.7) and (A13) and dividing by 2 . This yields 


$$
\left\langle\rho_{\text {exact }}[h(t)]^{2}\right\rangle \approx \frac{1}{10 \pi} \int_{0}^{\infty} d f \frac{\left[\left|\widetilde{h}_{+, 0}(f)\right|^{2}+\left|\widetilde{h}_{+, 0}(f)\right|^{2}\right]}{S_{h}(f)},
$$

where it is understood that $h_{+, 0}$ and $h_{\times, 0}$ have been extended to negative $t$ in the manner of Eq. (A13). Finally, evaluating the Fourier transforms yields an angle averaged SNR squared of the form (2.30), with the energy spectrum given by Eq. (3.18).

\section{APPENDIX B: SIGNAL-TO-NOISE RATIO FORMULAS}

In this appendix, we give the details of our SNR calculations. Note that throughout this appendix we use "M $M_{\odot}$ ", (mega solar-mass) as shorthand for $10^{6} M_{\odot}$.

\section{Inspiral}

To calculate the angle-averaged SNR squared for the inspiral, we insert the inspiral energy spectrum (3.14) and our parameterized model (4.1) of an interferometer's noise spectrum into Eq. (2.30), and integrate from $f=f_{s}$ to $f=f_{\text {merge }}$. The result is

$$
\left\langle\rho^{2}\right\rangle= \begin{cases}\mathcal{F}_{i}(M, z, D)\left[9 \alpha^{1 / 3}-\frac{36}{5} \alpha^{-1 / 3}-\frac{4}{5} \alpha^{-1 / 3} v^{10 / 3}-\alpha^{3}\left(\frac{f_{s}}{f_{m}}\right)^{8 / 3}\right], & \alpha f_{m} \leqslant f_{\text {merge }} /(1+z), \\ \mathcal{F}_{i}(M, z, D)\left[9 \alpha^{1 / 3}-8\left(\frac{v}{\alpha}\right)^{1 / 3}-\alpha^{3}\left(\frac{f_{s}}{f_{m}}\right)^{8 / 3}\right], & f_{m} / \alpha \leqslant f_{\text {merge }} /(1+z)<\alpha f_{m}, \\ \mathcal{F}_{i}(M, z, D)\left[\alpha^{1 / 3}\left(\frac{\alpha^{2}}{v}\right)^{8 / 3}-\alpha^{3}\left(\frac{f_{s}}{f_{m}}\right)^{8 / 3}\right], & f_{s} \leqslant f_{\text {merge }} /(1+z)<f_{m} / \alpha, \\ 0, & f_{\text {merge }} /(1+z)<f_{s},\end{cases}
$$

where

$$
v \equiv \frac{(1+z) \alpha f_{m}}{f_{\text {merge }}}
$$

and

$$
\mathcal{F}_{i}(M, z, D)=\frac{[(1+z) M]^{5 / 3}[4 \mu / M]}{80 \pi^{4 / 3} D(z)^{2} h_{m}^{2} f_{m}^{1 / 3}} .
$$

Here $D(z)$ is the luminosity distance to the source, $f_{s}, \alpha, f_{m}$ and $h_{m}$ are parameters characterizing the detector noise spectrum (4.1), and $f_{\text {merge }}$ is given by Eq. (3.3).

Inserting the values of the noise spectrum parameters from Eq. (4.2) for initial LIGO interferometers, we obtain the following numerical values for the SNR in the equal-mass case $\mu=M / 4$ :

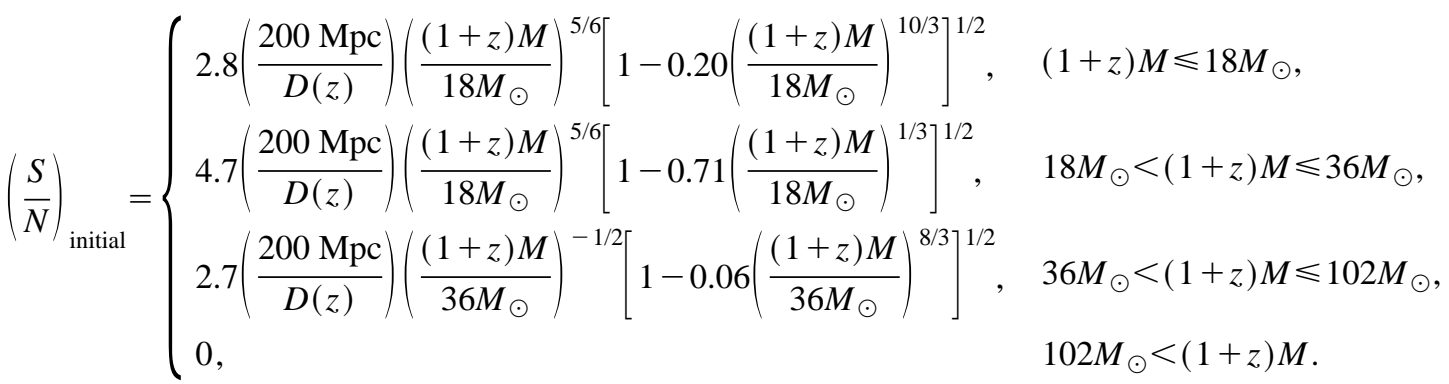

For the noise curve parameters (4.3) appropriate for advanced LIGO interferometers we obtain

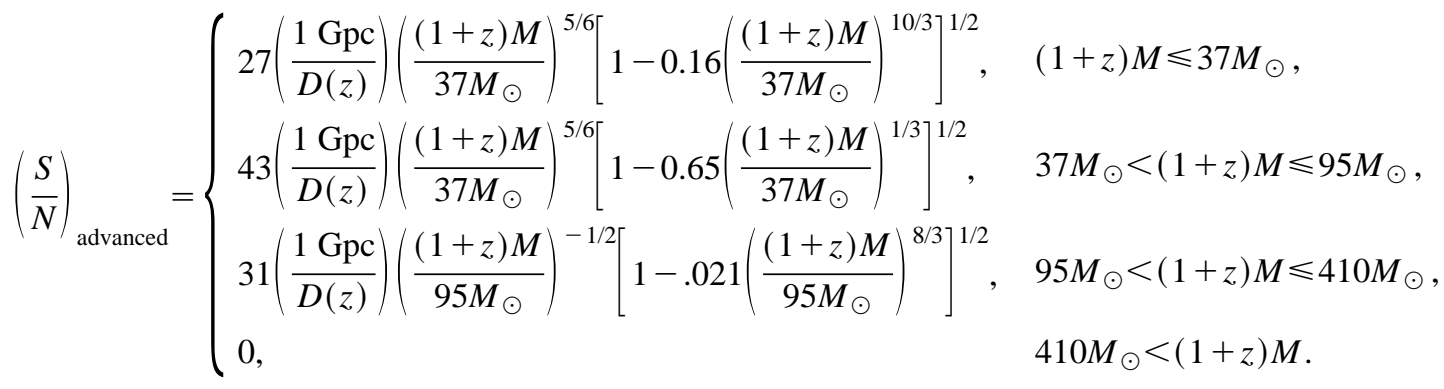


As explained in Sec. V A, the calculation of the inspiral SNR for LISA differs from the other SNR calculations in the following way. If one were to integrate over the whole frequency domain in the interferometer waveband up to $f=f_{\text {merge }}$ (as is done for the initial and advanced interferometers in LIGO), in some cases one would obtain the SNR for a measurement of several hundred years duration, which is obviously irrelevant. Thus, it is necessary to restrict the integral over frequency in Eq. (2.30) to the domain that corresponds to, say, $1 \mathrm{yr}$ of observation when calculating inspiral LISA SNRs. Using the Newtonian relationship for the rate of frequency sweep, we obtain, for the frequency at time $T$ before merger in the equal-mass case,

$$
f_{\text {insp }}(T)=\left[f_{\text {merge }}^{-8 / 3}+\frac{64}{5} \pi^{8 / 3} M^{5 / 3}(1+z)^{5 / 3} T\right]^{-3 / 8} .
$$

Binaries of redshifted total mass $(1+z) M$ larger than about $5 \times 10^{5} M_{\odot}$ enter the LISA waveband at $f=f_{s}=10^{-4} \mathrm{~Hz}$ less than $1 \mathrm{yr}$ before merger, while binaries of smaller redshifted mass spend more than $1 \mathrm{yr}$ in the LISA waveband. To calculate the SNR, we insert Eq. (3.14) into Eq. (2.30) and integrate numerically from the larger of $f_{s}$ and $f_{\text {insp }}(1 \mathrm{yr})$ to $f_{\text {merge }}$. The resulting SNR values are shown in Fig. 6. We also show in Fig. 6 the SNR obtained from 1 yr of observation 100 yr before the final merger, obtained by integrating from $f_{\text {insp }}(100 \mathrm{yr})$ to $f_{\text {insp }}(99 \mathrm{yr})$, as well as a similar curve for $1000 \mathrm{yr}$ prior to merger.

Equation (B1) applies to LISA only for $(1+z) M \geqslant 5 \times 10^{5} M_{\odot}$. By combining Eqs. (B1) and (4.4) for $(1+z) M \gtrsim$ $5 \times 10^{5} M_{\odot}$ together with an approximate fit to Fig. 6 for $(1+z) M \lesssim 10^{5} M_{\odot}$ we obtain, for the SNR from the last year of inspiral in the equal-mass case,

$$
\left(\frac{S}{N}\right)_{\text {LISA }} \approx \begin{cases}1.5 \times 10^{4}\left(\frac{1 \mathrm{Gpc}}{D(z)}\right)\left(\frac{(1+z) M}{0.5 \mathrm{M} M_{\odot}}\right), & 100 M_{\odot} \lesssim(1+z) M \lessgtr 0.5 \mathrm{M} M_{\odot}, \\ 1.9 \times 10^{4}\left(\frac{1 \mathrm{Gpc}}{D(z)}\right)\left(\frac{(1+z) M}{0.5 \mathrm{M} M_{\odot}}\right)^{5 / 6}\left[1-0.38\left(\frac{(1+z) M}{0.5 \mathrm{M} M_{\odot}}\right)^{1 / 3}\right]^{1 / 2}, & 0.5 \mathrm{M} M_{\odot}<(1+z) M \leqslant 6.0 \mathrm{M} M_{\odot}, \\ 5.0 \times 10^{4}\left(\frac{1 \mathrm{Gpc}}{D(z)}\right)\left(\frac{(1+z) M}{6 \mathrm{M} M_{\odot}}\right)^{-1 / 2}\left[1-0.006\left(\frac{(1+z) M}{6 \mathrm{M} M_{\odot}}\right)^{8 / 3}\right]^{1 / 2}, & 6.0 \mathrm{M} M_{\odot}<(1+z) M \leqslant 41 \mathrm{M} M_{\odot}, \\ 0, & 41 \mathrm{M} M_{\odot}<(1+z) M .\end{cases}
$$

\section{Merger}

To calculate the merger SNR we use the energy spectrum (3.13) and follow the same procedure as above. The result is

$$
\left\langle\rho^{2}\right\rangle= \begin{cases}\mathcal{F}_{m}\left(\epsilon_{m}, M, z, D\right) v^{3}\left[\frac{\kappa^{3}-1}{\kappa^{3}}\right] & f_{\text {merge }} /(1+z) \geq \alpha f_{m} \\ \mathcal{F}_{m}\left(\epsilon_{m}, M, z, D\right)\left[3 \ln v-\frac{v^{3}-\kappa^{3}}{\kappa^{3}}\right] & f_{m} / \alpha \leq f_{\text {merge }} /(1+z)<\alpha f_{m} \leq f_{\mathrm{qnr}} /(1+z) \\ \mathrm{I}, \mathrm{A} \mathcal{F}_{m}\left(\epsilon_{m}, M, z, D\right)\left[2-\frac{\alpha^{6}}{v^{3}}-\frac{v^{3}}{\kappa^{3}}+6 \ln \alpha\right] & f_{\text {merge }} /(1+z) \leq f_{m} / \alpha<\alpha f_{m} \leq f_{\mathrm{qnr}} /(1+z) \\ \mathrm{L} \mathcal{F}_{m}\left(\epsilon_{m}, M, z, D\right)[3 \ln \kappa] & f_{m} / \alpha \leq f_{\text {merge }} /(1+z)<f_{\mathrm{qnr}} /(1+z) \leq \alpha f_{m} \\ \mathrm{I} \mathcal{F}_{m}\left(\epsilon_{m}, M, z, D\right)\left[2-\left(\frac{\alpha f_{s}}{f_{m}}\right)^{3}-\frac{v^{3}}{\kappa^{3}}+6 \ln \alpha\right] & f_{\text {merge }} /(1+z) \leq f_{s}<f_{m} / \alpha<\alpha f_{m} \leq f_{\mathrm{qnr}} /(1+z) \\ \mathrm{A}, \mathrm{L} \mathcal{F}_{m}\left(\epsilon_{m}, M, z, D\right)\left[1+3 \ln \left(\frac{\kappa \alpha^{2}}{v}\right)-\frac{\alpha^{6}}{v^{3}}\right] & f_{s} \leq f_{\text {merge }} /(1+z)<f_{m} / \alpha \leq f_{\mathrm{qnr}} /(1+z) \\ \mathrm{I}, \mathrm{A} \mathcal{F}_{m}\left(\epsilon_{m}, M, z, D\right)\left[1-\left(\frac{\alpha f_{s}}{f_{m}}\right)^{3}+3 \ln \left(\frac{\kappa \alpha^{2}}{v}\right)\right] & f_{\text {merge }} /(1+z) \leq f_{s}<f_{m} / \alpha \leq f_{\mathrm{qnr}} /(1+z) \\ \mathbf{L} \mathcal{F}_{m}\left(\epsilon_{m}, M, z, D\right)\left[\alpha^{6} v^{-3}\left(\kappa^{3}-1\right)\right] & f_{s} \leq f_{\text {merge }} /(1+z)<f_{\mathrm{qnr}} /(1+z) \\ \mathcal{F}_{m}\left(\epsilon_{m}, M, z, D\right)\left[\left(\frac{\kappa \alpha^{2}}{v}\right)^{3}-\left(\frac{\alpha f_{s}}{f_{m}}\right)^{3}\right] & f_{\text {merge }} /(1+z) \leq f_{s}<f_{\mathrm{qnr}} /(1+z) \leq f_{m} / \alpha \\ 0 & f_{\mathrm{qnr}} /(1+z)<f_{s} .\end{cases}
$$

Here $v$ is given by Eq. (B2), $\boldsymbol{\epsilon}_{m}$ is the fraction of total mass energy radiated during the merger (which we have also denoted by $\epsilon_{\text {merger }}$ in the body of the paper), $\kappa \equiv f_{\text {qnr }} / f_{\text {merge }}$, and

$$
\mathcal{F}_{m}\left(\epsilon_{m}, M, z, D\right)=\frac{2 \epsilon_{m} M(1+z)^{2}[4 \mu / M]^{2}}{15 \pi^{2} D(z)^{2} h_{m}^{2} f_{\text {merge }}(\kappa-1)} .
$$

Lines marked with the superscript "I" turn out to hold for the initial LIGO interferometer parameters, those with "A" hold for advanced LIGO interferometer parameters, and those with ' $\mathrm{L}$ ' hold for LISA. 
Using the numerical values of the noise curve parameters (4.2) for initial LIGO interferometers, and Eqs. (3.3), (3.4), (B2), and (B8) we find, for the initial LIGO interferometers in the equal-mass case,

$$
\left(\frac{S}{N}\right)_{\text {initial }}= \begin{cases}1.5\left(\frac{\epsilon_{m}}{0.1}\right)^{1 / 2}\left(\frac{200 \mathrm{Mpc}}{D(z)}\right)\left(\frac{(1+z) M}{18 M_{\odot}}\right)^{5 / 2} & (1+z) M \leq 18 M_{\odot} \\ 1.5\left(\frac{\epsilon_{m}}{0.1}\right)^{1 / 2}\left(\frac{200 \mathrm{Mpc}}{D(z)}\right)\left(\frac{(1+z) M}{18 M_{\odot}}\right) & \\ \quad \times\left[1+3 \ln \left(\frac{(1+z) M}{18 M_{\odot}}\right)-3.6 \times 10^{-3}\left(\frac{(1+z) M}{18 M_{\odot}}\right)^{3}\right]^{1 / 2} & 18 M_{\odot}<(1+z) M \leq 36 M_{\odot} \\ 6.1\left(\frac{\epsilon_{m}}{0.1}\right)^{1 / 2}\left(\frac{200 \mathrm{Mpc}}{D(z)}\right)\left(\frac{(1+z) M}{36 M_{\odot}}\right) & \\ \quad \times\left[1+.23\left(\frac{(1+z) M}{36 M}\right)^{-3}-0.007\left(\frac{(1+z) M}{36 M}\right)^{3}\right]^{1 / 2} & 36 M_{\odot}<(1+z) M \leq 102 M_{\odot} \\ 17.3\left(\frac{\epsilon_{m}}{0.1}\right)^{1 / 2}\left(\frac{200 M p c}{D(z)}\right)\left(\frac{(1+z) M}{102 M_{\odot}}\right) & \\ \quad \times\left[1-.17\left(\frac{(1+z) M}{102 M_{\odot}}\right)^{3}\right]^{1 / 2} & 102 M_{\odot}<(1+z) M \leq 118 M_{\odot} \\ 9.9\left(\frac{\epsilon_{m}}{0.1}\right)^{1 / 2}\left(\frac{200 \mathrm{Mpc}}{D(z)}\right)\left(\frac{(1+z) M}{118 M_{\odot}}\right)\left[1-3.1 \ln \left(\frac{(1+z) M}{230 M}\right)\right]^{1 / 2} & 118 M_{\odot}<(1+z) M \leq 230 M_{\odot} \\ 20\left(\frac{\epsilon_{m}}{0.1}\right)^{1 / 2}\left(\frac{200 \mathrm{Mpc}}{D(z)}\right)\left(\frac{(1+z) M}{230 M_{\odot}}\right)^{-1 / 2}\left[1-0.04\left(\frac{(1+z) M}{230 M}\right)^{3}\right]^{1 / 2} & 230 M_{\odot}<(1+z) M \leq 660 M_{\odot} \\ 0 \quad & 660 M_{\odot}<(1+z) M .\end{cases}
$$

Similarly using Eq. (4.3) we find, for advanced LIGO interferometers,

$$
\left(\frac{S}{N}\right)_{\text {advanced }}= \begin{cases}13\left(\frac{\epsilon_{m}}{0.1}\right)^{1 / 2}\left(\frac{1 \mathrm{Gpc}}{D(z)}\right)\left(\frac{(1+z) M}{37 M_{\odot}}\right)^{5 / 2} & (1+z) M \leq 37 M_{\odot} \\ 13\left(\frac{\epsilon_{m}}{0.1}\right)^{1 / 2}\left(\frac{1 \mathrm{Gpc}}{D(z)}\right)\left(\frac{(1+z) M}{37 M_{\odot}}\right) & \\ \quad \times\left[1+3 \ln \left(\frac{(1+z) M}{37 M_{\odot}}\right)-3.6 \times 10^{-3}\left(\frac{(1+z) M}{37 M_{\odot}}\right)^{3}\right]^{1 / 2} & 37 M_{\odot}<(1+z) M \leq 95 M_{\odot} \\ 76\left(\frac{\epsilon_{m}}{0.1}\right)^{1 / 2}\left(\frac{1 \mathrm{Gpc}}{D(z)}\right)\left(\frac{(1+z) M}{95 M_{\odot}}\right) & \\ \quad \times\left[1-0.21\left(\frac{(1+z) M}{95 M_{\odot}}\right)^{-3}-0.013\left(\frac{(1+z) M}{95 M_{\odot}}\right)^{3}\right]^{1 / 2} & 95 M_{\odot}<(1+z) M \leq 240 M_{\odot} \\ 88\left(\frac{\epsilon_{m}}{0.1}\right)^{1 / 2}\left(\frac{1 \mathrm{Gpc}}{D(z)}\right)\left(\frac{(1+z) M}{240 M_{\odot}}\right) & \\ \quad \times\left[1-3 \ln \left(\frac{(1+z) M}{620 M}\right)-0.061\left(\frac{(1+z) M}{240 M_{\odot}}\right)^{-3}\right]^{1 / 2} & 240 M_{\odot}<(1+z) M \leq 410 M_{\odot} \\ 150\left(\frac{\epsilon_{m}}{0.1}\right)^{1 / 2}\left(\frac{1 \mathrm{Gpc}}{D(z)}\right)\left(\frac{(1+z) M}{410 M_{\odot}}\right)\left[1-3.0 \ln \left(\frac{(1+z) M}{620 M_{\odot}}\right)\right]^{1 / 2} & 410 M_{\odot}<(1+z) M \leq 620 M_{\odot} \\ 220\left(\frac{\epsilon_{m}}{0.1}\right)^{1 / 2}\left(\frac{1 \mathrm{Gpc}}{D(z)}\right)\left(\frac{(1+z) M}{620 M_{\odot}}\right)^{-1 / 2}\left[1-0.013\left(\frac{(1+z) M}{620 M_{\odot}}\right)^{3}\right]^{1 / 2} & 620 M_{\odot}<(1+z) M \leq 2600 M_{\odot} \\ 0 \quad & 2600 M_{\odot}<(1+z) M .\end{cases}
$$

Finally, using the parameters (4.4) appropriate for LISA, we obtain 


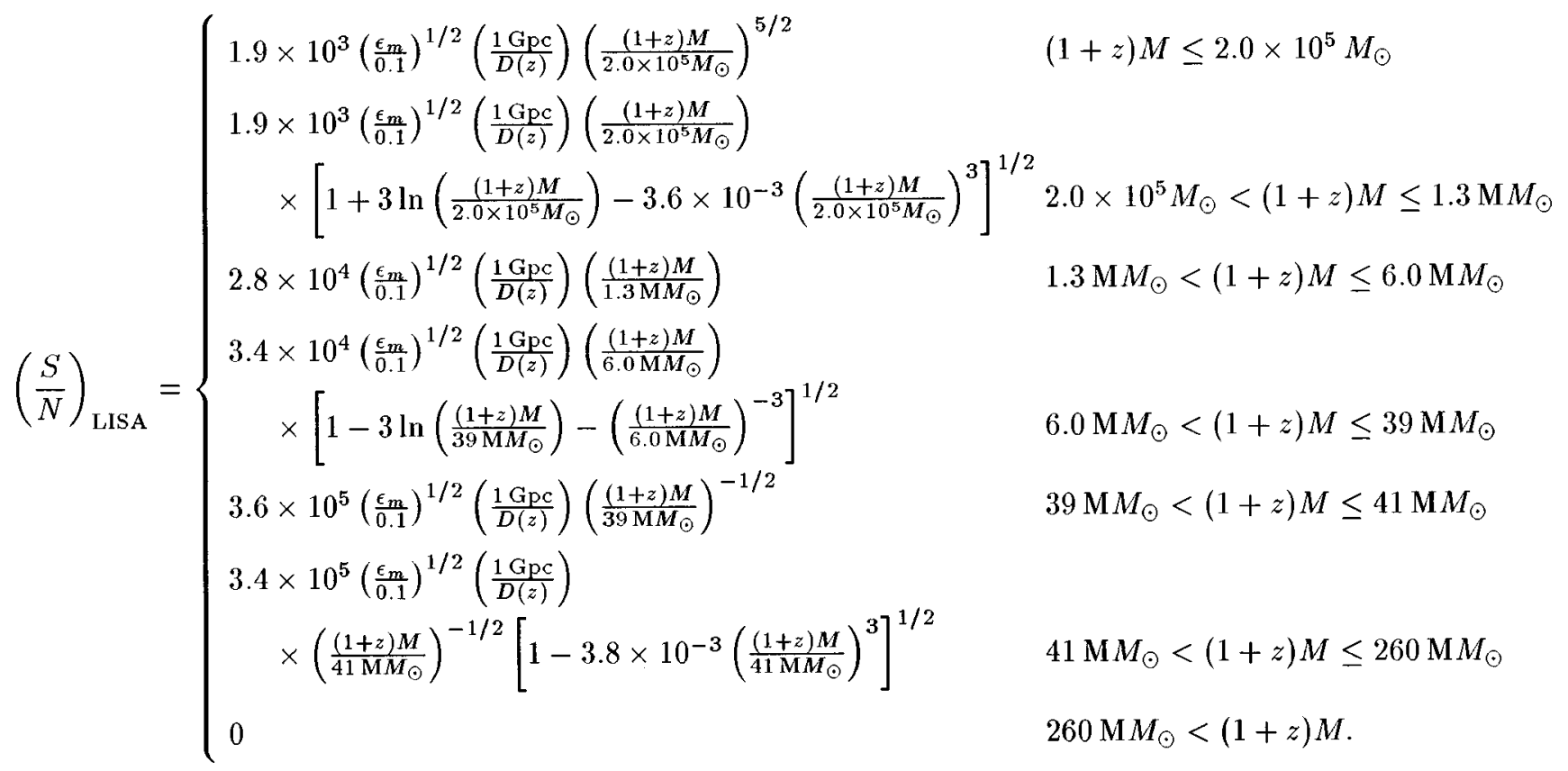

\section{Ringdown}

The ringdown SNRs are calculated a little differently from the inspiral and merger SNRs. First, we use the effective energy spectrum (3.18) which yields an estimate of the true SNR obtainable from the model waveform (3.15) that is accurate to within a few tens of a percent (see Appendix A). Second, the integral over frequency in the SNR formula (2.30) with the noise spectrum (4.1) and the energy spectrum (3.18) cannot easily be evaluated analytically. Hence, we calculated this integral numerically to produce the plots of ringdown SNR versus BBH mass shown in Figs. 4, 5, and 6.

In the remainder of this appendix we derive approximate formulae for the ringdown SNR as a function of mass, by approximating the ringdown energy spectrum as a delta function at the ringdown frequency [cf. Eq. (3.19)]. This approximation yields (see Appendix A and Ref. [106])

$$
\left\langle\rho^{2}\right\rangle=\frac{(1+z)^{3} M^{2} \mathcal{A}^{2} Q[4 \mu / M]^{2}}{20 \pi^{2} D(z)^{2} f_{\mathrm{qnr}} S_{h}\left[f_{\mathrm{qnr}} /(1+z)\right]} .
$$

Using Eq. (3.17) and the relation (3.22) between the dimensionless coefficient $\mathcal{A}$ and the radiated energy we can rewrite formula (B13) as

$$
\left\langle\rho^{2}\right\rangle=\frac{8}{5} \frac{1}{F(a)^{2}} \epsilon_{r} \frac{(1+z) M}{S_{h}\left[f_{\mathrm{qnr}} /(1+z)\right]}\left[\frac{(1+z) M}{D(z)}\right]^{2}\left[\frac{4 \mu}{M}\right]^{2},
$$

where $\epsilon_{r}=\epsilon_{\text {ringdown }}$ is the fraction of the total mass energy radiated in the ringdown, and

$$
F(a)=1-\frac{63}{100}(1-a)^{3 / 10}
$$

An equivalent formula was previously obtained by Finn [16,107].

We find the following numerical result when we insert our assumed values $\epsilon_{r}=0.03$ and $a=0.98$ for the ringdown signal together with the parameters for the initial LIGO interferometer noise curve in the equal-mass case:

$$
\left(\frac{S}{N}\right)_{\text {initial }}= \begin{cases}0.08\left(\frac{\epsilon_{r}}{0.03}\right)^{1 / 2}\left(\frac{200 \mathrm{Mpc}}{D(z)}\right)\left(\frac{(1+z) M}{18 M_{\odot}}\right)^{5 / 2}, & (1+z) M \leqslant 118 M_{\odot}, \\ 8.8\left(\frac{\epsilon_{r}}{0.03}\right)^{1 / 2}\left(\frac{200 \mathrm{Mpc}}{D(z)}\right)\left(\frac{(1+z) M}{118 M_{\odot}}\right), & 118 M_{\odot}<(1+z) M \leqslant 230 M_{\odot}, \\ 17\left(\frac{\epsilon_{r}}{0.03}\right)^{1 / 2}\left(\frac{200 \mathrm{Mpc}}{D(z)}\right)\left(\frac{(1+z) M}{230 M_{\odot}}\right)^{-1 / 2}, & 230 M_{\odot}<(1+z) M \leqslant 660 M_{\odot}, \\ 0, & 660 M_{\odot}<(1+z) M .\end{cases}
$$


The corresponding formulas for advanced LIGO interferometers are

$$
\left(\frac{S}{N}\right)_{\text {advanced }}= \begin{cases}0.71\left(\frac{\epsilon_{r}}{0.03}\right)^{1 / 2}\left(\frac{1 \mathrm{Gpc}}{D(z)}\right)\left(\frac{(1+z) M}{37 M_{\odot}}\right)^{5 / 2}, & (1+z) M \leqslant 240 M_{\odot}, \\ 77\left(\frac{\epsilon_{r}}{0.03}\right)^{1 / 2}\left(\frac{1 \mathrm{Gpc}}{D(z)}\right)\left(\frac{(1+z) M}{240 M_{\odot}}\right), & 240 M_{\odot}<(1+z) M \leqslant 620 M_{\odot}, \\ 200\left(\frac{\epsilon_{r}}{0.03}\right)^{1 / 2}\left(\frac{1 \mathrm{Gpc}}{D(z)}\right)\left(\frac{(1+z) M}{620 M_{\odot}}\right)^{-1 / 2}, & 620 M_{\odot}<(1+z) M \leqslant 2600 M_{\odot}, \\ 0, & 2600 M_{\odot}<(1+z) M .\end{cases}
$$

Finally, the corresponding formulas for LISA are

$$
\left(\frac{S}{N}\right)_{\text {LISA }}= \begin{cases}96\left(\frac{\epsilon_{r}}{0.03}\right)^{1 / 2}\left(\frac{1 \mathrm{Gpc}}{D(z)}\right)\left(\frac{(1+z) M}{0.2 \mathrm{M} M_{\odot}}\right)^{5 / 2}, & (1+z) M \leqslant 1.3 \mathrm{M} M_{\odot}, \\ 1.0 \times 10^{4}\left(\frac{\epsilon_{r}}{0.03}\right)^{1 / 2}\left(\frac{1 \mathrm{Gpc}}{D(z)}\right)\left(\frac{(1+z) M}{1.3 \mathrm{M} M_{\odot}}\right), & 1.3 \mathrm{M} M_{\odot}<(1+z) M \leqslant 39 \mathrm{M} M_{\odot}, \\ 3.1 \times 10^{5}\left(\frac{\epsilon_{r}}{0.03}\right)^{1 / 2}\left(\frac{1 \mathrm{Gpc}}{D(z)}\right)\left(\frac{(1+z) M}{39 \mathrm{M} M_{\odot}}\right)^{-1 / 2}, & 39 \mathrm{M} M_{\odot}<(1+z) M \leqslant 260 \mathrm{M} M_{\odot}, \\ 0, & 260 \mathrm{M} M_{\odot}<(1+z) M .\end{cases}
$$

By comparing Eqs. (B16)-(B18) with Figs. 4-6 it can be seen that the delta-function energy spectrum approximation is fairly good except for $M \geq 3000 M_{\odot}$ for advanced LIGO interferometers and $M \gtrsim 3 \times 10^{8} M_{\odot}$ for LISA. The approximation fails to capture the high mass tails of the SNR curves.

[1] K. S. Thorne, in 300 Years of Gravitation, edited by S. W. Hawking and W. Israel (Cambridge University Press, Cambridge, England, 1987), pp. 330-458.

[2] B. F. Schutz, in "NASA, Relativistic Gravitational Experiments in Space,' 1989, pp. 7-13.

[3] A. Abramovici, W. E. Althouse, R. W. P. Drever, Y. Gürsel, S. Kawamura, F. J. Raab, D. Shoemaker, L. Sievers, R. E. Spero, K. S. Thorne, R. E. Vogt, R. Weiss, S. E. Whitcomb, and M. E. Zucker, Science 256, 325 (1992).

[4] C. Bradaschia et al., Nucl. Instrum. Methods Phys. Res. A 289, 518 (1990); also in Gravitation: A Banff Summer Institute, edited by R. Mann and P. Wesson (World Scientific, Singapore, 1991).

[5] P. Bender et al., "LISA, Laser interferometer space antenna for gravitational wave measurements: ESA Assessment Study Report," 1994.

[6] J. Hough et al., in Gravitational Wave Experiments, proceedings of the Edoardo Amaldi Conference, edited by E. Coccia, G. Pizzella, and F. Ronga (World Scientific, Singapore, 1995), p. 50

[7] P. Bender, I. Ciufolini, K. Danzmann, W. Folkner, J. Hough, D. Robertson, A. Rüdiger, M. Sandford, R. Schilling, B. Schutz, R. Stebbins, T. Summer, P. Touboul, S. Vitale, H. Ward, and W. Winkler, "LISA: Laser Interferometer Space Antenna for the detection and observation of gravitational waves," pre-phase A report, 1995 (unpublished).

[8] L. E. Kidder, C. M. Will, and A. G. Wiseman, Phys. Rev. D 47, 3281 (1993); Class. Quantum Grav. 9, L125 (1992).

[9] G. B. Cook, Phys. Rev. D 50, 5025 (1994). See especially Fig. 3.
[10] K. S. Thorne, in Proceedings of the Snowmass 95 Summer Study on Particle and Nuclear Astrophysics, edited by E. W. Kolb and R. Peccei (World Scientific, Singapore, 1995).

[11] A. Ori and K. S. Thorne (in preparation).

[12] D. Lai and A. G. Wiseman, Phys. Rev. D 54, 3958 (1996).

[13] S. A. Teukolsky and W. H. Press, Astrophys. J. 193, 443 (1974).

[14] S. Chandrasekhar and S. L. Detweiler, Proc. R. Soc. London A344, 441 (1975).

[15] F. Echeverria, Phys. Rev. D 40, 3194 (1989).

[16] L. S. Finn, Phys. Rev. D 46, 5236 (1992). See also F. Echeverria, Ph.D. thesis, California Institute of Technology, 1993, addendum to Chap. 2. This is an addendum to Ref. [15] which shows that the methods used to calculate measurement errors in Refs. [15] and [16] are equivalent.

[17] G. D. Quinlan and S. L. Shapiro, Astrophys. J. 343, 725 (1989) (especially Fig. 12); 356, 483 (1990).

[18] S. Sigurdsson and L. Hernquist, Nature (London) 364, 423 (1993).

[19] E. S. Phinney, Astrophys. J. 380, L17 (1991).

[20] R. Narayan, T. Piran, and A. Shemi, Astrophys. J. 379, L17 (1991).

[21] E. P. J. Van den Heuvel and D. R. Lorimer, Mon. Not. R. Astron. Soc. 283, L37 (1996).

[22] A. V. Tutukov and L. R. Yungelson, Mon. Not. R. Astron. Soc. 260, 675 (1993).

[23] H. Yamaoka, T. Shigeyama, and K. Nomoto, Astron. Astrophys. 267, 433 (1993).

[24] V. M. Lipunov, K. A. Postnov, and M. E. Prokhorov, New Astron. 2, 43 (1997); see also V. M. Lipunov, K. A. Postnov, and M. E. Prokhorov, Astron. Lett. 24, 492 (1997). 
[25] S. F. P. Zwart and L. R. Yungelson, "Formation and evolution of binary neutron stars," Astron. Astrophys. (to be published); astro-ph/9710347.

[26] It is also conceivable that the $\mathrm{BBH}$ coalescence rates could be higher than these theoretical estimates: high mass stars may have been much more numerous among population III stars due to the lower metallicity at high redshift, so there could be a large population of BBHs that are remnants of population III stars living in galactic halos.

[27] Although there have been suggestions that the globular cluster M15 harbors a black hole of mass $\sim 10^{3} M_{\odot}[28]$, theoretical modeling combined with recent HST observations neither confirms nor rules out this possibility [29].

[28] K. A. Postnov, M. E. Prokhorov, and N. I. Shakura, in High Energy Astrophysics: American and Soviet Perspectives, Proceedings from the U.S.-USSR Workshop on High-Energy Astrophysics, 1989, edited by W. H. G. Lewin, G. W. Clark, R. A. Sunyaev, K. K. Trivers, and D. M. Abramson (National Academy Press, Washington, D.C., 1991), pp. 316-321.

[29] J. D. Dull et al., Astrophys. J. 481, 267 (1997); K. Gebhardt et al., Astron. J. 113, 1026 (1997).

[30] M. Begelman and M. Rees, Gravity's Fatal Attraction: Black Holes in the Universe (Freeman, Basingstoke, England, 1995), and references therein.

[31] R. D. Blandford, in Active Galactic Nuclei: Saas-Fee Adv Course 20, edited by T. Courvoisier and M. Mayor (SpringerVerlag, Berlin, 1990); M. J. Rees, Science 247, 817 (1990;).

[32] D. Hils and P. L. Bender, Astrophys. J. 445, L7 (1995).

[33] E. Poisson, Phys. Rev. D 54, 5939 (1996).

[34] M. C. Begelman, R. D. Blandford, and M. J. Rees, Nature (London) 287, 307 (1980).

[35] H. D. Wahlquist, in "NASA, Relativistic Gravitational Experiments in Space"' Report No. SEE N90-19940 12-90, 1989, pp. 14-17.

[36] M. G. Haehnelt, Mon. Not. R. Astron. Soc. 269, 199 (1994).

[37] N. Roos, J. S. Kaastra, and C. A. Hummel, Astrophys. J. 409, 130 (1993).

[38] A. Sillanpaa et al., Astrophys. J. 325, 628 (1988).

[39] C. M. Gaskell, Astrophys. J. 464, L107 (1996); in Jets from Stars and Galaxies, edited by W. Kundt (Springer, Berlin, 1996), pp. 165-196.

[40] B. F. Schutz, in The Detection of Gravitational Radiation, edited by D. Blair (Cambridge University Press, Cambridge, England, 1989).

[41] L. Blanchet, Phys. Rev. D 54, 1417 (1996).

[42] L. Blanchet (private communication).

[43] By post- $n$-Newtonian order, we mean $O\left[(v / c)^{2 n}\right]$ in secular terms and $O\left[(v / c)^{5+2 n}\right]$ in dissipative, radiation reaction terms $(2 n$ orders beyond the leading quadrupole radiation reaction term). This is the standard counting method for postNewtonian waveforms.

[44] C. Cutler, T. A. Apostolatos, L. Bildsten, L. S. Finn, É. E. Flanagan, D. Kennefick, D. M. Marković, A. Ori, E. Poisson, G. J. Sussman, and K. S. Thorne, Phys. Rev. Lett. 70, 2984 (1993).

[45] E. Poisson, Phys. Rev. D 52, 5719 (1995).

[46] R. Balasubramanian and S. V. Dhurandhar, Phys. Rev. D 50, 6080 (1994).

[47] C. Cutler and É. E. Flanagan (in preparation).

[48] Families of template waveforms optimized for signal searches are in the early stages of development; see B. S.
Sathyaprakash, Phys. Rev. D 50, R7111 (1994); B. S. Sathyaprakash and S. V. Dhurandhar, ibid. 44, 3819 (1991); 49, 1707 (1994); A. Królak, K. D. Kokkotas, and G. Schäfer, in Proceedings of the 17th Texas Symposium on Relativistic Astrophysics [Ann. (N.Y.) Acad. Sci. 793, 493 (1995)]; T. A. Apostolatos, Phys. Rev. D 52, 605 (1995).

[49] K. S. Thorne, presentated at the Intermediate Binary Black Hole workshop, Caltech, 1996. The location of the postNewtonian breakdown can be seen from Fig. 1 of Ref. [45].

[50] A workshop focusing on this issue was held at Caltech in 1996.

[51] Even if such methods are not found, post-Newtonian templates should be adequate for searching for inspiral waves even in the IBBH regime; IBBH templates will likely be needed only as a foundation for extracting the waves' information. We also note that the method of Padé approximants has recently been found to improve significantly the convergence of the post-Newtonian series [52]; the implications of this for the IBBH regime are not yet clear.

[52] T. Damour, B. Iyer, and B. S. Sathyaprakash, Phys. Rev. D 57, 885 (1998).

[53] One can convince oneself of the likelihood of this cleanliness by considering various possible sources of perturbations to the gravitational-wave signal. For solar mass coalescences, the most likely perturbing effect would be due to accretion disks; most likely they will be long gone before the black holes merge. One case where a possible perturbing effect on the gravitational-wave signal may be observable is the stochastic influence of a surrounding stellar cluster on the orbits of members of a SMBH binary during the early stages of the inspiral. While strictly speaking this could make our assumption of the applicability of Wiener optimal filtering invalid for some SMBH inspirals, such inapplicability is not a cause for concern: the SNRs are typically so high anyway that it should be possible to easily detect both the coherent portions of the inspiral radiation and any isolated changes in phase due to close-encounter perturbations.

[54] The Grand Challenge Alliance is an NSF funded collaboration among physicists and computer scientists at eight institutions: University of Texas, Austin; NCSA/University of Illinois; University of North Carolina, Chapel Hill; Cornell University; Syracuse University NPAC; University of Pittsburgh; Northwestern University; and Penn State University. More information can be found at the WWW address http:// jean-luc.ncsa.uiuc.edu/GC/GC.html; see also Ref. [55] for a review.

[55] L. S. Finn, in proceedings of GR14, gr-qc/9603004.

[56] S. Chandrasekhar, The Mathematical Theory of Black Holes (Oxford University Press, New York, 1983), p. 528.

[57] É. É. Flanagan and S. A. Hughes, following paper, Phys. Rev. D 57, 4566 (1998).

[58] C. W. Helstrom, Statistical Theory of Signal Detection (Pergamon, Oxford, 1968).

[59] L. A. Wainstein and V. D. Zubakov, Extraction of Signals from Noise (Prentice-Hall, Englewood Cliffs, NJ, 1962).

[60] C. Cutler and É. E. Flanagan, Phys. Rev. D 49, 2658 (1994).

[61] Our convention for the normalization of the spectral noise density $S_{h}(f)$ is such that

$$
\left\langle n(t)^{2}\right\rangle=\int_{0}^{\infty} S_{h}(f) d f
$$


where $n(t)$ is the detector noise ("one-sided spectral noise density"). This determines the constant 4 appearing in Eq. (2.7). Note that the alternative convention used in Refs. [58, 59] results in a factor of 2 instead of 4 in Eq. (2.7). In Eq. (29) of Ref. [1], the spectral noise density that is used is the one-sided spectral noise density (as in this paper), and thus the overall factor in front of the integral should be 4, not 2 .

[62] S. L. Shapiro and S. A. Teukolsky, Black Holes, White Dwarfs, and Neutron Stars: The Physics of Compact Objects (Wiley, New York, 1983).

[63] For instance, if the post-Newtonian expansion is taken to order $3 / 2$, we would find in the equal mass case (see, e.g., Ref. [64])

$$
\frac{d E}{d f}=\frac{1}{12} \pi^{2 / 3} M^{5 / 3} f^{-1 / 3}\left[1-\frac{1}{6}\left(9+\frac{1}{4}\right)(\pi M f)^{2 / 3}\right] .
$$

The correction to the Newtonian formula is $\sim 0.24$ at $f$ $=f_{\text {merge }}$; when taken to higher order, there will be additional corrections. For the purposes of our discussion, these corrections are not important-an estimate of the SNR that is accurate to a few tens of a percent is adequate. Also, the loss in SNR due the phase lag between the approximate, postNewtonian templates and the true, general-relativistic signal will be small [48].

[64] C. M. Will, Relativistic Cosmology, edited by M. Sasaki (Universal Academy Press, Tokyo, 1994), pp. 83-98.

[65] This is the value predicted by the numerical initial data sets of Ref. [9] at $f=f_{\text {merge }}=0.02 / M$; see also the arguments concerning angular momentum in Sec. III B.

[66] S. R. Brandt and E. Seidel, Phys. Rev. D 52, 870 (1995); the run labeled $\mathrm{r} 3$ of a distorted Kerr black hole with $a \sim 0.35$ yields an energy radiated in ringdown waves of $\sim 3 \%$ (from Table IV and also from Fig. 18 which shows that most of the radiated energy can be ascribed to the ringdown waves).

[67] L. L. Smarr, Ph.D. thesis, University of Texas at Austin, 1975.

[68] S. Detweiler, in Sources of Gravitational Radiation, edited by L. L. Smarr (Cambridge University Press, Cambridge, England, 1979).

[69] See, e.g., J. Baker, A. Abrahams, P. Anninos, S. Brandt, R. Price, J. Pullin, and E. Seidel, Phys. Rev. D 55, 829 (1997).

[70] When we say that a source is visible out to a distance $D$, we mean that the rate of detection of events is the roughly the same as the rate of occurrence of events within a sphere of radius $D$. Note that some events within this sphere will be missed, and some outside this sphere will be detected, due to the beaming and orientation effects discussed in Sec. II C. Rare, optimally oriented sources will be visible out to several times $D$.

[71] It would be quite simple to improve on this filter. For example, one could divide it by the noise spectral density in order to weight the filter according to the instrumental noise characteristics, i.e., pre-whiten the data stream. The main result of our analysis, the formula (2.12), would also approximately apply to such an improved filtering method.

[72] Note that we distinguish between the number of statistically independent waveform shapes $\mathcal{N}_{\text {shapes }}$ in the manifold of waveforms that one is trying to detect using the matched filtering process and the actual number of templates $\mathcal{N}_{\text {templates }}$ used in the computations. The number $\mathcal{N}_{\text {shapes }}$ is the volume of the subset of the manifold of signals with $\mathrm{SNR} \leqslant \rho$, in the natural metric induced by the statistical properties of the noise; thus $\mathcal{N}_{\text {shapes }}$ depends on $\rho$. The number $\mathcal{N}_{\text {templates }}$ is defined in Sec. VI A and is independent of $\rho$. In general $\mathcal{N}_{\text {templates }}<\mathcal{N}_{\text {shapes }}$ since the optimum computational method will have less than one template per statistically independent waveform shape. For the purposes of calculating SNR thresholds, $\ln \left(\mathcal{N}_{\text {templates }}\right) \approx \ln \left(\mathcal{N}_{\text {shapes }}\right)$ to a good approximation.

[73] É. É. Flanagan (in preparation).

[74] P. R. Brady, T. Creighton, C. Cutler, and B. F. Schutz, Phys. Rev. D 57, 2101 (1998).

[75] D. Marković, Phys. Rev. D 48, 4738 (1993); E. W. Kolb and M. S. Turner, The Early Universe (Addison-Wesley, Redwood City, CA, 1990), p. 44.

[76] During the inspiral phase, the time scale over which the frequency of the waves changes is much longer than the instantaneous period, so it makes sense to talk about the frequency of the waves at a certain time. In particular, we can (roughly) talk either about the time or the frequency at which the inspiral ends - the notion of instantaneous frequency is just beginning to break down then.

[77] L. L. Smarr, in Sources of Gravitational Radiation, edited by L. L. Smarr (Cambridge University Press, Cambridge, England, 1979).

[78] P. Anninos, D. Hobill, E. Seidel, L. Smarr, and W.-M. Suen, Phys. Rev. Lett. 71, 2851 (1993); Phys. Rev. D 52, 2044 (1995).

[79] S. L. Detweiler, Proc. R. Soc. London A352, 381 (1977).

[80] D. Christodoulou, Phys. Rev. Lett. 67, 1486 (1991); K. S. Thorne, Phys. Rev. D 45, 520 (1992); A. G. Wiseman and C. M. Will, ibid. 44, R2945 (1991).

[81] D. Kennefick, Phys. Rev. D 50, 3587 (1994).

[82] Since $\epsilon$ is a scalar quantity, it can only depend on scalar products of $\mathbf{S}_{1}, \mathbf{S}_{2}$ and $\hat{\mathbf{L}}$ and not on these vectorial quantities themselves. Therefore the number of independent variables that $\epsilon$ depends on is 6 .

[83] J. K. Blackburn and S. Detweiler, Phys. Rev. D 46, 2318 (1992), the geometry at which $S=7.5 m_{1}$ in their notation.

[84] D. M. Eardley, in Gravitational Radiation, edited by N. Deruelle and T. Piran (North-Holland, Amsterdam, 1983).

[85] See, for example, K. S. Thorne, Rev. Mod. Phys. 52, 299 (1980), Eqs. $\left(4.16^{\prime}\right)$ and $\left(4.23^{\prime}\right)$.

[86] The inequality $\left|\mathbf{S}_{\text {final }}\right| \leqslant M^{2}$ used in deriving Eq. (3.9) should really be $\left|\mathbf{S}_{\text {final }}\right| \leqslant\left(M-E_{\text {rad }}\right)^{2}$, since it is the mass of the final black hole and not the initial total mass of the system that is relevant. An argument parallel to that leading to Eq. (3.11) then yields the inequality $\epsilon_{\mathrm{rad}} \geq\left[1.4-\left(1-\epsilon_{\mathrm{rad}}\right)^{2}\right] / 4$ or $\epsilon_{\mathrm{rad}}$ $\gtrsim 0.18$, where $\epsilon_{\mathrm{rad}}=E_{\mathrm{rad}} / M$. Hence, even if only $\sim 50 \%$ of the emitted radiation is quadrupolar, the estimate $\epsilon_{\text {rad }} \sim 0.1$ used in Sec. III B is probably still fairly realistic.

[87] D. M. Eardley and E. W. Hirschmann, "Comment on 'Instabilities in Close Neutron Star Binaries,',' ITP Santa Barbara Report No. NSF-ITP-95-165, gr-qc/9601019.

[88] C. W. Misner et al., Gravitation (Freeman, New York, 1973), Sec. 36.6.

[89] B. J. Owen, Phys. Rev. D 53, 6749 (1996).

[90] E. W. Leaver, Proc. R. Soc. London A402, 285 (1985). Leaver's data on the $l=m=2$ mode, which was used by Echeverria, is unpublished.

[91] K. S. Thorne, Astrophys. J. 191, 507 (1974).

[92] Actually the QNR metric perturbation will vanish at the event horizon; one could instead use the boundary of the ergoregion. Also, the ratio $\mathcal{R}$ of the circumferences will be gauge 
dependent. However, if we expand $\mathcal{R}$ as $\mathcal{R}=1+\alpha \mathcal{A}$ $+O\left(\mathcal{A}^{2}\right)$, then the coefficient $\alpha$ will be gauge invariant and we could take $\mathcal{A}_{2}=1 / \alpha$.

[93] More specifically, an ellipsoidal solid body of mass $M$ of uniform density whose axes are given by $l^{2}, l^{2}[1$ $\pm(3 / 5) \sin (\omega t)]$ produces a luminosity $L=9 M^{2} l^{4} \omega^{6} / 5^{5}$; comparing this to the luminosity $\mathcal{A}^{2} M^{2} \omega_{\mathrm{qnr}}^{2} /(16 \pi)$ for the waveform (3.15) at $t=0$, identifying $\omega=\omega_{\mathrm{qnr}}=2 \pi 0.13 / M$ and taking $l=r_{+}=M+\sqrt{M^{2}-a^{2}}$ for $a=0.97 M$ say yields $\mathcal{A}$ $=0.4$. This crude estimation method clearly does not take into account the potential barrier through which the radiation must propagate (among other things). We imagine a more precise calculation will find a somewhat smaller value of $\mathcal{A}$.

[94] This error can be seen by inserting the parameter values $m$ $=1000 \mathrm{~kg}, f_{0}=1 \mathrm{~Hz}$, and $Q_{0}=10^{9}$ given in Ref. [3] into the standard equation for suspension thermal noise due to viscous damping, as given in, e.g., Eq. (4.3) of Ref. [95]. The resulting noise level is 3 times larger than the noise level shown in Fig. 7 of Ref. [3] for $10 \mathrm{~Hz} \lesssim f \leqq 70 \mathrm{~Hz}$. This error was not made in the corresponding Fig. V.4 of the 1989 LIGO proposal [96] [K. S. Thorne (private communication)].

[95] L. S. Finn and D. F. Chernoff, Phys. Rev. D 47, 2198 (1993).

[96] R. E. Vogt et al., "Proposal to the National Science Foundation for the Construction, Operation, and Supporting Research and Development of a Laser Interferometer Gravitational-Wave Observatory," California Institute of Technology, 1989 (unpublished).

[97] The $h_{\mathrm{rms}}(f)=$ const part of the noise curve happens to have the same frequency dependence that the test masses' thermal noise would have if this noise were due to structural damping [98]. This behavior is accidental: the $h_{\mathrm{rms}}(f)=$ const piece of the spectrum is used here merely to more closely approximate the smooth transition between the suspension thermal noise and laser shot noise parts of the spectrum.

[98] A. Gillespie and F. Raab, Phys. Lett. A 190, 213 (1994).

[99] C. Cutler, "Angular Resolution of the LISA Gravitational Wave Detector,' gr-qc/9703068 [Phys. Rev. D (to be published)].

[100] See Eq. (32) of Ref. [1]. Note also that our $h_{n}(f)$ is similar to the quantity $h_{3 / \mathrm{yr}}$ defined in Ref. [1], which is the same as the quantity denoted $h_{\mathrm{SB}}(f)$ in Refs. [3,10]. These quantities differ from $h_{n}(f)$ by the factor $\sqrt{\ln \mathcal{N}} \sim 5$, where $\mathcal{N}$ is the number of independent starting times per third of a year that must be tried in the matched filtering search process; see Eq. (34) of Ref. [1]. We omit this factor in our definition of $h_{n}$ since in paper II [57] we will be discussing SNRs in situations when the waves have already been detected and where the factor $\sqrt{\ln \mathcal{N}}$ is not relevant. The factor instead appears in our SNR thresholds discussed in Sec. VI. Note also that our signal-to-noise ratios differ from those in Refs. $[3,10]$ by an overall factor of $\sqrt{3 / 2}$, as discussed after Eq. (2.30) above.

[101] We obtained the metric (6.4) in the following way: (i) We took the large $Q$ limit of the matrix $\mathcal{C}_{i j}^{\prime-1}$ of Ref. [16], which was calculated assuming white noise. (ii) We inverted this matrix, took a $2 \times 2$ sub-block and re-inverted, thus eliminating (effectively integrating out) the variables $V$ and $T$. This procedure for eliminating the time-of-arrival variable $T$ is equivalent to the procedure embodied in Eq. (2.28) of Ref. [89]. (iii) We divided by 2 in order to accord with the definition (2.12) of Ref. [89].

[102] This is Eq. (2.9) with $\ln \left(\mathcal{N}_{\text {shapes }}\right)$ replaced by $\ln \left(\mathcal{N}_{\text {templates }}\right): \mathrm{cf}$. Ref. [72] above.

[103] In the situation where merger templates are available, the optimum search method would in fact be a combination of inspiral, merger and ringdown searches, in which the entire inspiral-merger-ringdown signal as a whole is searched for using matched filtering.

[104] Here we are assuming that we have a fair idea of the range of durations and bandwidths of merger waves from representative supercomputer simulations. Without such information, a more appropriate, conservative value for $\mathcal{N}_{\text {bins }}$ might be $\sim 300$. This would increase $\mathcal{R}$ from 3.5 to $\sim 10$, corresponding to a reduction in the event rate of $\sim 3$. Thus, information from the simulations will be important for detecting the waves.

[105] The quantity $\mathcal{N}_{\text {shapes,max }}$ is also approximately the total of waveform shapes of duration less than or equal to $T$, with bandwidth $\Delta f$ and with $\mathrm{SNR} \leqslant \rho$ which are distinguishable in the interferometer noise [57].

[106] To see that angle-averaging the approximate formula (A3) yields the formula (B13) for the ringdown SNR, corresponding to the approximate delta function energy spectrum (3.19), proceed as follows. Starting from Eq. (A14), average over the angles $\theta, \varphi, \psi, \iota$ and $\beta$ using Eqs. (2.34) and (3.16). This yields

$$
\left\langle h_{0}(\theta, \varphi, \psi, \iota, \beta)^{2}\right\rangle=\frac{1}{10 \pi} \frac{\mathcal{A}^{2} M^{2}}{r^{2}} .
$$

Substitute this into Eq. (A3), make the replacement $S_{h}$ $\rightarrow S_{h}\left(f_{\text {qnr }}\right)$, and make the appropriate substitutions to account for cosmological effects as explained in Sec. II C to obtain Eq. (B13).

[107] The small $z$, equal-mass limit of Eq. (B14) agrees with the large $Q$ limit of Eq. (4.7) of Ref. [16] when that equation is multiplied by a correction factor of 2 . 